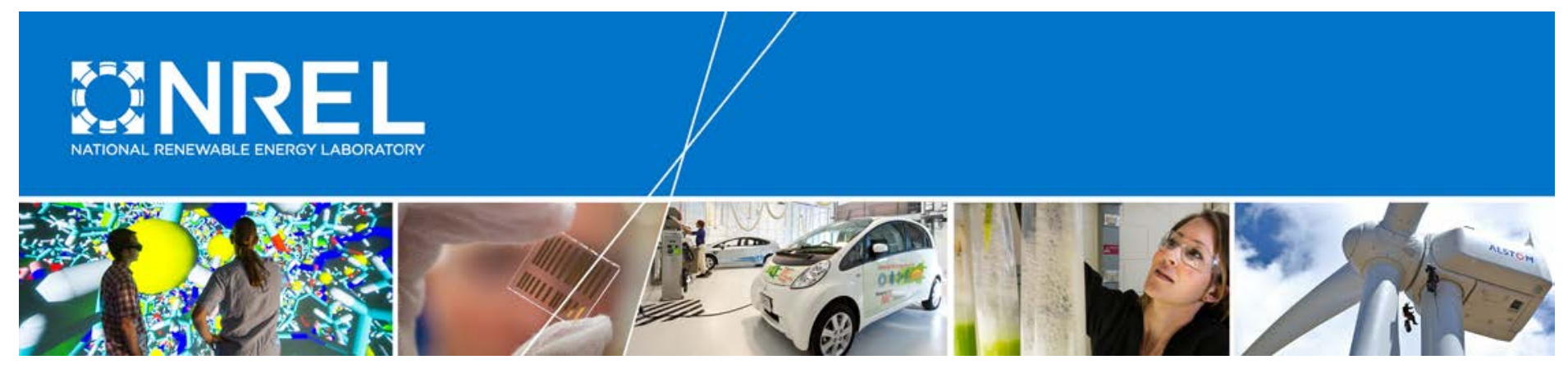

\title{
EPA GHG Certification of Medium- and Heavy-Duty Vehicles: Development of Road Grade Profiles Representative of US Controlled Access Highways
}

Eric Wood, Adam Duran, Evan Burton, Jeffrey Gonder, and Kenneth Kelly

Produced under direction of the U.S. Environmental Protection Agency (EPA) by the National Renewable Energy Laboratory (NREL) under Interagency Agreement IAG-14-1954 and Task No WFGY.1000.

NREL is a national laboratory of the U.S. Department of Energy Office of Energy Efficiency \& Renewable Energy Operated by the Alliance for Sustainable Energy, LLC

This report is available at no cost from the National Renewable Energy Laboratory (NREL) at www.nrel.gov/publications.

Strategic Partnership Project Report NREL/TP-5400-63853

May 2015

Contract No. DE-AC36-08GO28308 


\section{EPA GHG Certification of Medium- and Heavy-Duty Vehicles: Development of Road Grade Profiles Representative of US Controlled Access Highways}

Eric Wood, Adam Duran, Evan Burton, Jeffrey Gonder, and Kenneth Kelly

Prepared under Task No. WGFY.1000

NREL is a national laboratory of the U.S. Department of Energy Office of Energy Efficiency \& Renewable Energy Operated by the Alliance for Sustainable Energy, LLC

This report is available at no cost from the National Renewable Energy Laboratory (NREL) at www.nrel.gov/publications.

National Renewable Energy Laboratory 15013 Denver West Parkway Golden, CO 80401

303-275-3000 • www.nrel.gov
Strategic Partnership Project Report

NREL/TP-5400-63853

May 2015

Contract No. DE-AC36-08G028308 


\section{NOTICE}

This manuscript has been authored by employees of the Alliance for Sustainable Energy, LLC ("Alliance") under Contract No. DE-AC36-08GO28308 with the U.S. Department of Energy ("DOE”).

This report was prepared as an account of work sponsored by an agency of the United States government. Neither the United States government nor any agency thereof, nor any of their employees, makes any warranty, express or implied, or assumes any legal liability or responsibility for the accuracy, completeness, or usefulness of any information, apparatus, product, or process disclosed, or represents that its use would not infringe privately owned rights. Reference herein to any specific commercial product, process, or service by trade name, trademark, manufacturer, or otherwise does not necessarily constitute or imply its endorsement, recommendation, or favoring by the United States government or any agency thereof. The views and opinions of authors expressed herein do not necessarily state or reflect those of the United States government or any agency thereof. 


\section{List of Acronyms}

\begin{tabular}{ll} 
ADA & Advanced Driving Attributes \\
DEM & Digital Elevation Model \\
DOE & U.S. Department of Energy \\
DRIVE & Drive Cycle Rapid Investigation, Visualization, and \\
& Evaluation tool \\
EPA & U.S. Environmental Protection Agency \\
GHG & greenhouse gas \\
GPS & global positioning system \\
HD & heavy duty \\
HH & half hill \\
Hz & hertz \\
I & Interstate Highway \\
KS & Kolmogorov-Smirnov \\
MARTI & Mobile Autonomous Robotics Technology Initiative \\
MD & medium duty \\
MOVES & Motor Vehicle Emission Simulator \\
mph & miles per hour \\
NED & National Elevation Dataset \\
NHTSA & U.S. National Highway Traffic Safety \\
NREL & Administration \\
RMSE & National Renewable Energy Laboratory \\
SR & root mean square error \\
SwRI & State Route \\
U.S. & Southwest Research Institute \\
USGS & United States \\
VMT & United States Geological Survey \\
& vehicle miles traveled \\
\hline
\end{tabular}




\section{Table of Contents}

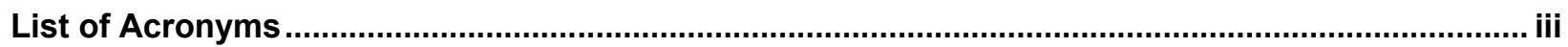

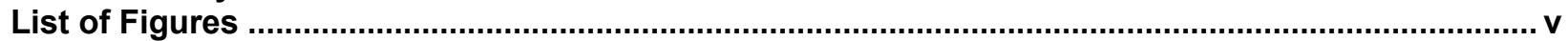

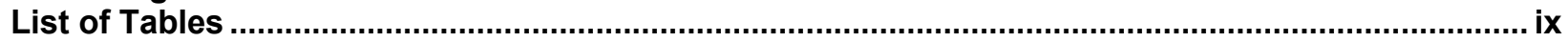

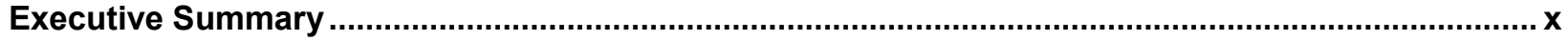

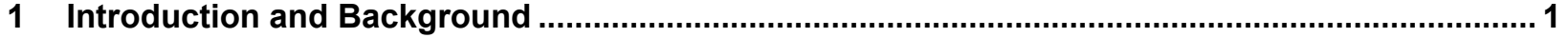

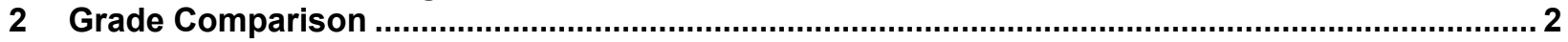

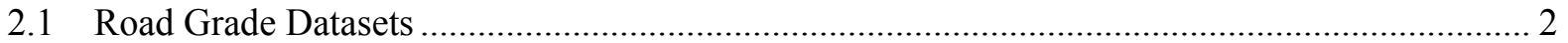

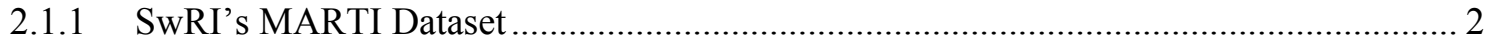

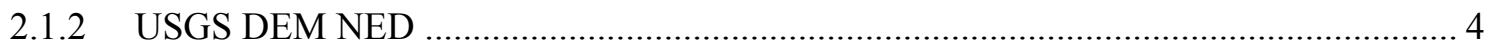

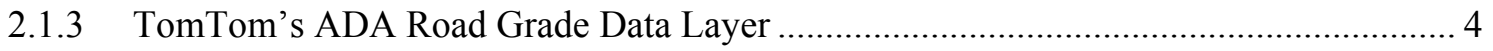

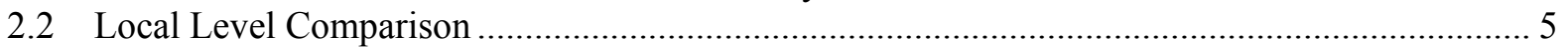

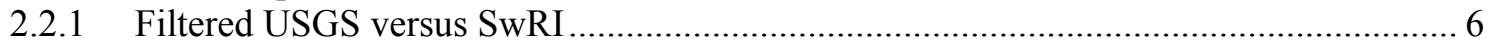

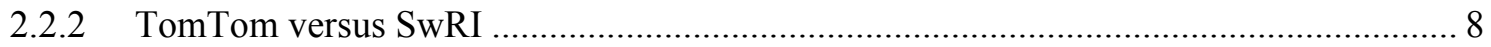

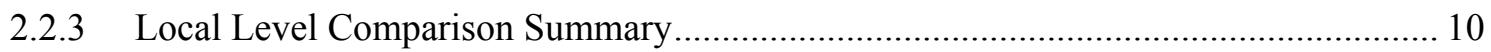

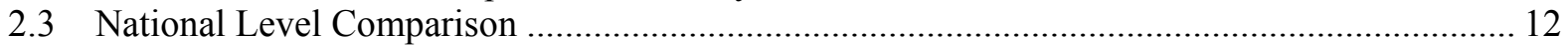

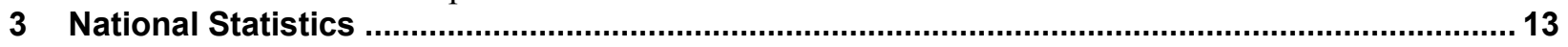

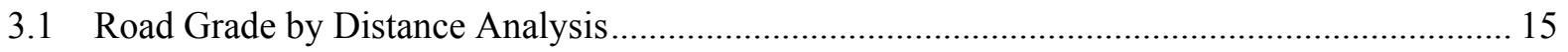

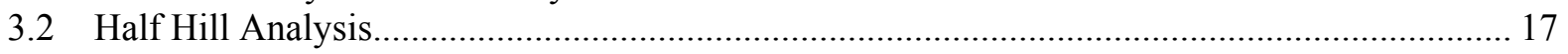

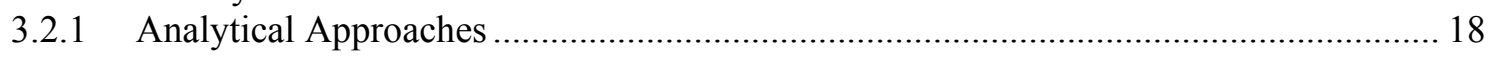

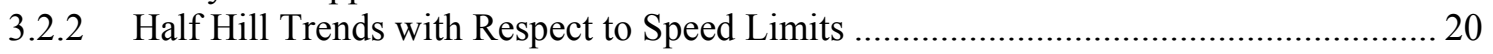

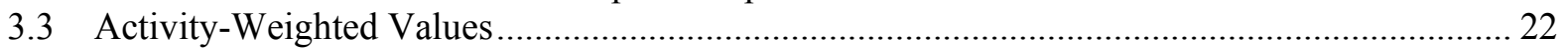

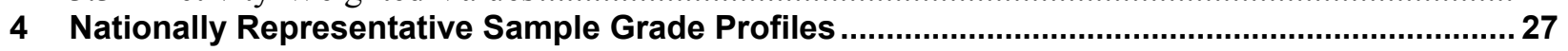

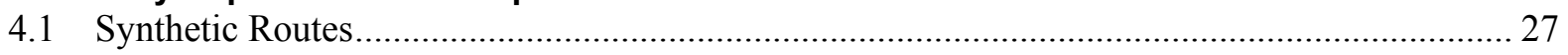

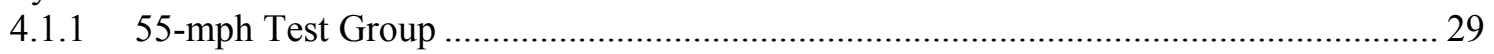

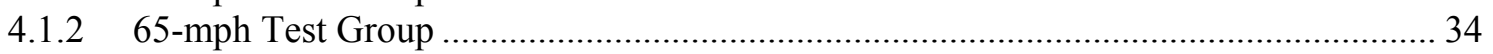

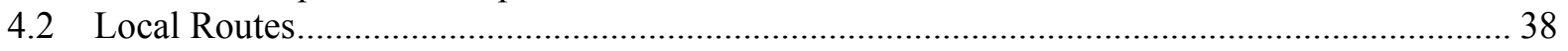

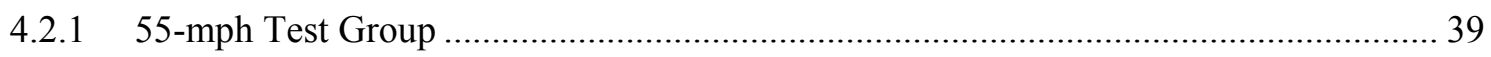

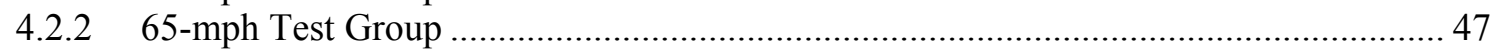

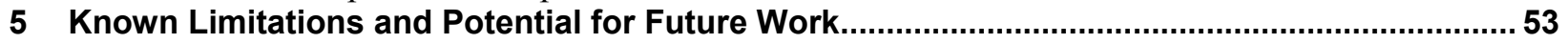

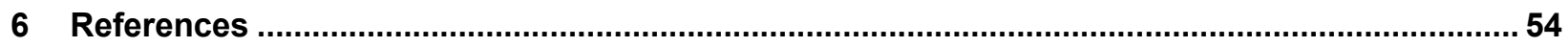

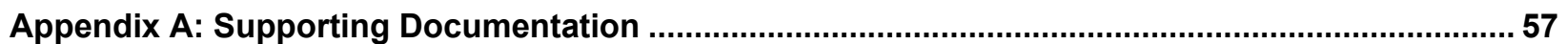

Appendix B: Supplementary Distributions from Half Hill Analysis .................................................. 64 


\section{List of Figures}

Figure 1. Three-hundred-mile loop near San Antonio, Texas, selected for SwRI on-road testing of Class 8 combination truck. The loop was also used for data collection by SwRI MARTI vehicle...... 3

Figure 2. United States elevation map utilizing USGS 1/3 arc-second DEM.......................................... 4

Figure 3. U.S. controlled access highways by TomTom ADA road grade .............................................. 5

Figure 4. USGS elevation map near San Antonio, Texas, overlaid with GPS trace of MARTI vehicle driving from Junction, Texas, to San Antonio, Texas, on I-10 .......................................... 6

Figure 5. SwRI/USGS comparison over the 115-mile stretch of I-10 northwest of San Antonio, Texas. Top: SwRI integrated grade plotted against filtered USGS elevation. Bottom: SwRI grade plotted against filtered, differentiated USGS elevation.

\section{6}

Figure 6. SwRI/USGS comparison over a subset of the 115-mile stretch of I-10 northwest of San Antonio,
Texas. Top: SwRI integrated grade plotted against filtered USGS elevation. Bottom: SwRI grade plotted against filtered, differentiated USGS elevation............................................. 7

Figure 7. Filtered USGS grade scattered against SwRI grade from the 115-mile stretch of I-10 northwest

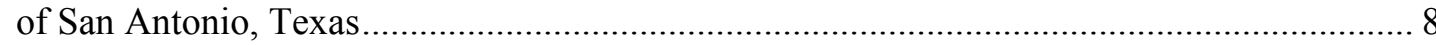

Figure 8. SwRI/TomTom comparison over the 115-mile stretch of I-10 northwest of San Antonio, Texas. Top: SwRI integrated grade plotted against integrated TomTom grade. Bottom: SwRI grade

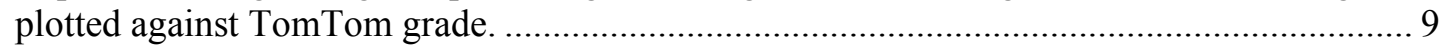

Figure 9. SwRI/TomTom comparison over a subset of the 115-mile stretch of I-10 northwest of San Antonio, Texas. Top: SwRI integrated grade plotted against integrated TomTom grade. Bottom: SwRI grade plotted against TomTom grade.

Figure 10. TomTom grade scattered against SwRI grade from the 115-mile stretch of I-10 northwest of

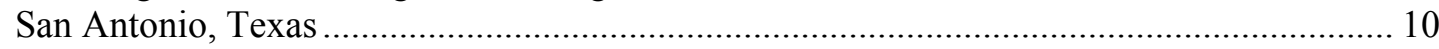

Figure 11. Visual description of the two sampling frequencies used in the comparative analysis: 1) based on available data, and 2) based on linear interpolation at uniform intervals of 10 feet $(0.1$ mile interval is used for illustrative purposes)....

Figure 12. National distributions of road grade from filtered USGS and TomTom databases on the basis

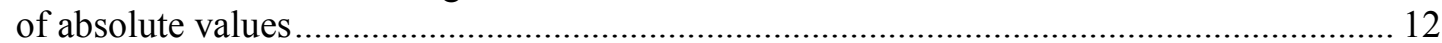

Figure 13. TomTom MultiNet map of U.S. controlled access highways............................................... 13 Figure 14. U.S. controlled access highway map colored by state-defined truck speed limits per TomTom Trucking Logistics speed restrictions data layer....

Figure 15. Mileage breakdown of U.S. controlled access highways by truck speed limit and U.S. Census

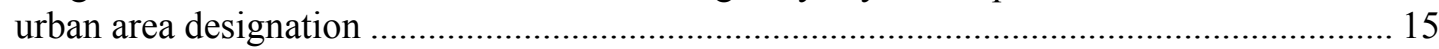

Figure 16. Cumulative distributions of road grade by distance for 1,728 U.S. counties .......................... 16

Figure 17. Raw distance-based cumulative distribution of absolute road grade by truck speed limit........ 17 Figure 18. Example section of controlled access highway broken into 16 half hills (eight climbs and eight

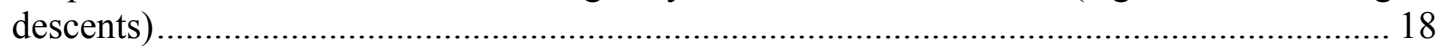

Figure 19. All half hill $(\mathrm{HH})$ distances plotted as frequency-based and distance-based cumulative

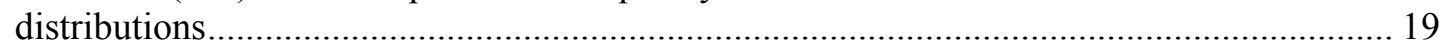

Figure 20. Raw distance-based cumulative distributions of half hill distance by truck speed limit ...........20

Figure 21. Maximum (or minimum) versus average road grade for all measured half hills..................... 21

Figure 22. EPA MOVES VMT database plotted by vehicle type and roadway classification .................. 22

Figure 23. EPA MOVES VMT database for MD/HD vehicles on controlled access roads only ............... 22

Figure 24. EPA MOVES VMT database mapped by county for MD/HD trucks operating on controlled

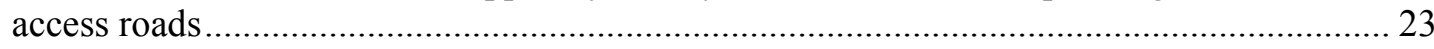

Figure 25. Activity-weighted, distance-based cumulative distributions of absolute road grade by truck speed limit ........................................................................................................... 24

Figure 26. Activity-weighted, distance-based cumulative distributions of half hill distance by truck speed

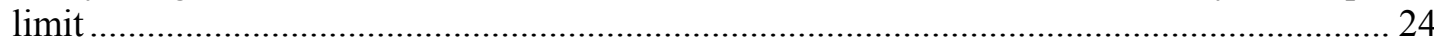


Figure 27. Combined activity-weighted, distance-based cumulative distributions of absolute road grade by

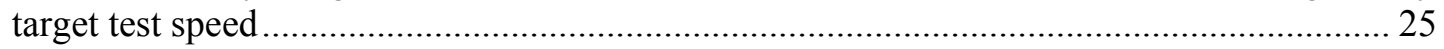

Figure 28. Combined activity-weighted, distance-based cumulative distributions of half hill distance by

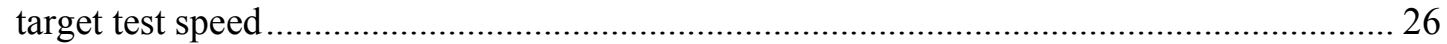

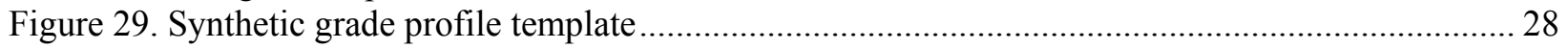

Figure 30. KS statistics for absolute road grade match in the 55-mph test group with respect to target profile distance and maximum iteration count .................................................................... 29

Figure 31. KS statistics for half hill distance match in the 55-mph test group with respect to target profile

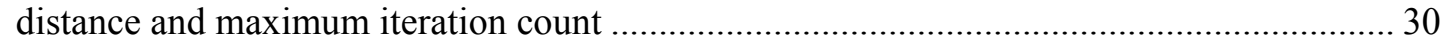

Figure 32. Elevation and road grade profile from sample "150508 55mph 12mi 201210ofle6iter" .........31

Figure 33. Comparison of distance-based cumulative distributions between national activity-weighted dataset and sample profile " $15050855 \mathrm{mph} 12 \mathrm{mi} 201210$ ofle6iter" in terms of absolute road

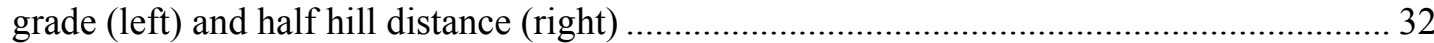

Figure 34. Elevation and road grade profile from sample " $15050855 \mathrm{mph} 12 \mathrm{mi} 727695$ ofle6iter" ......... 32

Figure 35. Comparison of distance-based cumulative distributions between a national activity-weighted dataset and sample profile " $15050855 \mathrm{mph} 12 \mathrm{mi} 727695$ ofle6iter" in terms of absolute road grade (left) and half hill distance (right)

Figure 36. KS statistics for absolute road grade match in the 65-mph test group with respect to target profile distance and maximum iteration count.................................................................. 34

Figure 37. KS statistics for half hill distance match in the 65-mph test group with respect to target profile

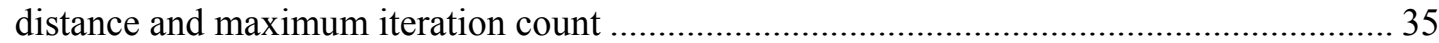

Figure 38. Elevation and road grade profile from sample "150508 65mph 15mi 467684ofle6iter" ......... 36

Figure 39. Comparison of distance-based, cumulative distributions between national activity-weighted dataset and sample profile " $15050865 \mathrm{mph} 15 \mathrm{mi} 467684$ ofle 6 iter" in terms of absolute road

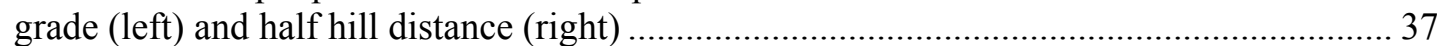

Figure 40. Elevation and road grade profile from sample "150508 65mph 15mi 883894ofle6iter" ........ 37

Figure 41. Comparison of distance-based cumulative distributions between national activity-weighted dataset and sample profile " $15050865 \mathrm{mph} 15 \mathrm{mi} 883894$ ofle6iter" in terms of absolute road

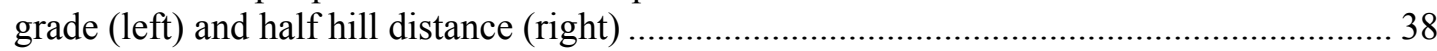

Figure 42. Example 13-mile stretch of roadway segmented into 16 half hills and grouped into seven

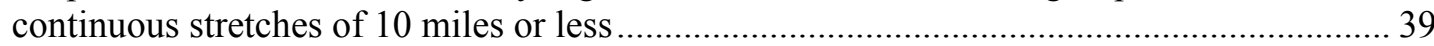

Figure 43. Local route KS statistics for the distribution of absolute road grade versus round-trip profile

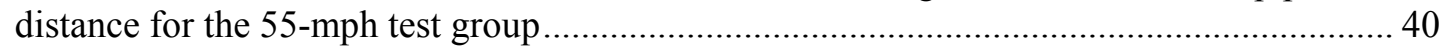

Figure 44. Local route KS statistics for the distribution of half hill distance versus round-trip profile

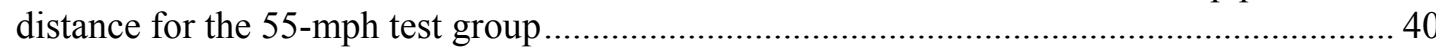

Figure 45. Local route from I-20 east of Abilene, Texas, statistically similar to national activity-weighted

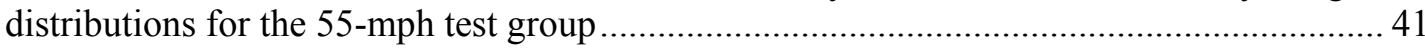

Figure 46. Local route from I-70 east of Glenwood Springs, Colorado, statistically similar to national activity-weighted distributions for the 55-mph test group ................................................... 42

Figure 47. Local route from Kentucky SR-9001 south of Louisville, Kentucky, statistically similar to national activity-weighted distributions for the 55-mph test group ..................................... 42

Figure 48. Local route from I-20 east of Abilene, Texas, statistically similar to national activity-weighted

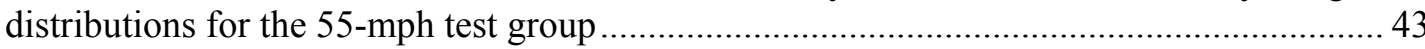

Figure 49. Local route from I-70 east of Glenwood Springs, Colorado, statistically similar to national activity-weighted distributions for the 55-mph test group .................................................. 43

Figure 50. Local route from Kentucky SR-9001 south of Louisville, Kentucky, statistically similar to national activity-weighted distributions for the 55-mph test group .................................... 44

Figure 51. Comparison of distance-based cumulative distributions between national activity-weighted dataset and local profile from I-20 east of Abilene, Texas, in terms of absolute road grade (left) and half hill distance (right) 
Figure 52. Comparison of distance-based cumulative distributions between national activity-weighted dataset and local profile from I-70 east of Glenwood Springs, Colorado, in terms of absolute road grade (left) and half hill distance (right)....

Figure 53. Comparison of distance-based cumulative distributions between national activity-weighted dataset and local profile from Kentucky SR-9001 south of Louisville, Kentucky, in terms of absolute road grade (left) and half hill distance (right).

Figure 54. Local route KS statistics for the distribution of absolute road grade versus round-trip profile distance for the 65-mph test group.

Figure 55. Local route KS statistics for the distribution of half hill distance versus round-trip profile distance for the 65-mph test group......

Figure 56. Local route from I-10 west of San Antonio, Texas, statistically similar to national activityweighted distributions for the 65 -mph test group .....

Figure 57. Local route from I-40 west of Gallup, New Mexico, statistically similar to national activityweighted distributions for the 65-mph test group

Figure 58. Local route from I-10 west of San Antonio, Texas, statistically similar to national activity-

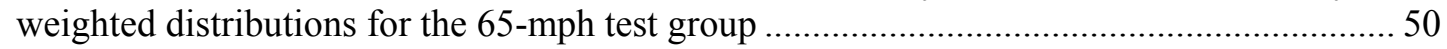

Figure 59. Local route from I-40 west of Gallup, New Mexico, statistically similar to national activity-

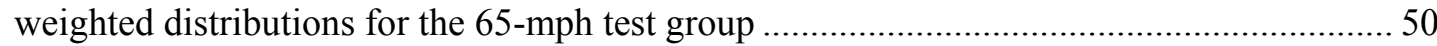

Figure 60. Comparison of distance-based cumulative distributions between national activity-weighted dataset and local profile from I-10 west of San Antonio, Texas, in terms of absolute road grade (left) and half hill distance (right) .....

Figure 61. Comparison of distance-based cumulative distributions between national activity-weighted dataset and local profile from I-40 west of Gallup, New Mexico, in terms of absolute road grade (left) and half hill distance (right).....

Figure 62. Vehicle fueling rate, road grade, vehicle speed, and vehicle to engine speed ratio for a 10minute section of SwRI on-road testing of a class 8 combination truck ................................ 53

Figure A1. Three generalized categories of elevation profiles ........................................................ 57

Figure A2. Elevation profile, grade profile, and grade histogram for example 2.8-mile stretch of road.... 58

Figure A3. Elevation profile, grade profile, and grade histogram for round trip on example 2.8-mile

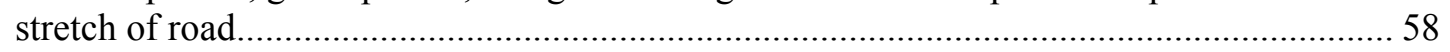

Figure A4. Elevation profile, absolute grade profile, and absolute grade histogram for example 2.8-mile

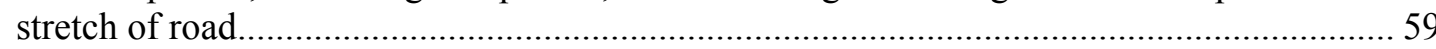

Figure A5. Supremum measurement example for national and synthetic cumulative distributions of absolute road grade ....

Figure A6. Scatter of activity-based versus distance-based county weights for the sample of 1,728 U.S. counties with controlled access highways..

Figure A7. Scatter of average absolute road grade versus the difference between activity- and distancebased weights for the sample of 1,728 U.S. counties with controlled access highways ........ 62

Figure A8. Raw distance-based cumulative distributions of grade by distance and average half hill grade for the 55-mph test group....

Figure B1. Raw frequency-based cumulative distributions of half hill distance by truck speed limit........ 64

Figure B2. Raw frequency-based cumulative distributions of half hill average grade by truck speed limit65

Figure B3. Raw distance-based cumulative distributions of half hill average grade by truck speed limit . 65

Figure B4. Activity-weighted distance-based cumulative distributions of half hill average grade by truck speed limit

Figure B5. Raw frequency-based cumulative distributions of half hill average grade by half hill distance66

Figure B6. Raw frequency-based cumulative distributions of half hill grade ratio (maximum to average half hill grade) by truck speed limit

Figure B7. Raw distance-based cumulative distributions of half hill grade ratio (maximum to average half hill grade) by truck speed limit 
Figure B8. Activity-weighted distance-based cumulative distributions of half hill grade ratio (maximum

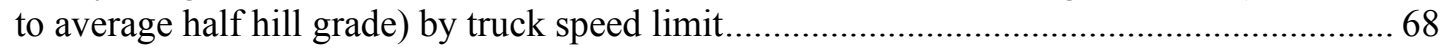

Figure B9. Raw frequency-based cumulative distributions of half hill grade ratio (maximum to average half hill grade) by half hill distance 


\section{List of Tables}

Table 1. Numerical comparison of national datasets (USGS, TomTom) relative to SwRI data from 115mile stretch of I-10 outside San Antonio, Texas ............................................................. 11

Table 2. Summary of three local routes statistically similar to national activity-weighted distributions for

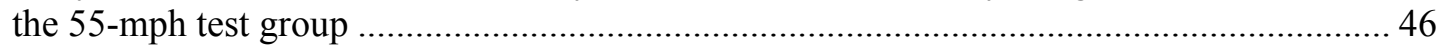

Table 3. Summary of two local routes statistically similar to national activity-weighted distributions for

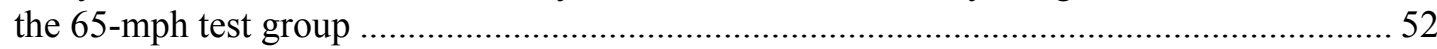




\section{Executive Summary}

In collaboration with the U.S. Environmental Protection Agency and the U.S. Department of Energy, the National Renewable Energy Laboratory has conducted a national analysis of road grade characteristics experienced by U.S. medium- and heavy-duty trucks on controlled access highways. These characteristics have been developed using TomTom's commercially available street map and road grade database.

This report includes a detailed comparison of the TomTom national road grade database relative to a local road grade dataset generated by Southwest Research Institute and a national elevation dataset publically available from the U.S. Geological Survey. This analysis concluded that the TomTom national road grade database was a suitable source of road grade data for purposes of this study.

Using the TomTom national road grade database, national statistics on road grade and hill distances were generated for the U.S. network of controlled access highways. These statistical distributions were then weighted using data provided by the U.S. Environmental Protection Agency on activity of medium- and heavy-duty trucks on controlled access highways.

The national activity-weighted road grade and hill distance distributions were then used as targets for development of a handful of sample grade profiles potentially to be used in the U.S. Environmental Protection Agency's Greenhouse Gas Emissions Model certification tool as well as in dynamometer testing of medium- and heavy duty vehicles and their powertrains.

Future work in this area may include the development of a highway drive cycle with transient speeds aligned to reflect real-world operation of medium- and heavy-duty vehicles on controlled access highways. Such a drive cycle would consider the correlation between vehicle speed and road grade as observed in on-road data. 


\section{Introduction and Background}

In August of 2011, the U.S. Environmental Protection Agency (EPA) and the National Highway Traffic Safety Administration (NHTSA) adopted a national program to reduce greenhouse gas (GHG) emissions and improve fuel efficiency of medium-duty (MD) and heavy-duty (HD) trucks [1-4]. In February of 2014, President Obama announced efforts to update MD/HD vehicle regulations in Phase 2 of EPA's national GHG program [5-6].

As part of the EPA's proposed Phase 2 rulemaking, the U.S. Department of Energy (DOE) and EPA are partnering to support a targeted project to refine and evaluate appropriate duty cycles for tractor-trailers and vocational vehicles to be used as part of MD/HD vehicle certification procedures for GHG emission standards. The National Renewable Energy Laboratory (NREL) will provide technical leadership for this work utilizing DOE-supported data, tools, and expertise.

NREL's experience with large transportation database projects, including the Transportation Secure Data Center [7] and Fleet DNA [8], demonstrates the prerequisite capabilities for tackling data-intense problems. Additionally, development of data analysis tools including the Drive Cycle Rapid Investigation, Visualization, and Evaluation tool (DRIVE) [9] showcases NREL's ability to distill large volumes of information into statistically representative subsets suitable for testing and evaluation purposes. These skillsets combined with NREL's long-standing efforts in evaluating the on-road performance of conventional and advanced technology MD/HD vehicles for large commercial fleets make it an ideal organization for national analysis of the U.S. controlled access highway network.

In particular, this report documents work done to characterize nationally representative road grade profiles for certification testing and simulation on highway drive cycles. Current EPA highway cruise drive cycles are performed at constant speeds of 55 and 65 miles per hour (mph) with zero road grade. The goal of this project was to develop and identify road grade profiles that are representative of U.S. controlled access highways.

This analysis will:

- Evaluate the accuracy and coverage of multiple road grade datasets

- Generate activity-weighted distributions of road grade and hill length at the national level

- Propose several potential grade profiles for testing and simulation at highway speeds. Additionally, limitations of this work and the potential for continued development of highway drive cycles for EPA certification are discussed. 


\section{Grade Comparison}

Road grade is known to have a significant impact on the fuel efficiency and emissions of lightduty vehicles [10-13]. These impacts are amplified for MD/HD vehicles operating at generally heavier weights than typical light-duty passenger vehicles [14-17]. Until recently, the ability to quantify these impacts has been limited by the lack of a comprehensive national database of high-accuracy road grades.

Three road grade datasets are compared:

- Southwest Research Institute (SwRI) Mobile Autonomous Robotics Technology Initiative (MARTI) measurements near San Antonio, Texas

- United States Geological Survey (USGS) Digital Elevation Model (DEM) of the National Elevation Dataset (NED) (Release: 2009)

- TomTom Advanced Driving Attributes (ADA) road grade data layer (Release: 2014 3Q).

First, each dataset is described. Second, a detailed comparison is made over a 115-mile stretch of Interstate Highway 10 (I-10) northwest of San Antonio, Texas (coincident with data available from SwRI). Last, an aggregate comparison between USGS and TomTom data is conducted.

\subsection{Road Grade Datasets}

\subsubsection{SwRI's MARTI Dataset}

During 2013, EPA contracted with SwRI to study the impact of road grade on HD vehicles. As part of this effort, SwRI conducted on-road and dynamometer testing of a Class 8 combination truck. On-road testing consisted of multiple passes over a 300-mile highway loop in Texas between San Antonio, Uvalde, and Junction (highways US-90, US-83, and I-10) as shown in Figure 1. 


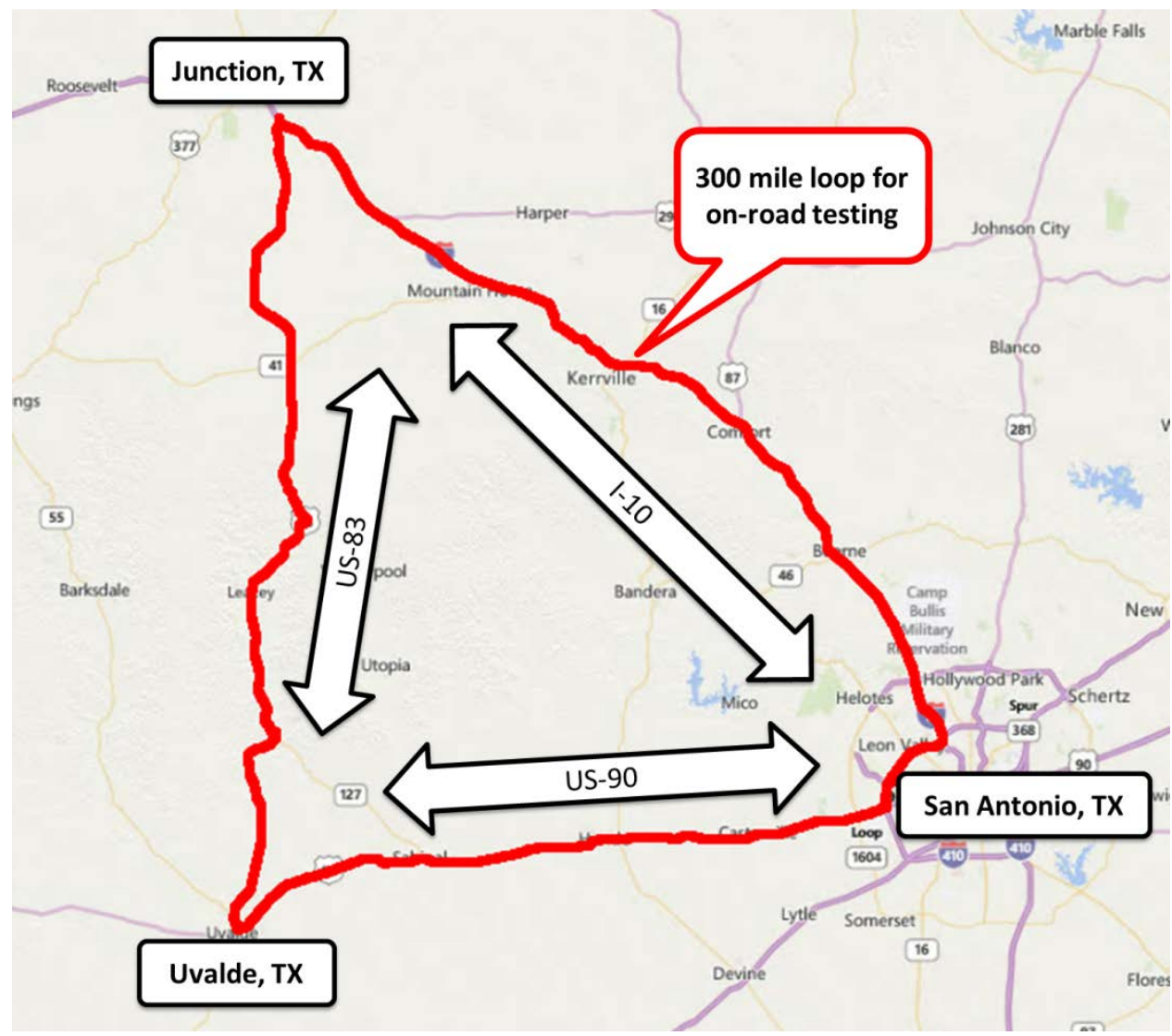

Figure 1. Three-hundred-mile loop near San Antonio, Texas, selected for SwRI on-road testing of Class 8 combination truck. The loop was also used for data collection by SwRI MARTI vehicle.

(Bing Maps credit: @ 2015 Bing, Map Data)

Additionally, SwRI collected on-road data from the same 300-mile loop using its MARTI vehicle (part of SwRI's MARTI Program [18]). The SwRI MARTI vehicle is a light-duty sport utility vehicle equipped with multiple sensing devices, including a high-resolution global positioning system (GPS) and inertial navigation system (three-axis inertial measurement unit with multiple accelerometers and gyroscopes).

Using measurements from the MARTI vehicle, SwRI estimated road grades along the 300-mile highway route. The road grades were spatially aligned with the time series fueling measurements from the Class 8 combination truck on-road data. As expected, a strong correlation between fueling rates and road grade was found. Details of this analysis can be found in SwRI's presentation at the Technical Workshop supporting the need for including the effects of grade in EPA and NHTSA Phase 2 Standards for MD/HD GHG and Fuel Efficiency [4].

This analysis utilizes the MARTI-derived grade estimates from the 115-mile stretch of I-10 in Texas between San Antonio and Junction (the controlled access portion of the original 300-mile route) sampled at a nominal frequency of 100 hertz $(\mathrm{Hz})$. 


\subsubsection{USGS DEM NED}

The USGS makes DEMs publically available as part the NED [19-20]. For this analysis, NREL utilized a 1/3 arc-second DEM, which forms an approximate 10-meter $\times 10$-meter grid across the United States. The $1 / 3$ arc-second DEM is the finest resolution map with complete national coverage currently available from the USGS. Figure 2 shows a graphical representation of the national map. This DEM is a composite of several regional data sources digitally stitched together to form a seamless elevation map of the United States. Regional elevation datasets are generated using a variety of methods, including electronic image correlation, manual profiling, laser illuminated detection and ranging (lidar) digital camera correlation, and other remote sensing techniques. The USGS is continually updating its DEMs and is in the process of populating a NED with complete national coverage at 1-meter resolution as part of the 3D Elevation Program [21].

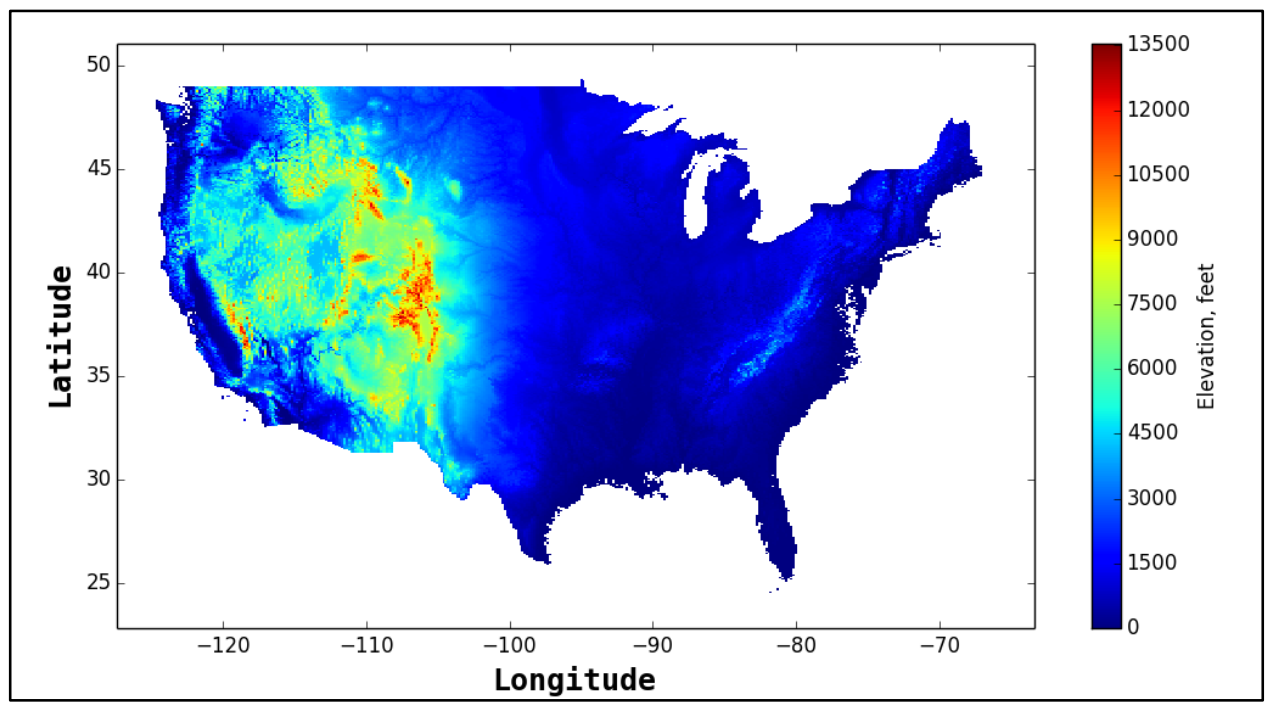

Figure 2. United States elevation map utilizing USGS 1/3 arc-second DEM

As part of NREL's standard routine for processing vehicle GPS data for light-duty and MD/HD vehicle drive cycle databases [7-8], elevations from the USGS DEM are matched to each recorded point in a GPS trace. The raw elevation data are filtered, smoothed, and differentiated to calculate an estimate of road grades corresponding to second-by-second vehicle speed and fuel use data. This process was calibrated in 2014 to a commercially available road-grade database with a root mean square error (RMSE) of $1.48 \%$ road grade. For additional details on this process and calibration of the filtering routine, please refer to [22].

\subsubsection{TomTom's ADA Road Grade Data Layer}

TomTom's MultiNet road network and ADA road grade data layers [23-24] are used as a reference point for national road grade data. TomTom populates its road grade database using a combination of advanced mobile terrestrial sensing and aggregated mobile terrestrial sensing data [25].

Collection of advanced mobile terrestrial sensing data requires TomTom to drive U.S. roads in a vehicle instrumented with highly accurate remote sensing devices, including GPS, differential GPS, inertial measurement unit, gyroscopes, and accelerometers. The data are filtered and 
synthesized using TomTom's proprietary methodology to produce road grade estimates, which are linked to a digital road network. The majority of grade data from major U.S. highways is produced using this method.

For roads where TomTom has yet to achieve coverage with advanced mobile terrestrial sensing, aggregated mobile terrestrial sensing data are employed. Road grade estimates of this type are generated by spatially aligning GPS elevation readings from a high number of TomTom consumer devices. Using multiple passes over a single stretch of road, GPS recorded elevations are averaged, filtered, and differentiated using TomTom's proprietary methodology to produce road grade estimates, which are linked to the digital road network.

The TomTom ADA product contains approximately 74 million points across the United States with a nominal advertised accuracy on road grade of $\pm 0.3 \%$. This dataset includes $100 \%$ coverage on U.S. controlled access highway routes consisting of approximately 2.6 million points over 127 thousand miles of roadway (resulting in an average distance between grade points of 258 feet). Grade points are provided as instantaneous grade measurements; road grade in between published points is interpreted using linear interpolation with respect to distance. By non-uniformly spacing grade points, TomTom is able to provide a complete road grade profile while minimizing database size and query time. Figure 3 shows TomTom's national ADA road grade coverage on controlled access routes.

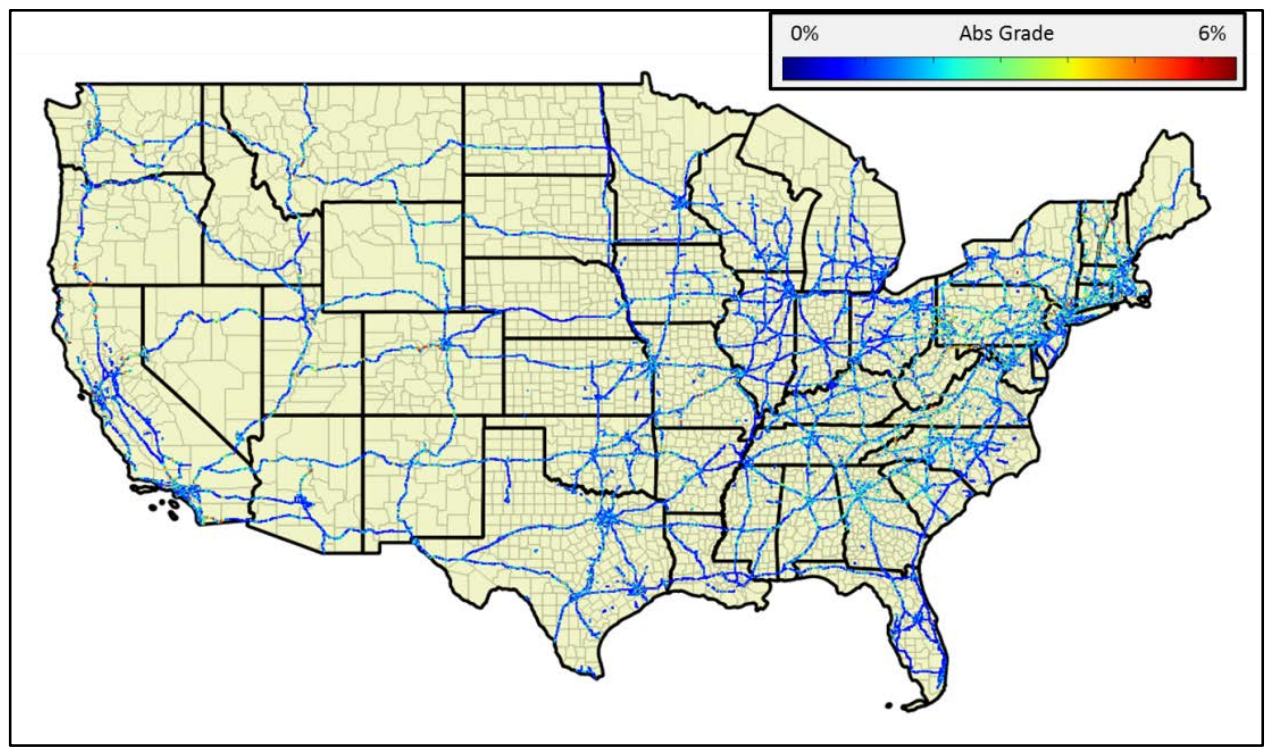

Figure 3. U.S. controlled access highways by TomTom ADA road grade

\subsection{Local Level Comparison}

Filtered USGS and TomTom data were evaluated against grade measurements from SwRI on the 115-mile east-bound stretch of I-10 in Texas from Junction to San Antonio. Figure 4 shows a full USGS elevation map of the region with the I-10 highway trace overlaid in black. 


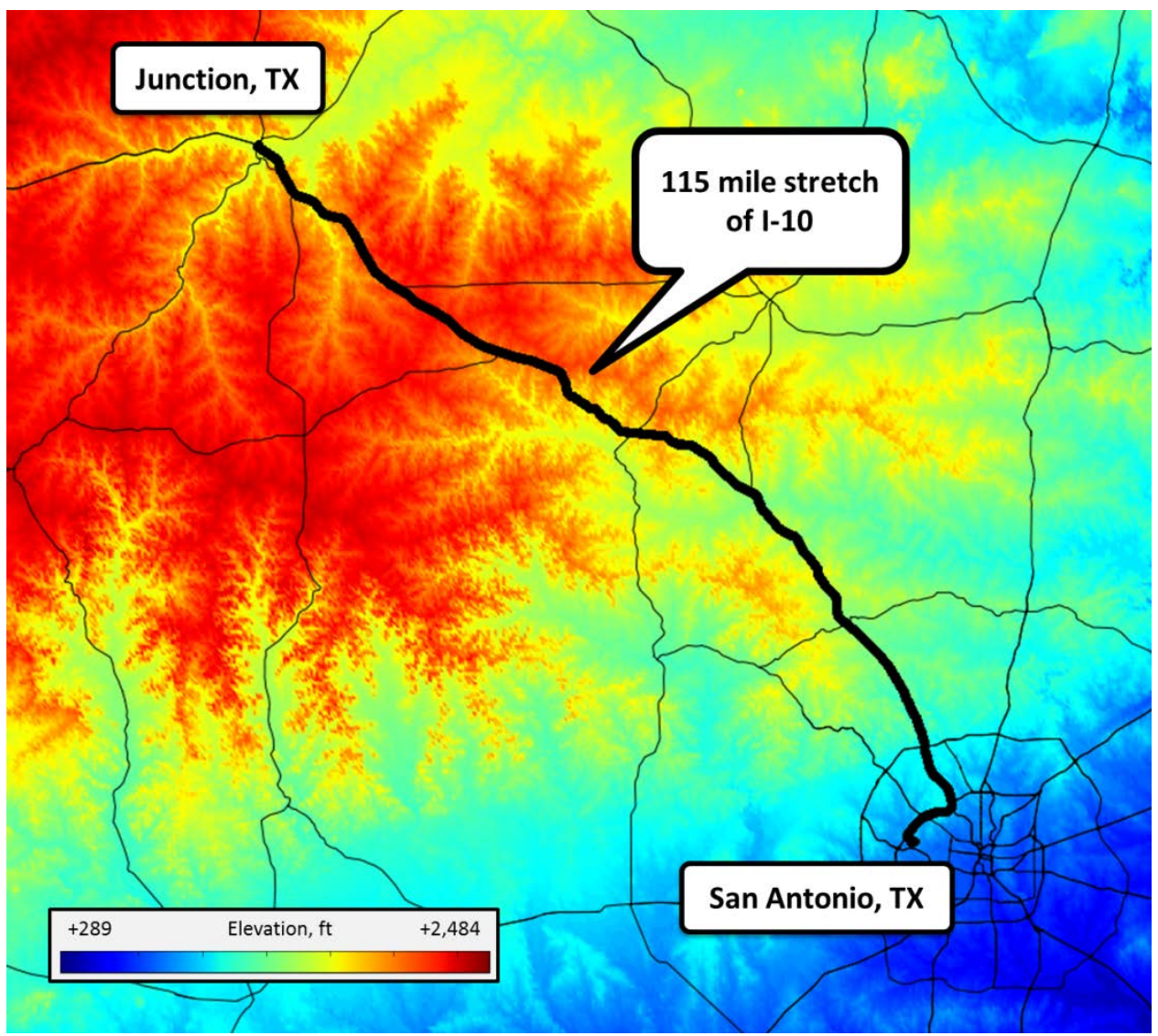

Figure 4. USGS elevation map near San Antonio, Texas, overlaid with GPS trace of MARTI vehicle driving from Junction, Texas, to San Antonio, Texas, on I-10

\subsubsection{Filtered USGS versus SwRI}

Figures 5, 6, and 7 graphically compare filtered USGS elevation and grade relative to SwRI data (plotted against distance, running west to east). Relative elevation from the SwRI data is calculated by integrating measured grade versus distance and adding an offset such that the SwRI elevation matches USGS data at mile zero. Raw USGS elevations are filtered and smoothed using NREL's standard processing routine and plotted as USGS filtered elevations. This filtering routine has a relatively small impact on elevation values and is implemented primarily to produce a clean derivative. USGS road grade is calculated by differentiating the filtered USGS elevations with respect to distance.

Figure 5 graphically compares SwRI and filtered USGS elevation and grade values. This plot shows that elevation values agree well for the majority of the 115-mile stretch in question. This provides confidence that the SwRI grade data are free of bias that would cause the integrated grade signal to drift over the 115 -mile route. 

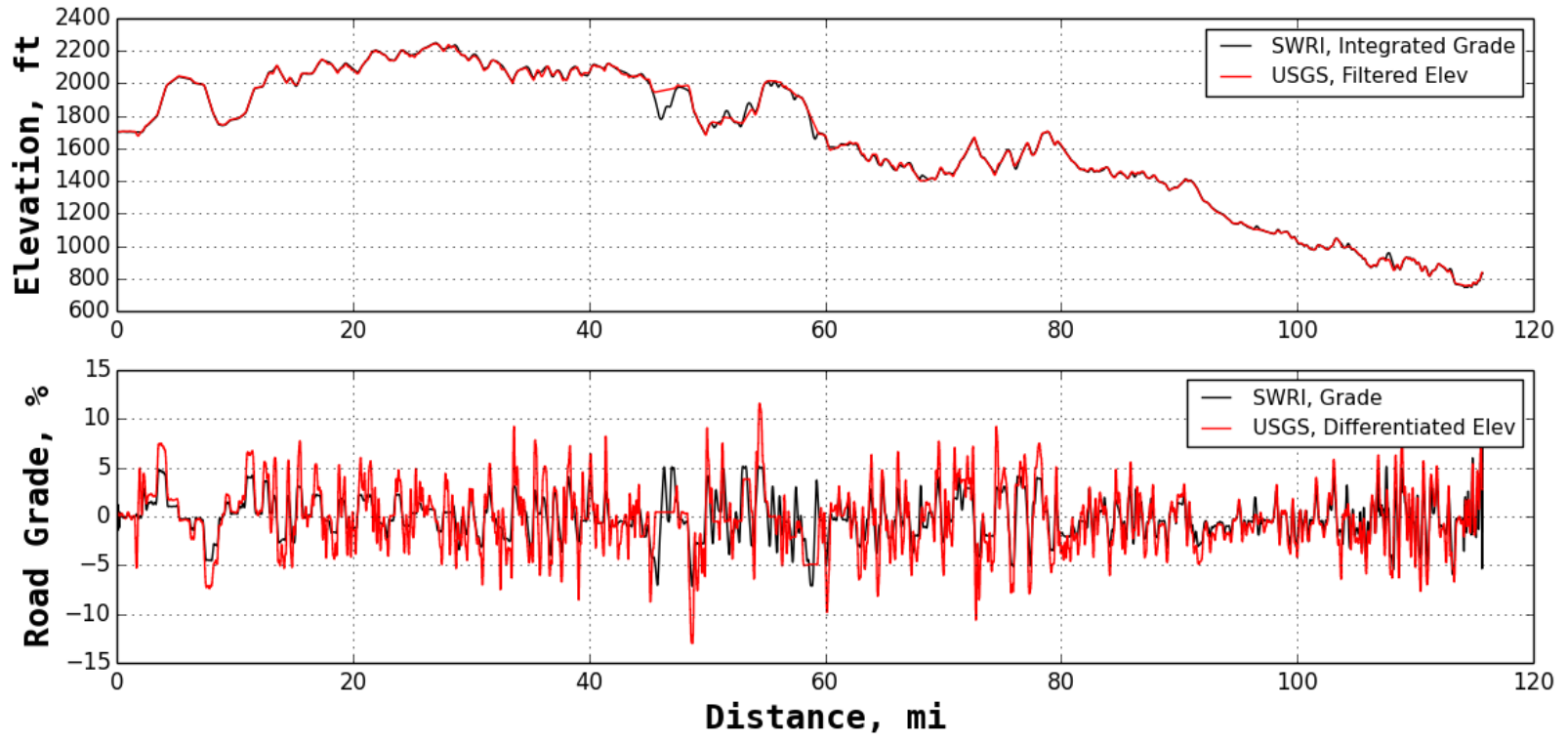

Figure 5. SwRI/USGS comparison over the 115-mile stretch of I-10 northwest of San Antonio, Texas. Top: SwRI integrated grade plotted against filtered USGS elevation. Bottom: SwRI grade plotted against filtered, differentiated USGS elevation.

Figure 6 is a zoom of the SwRI/USGS comparison from miles 97 to 104. At this magnification, we can now see that the filtered, differentiated USGS elevation agrees generally well with the SwRI grade measurements.
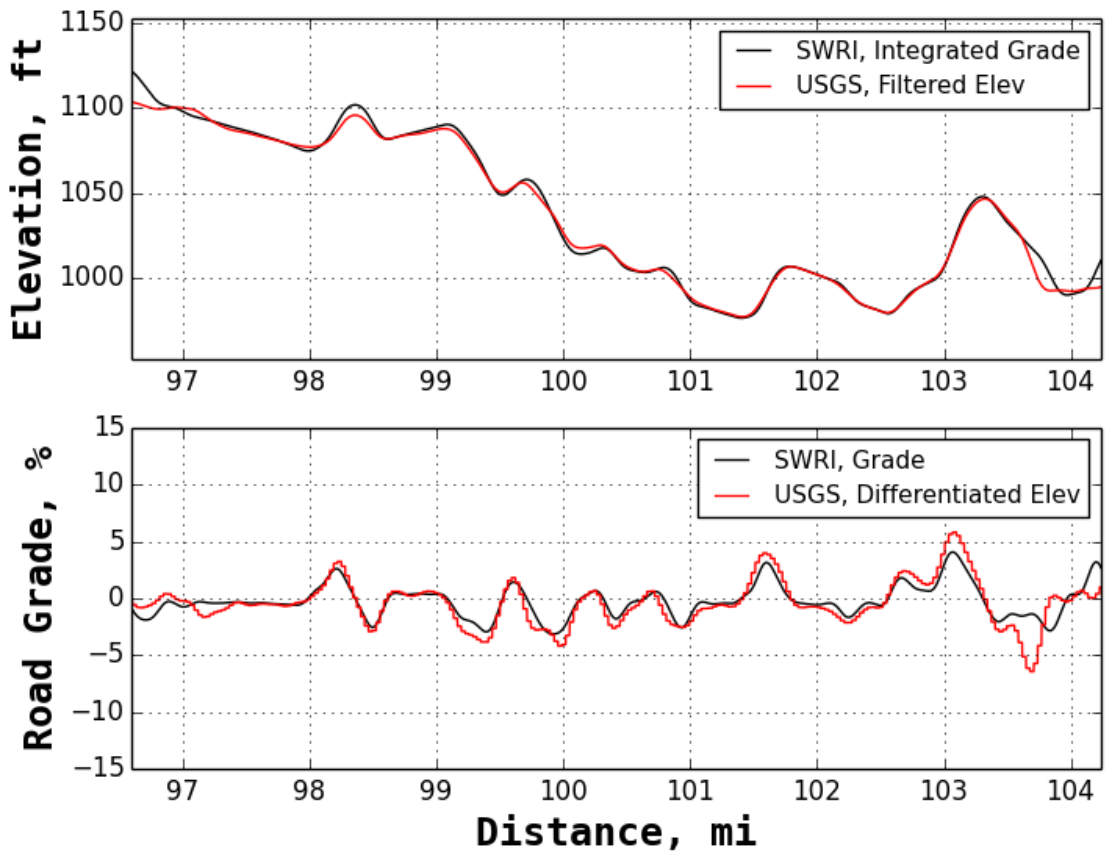

Figure 6. SwRI/USGS comparison over a subset of the 115-mile stretch of I-10 northwest of San Antonio, Texas. Top: SwRI integrated grade plotted against filtered USGS elevation. Bottom: SwRI grade plotted against filtered, differentiated USGS elevation. 
In Figure 7, all grade measurements from the SwRI and filtered USGS datasets are scattered against one another. (The density of this scatter prompted applying a level of visual transparency to each marker to get a better sense of density.) All grade values would fall exactly on the red dashed diagonal line if the datasets matched exactly. Visual inspection reveals that the filtered USGS data generally result in grades of greater magnitude relative to the SwRI data with a calculated RMSE of $1.9 \%$. This suggests that the 2014 version of NREL's processing routine is potentially under-filtering raw USGS elevations in this region of the country.

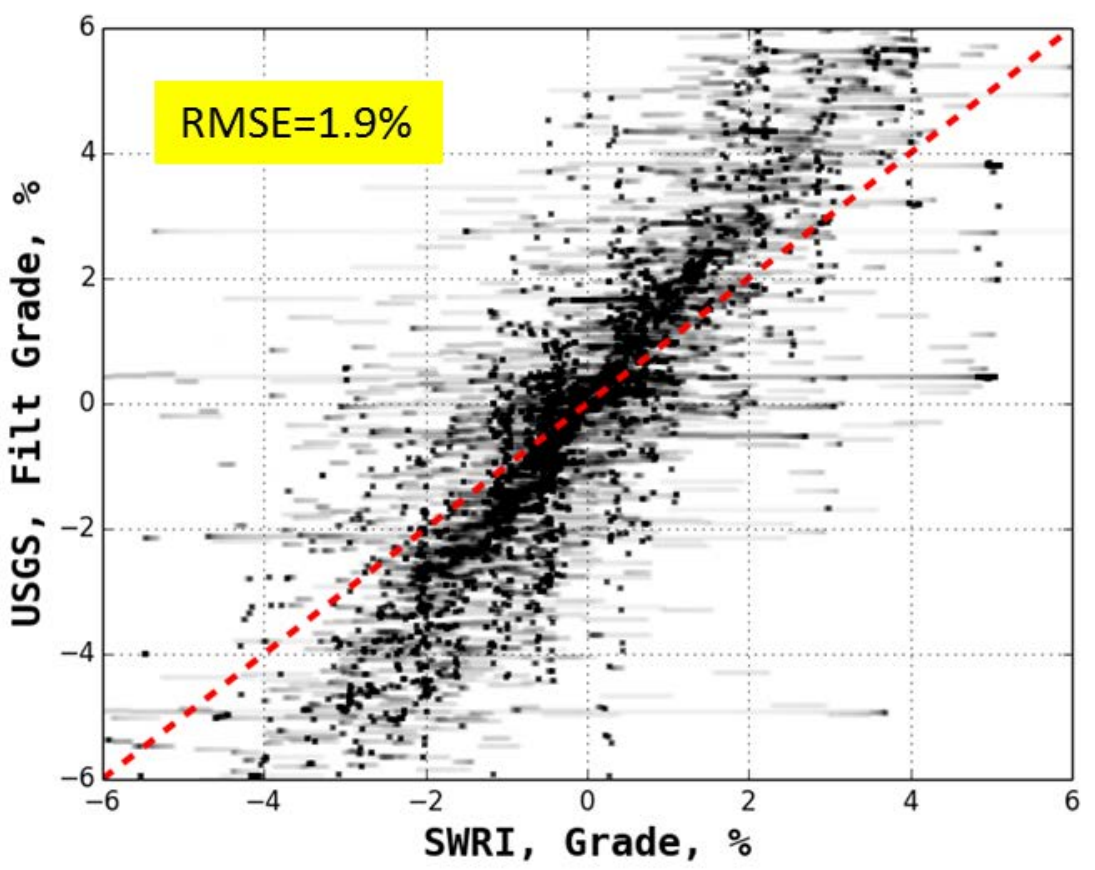

Figure 7. Filtered USGS grade scattered against SwRI grade from the 115-mile stretch of I-10 northwest of San Antonio, Texas

Dashed diagonal line provided as reference for exact agreement

\subsubsection{TomTom versus SwRI}

Figures 8, 9, and 10 graphically compare TomTom grade data relative to SwRI data (plotted against distance, running west to east). Because the TomTom database reports only road grade values, the TomTom grade data are integrated to calculate a relative elevation and offset to match SwRI's integrated grade at mile zero.

Figure 8 provides a graphical comparison of SwRI and TomTom elevation/grade values. This plot shows that TomTom integrated grade values generally agree well with SwRI integrated grade. Just before mile 80, the integrated TomTom grade exhibits a slight offset, which holds for the remainder of the 115-mile stretch. This indicates that SwRI and TomTom grade values only disagree significantly for a relatively small segment of this 115-mile stretch.

Figure 9 is a zoom of the SwRI/TomTom comparison from mile 86 to mile 93 . At this magnification, we can see that TomTom grades are nearly identical to SwRI data over this short stretch of road. Additionally, we find that TomTom grade points are reported at a frequency such 
that linear interpolation of the TomTom data also agrees well with SwRI measurements (i.e., not only do TomTom markers match SwRI data, but linear segments connecting available TomTom grades overlay well with SwRI data).
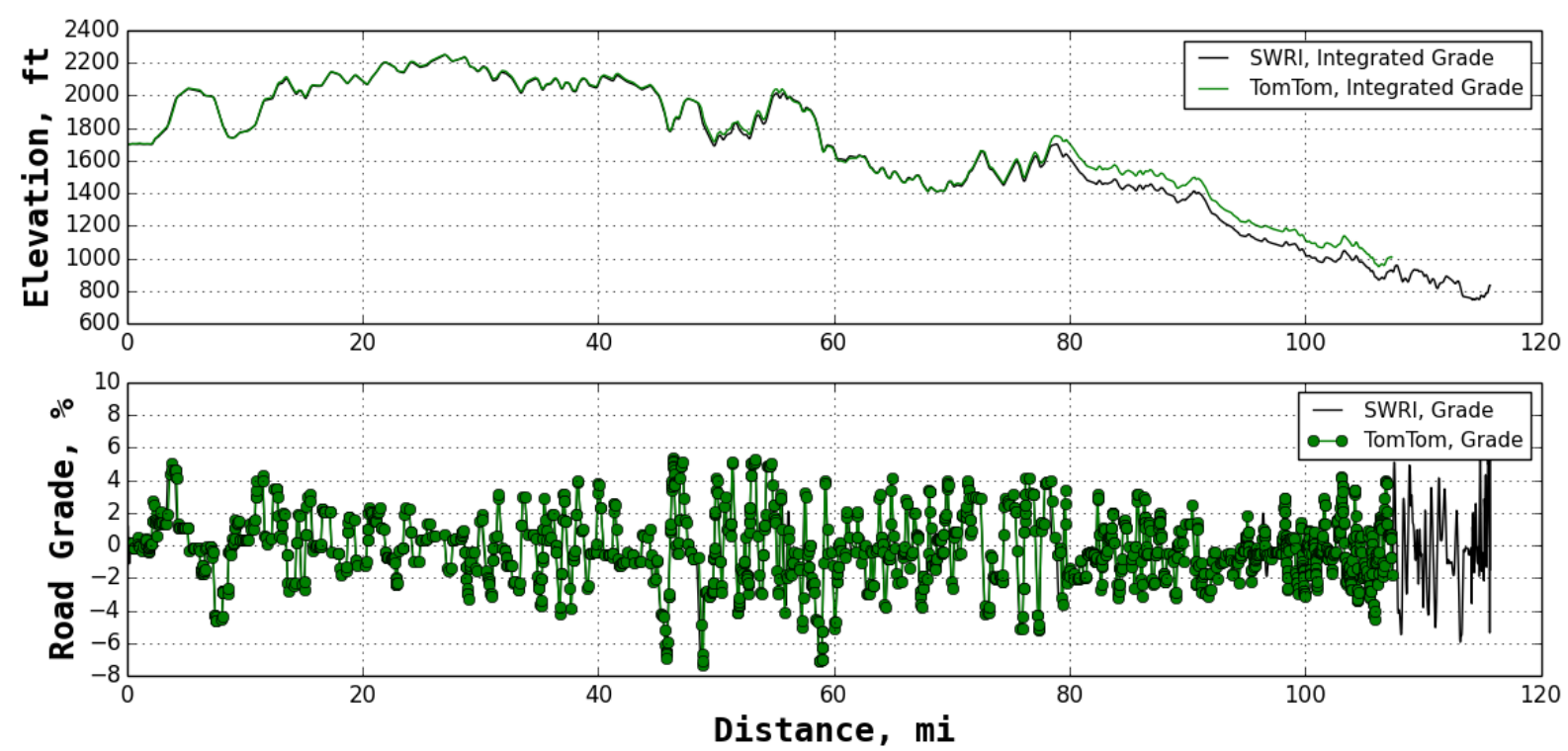

Figure 8. SwRI/TomTom comparison over the 115-mile stretch of I-10 northwest of San Antonio, Texas. Top: SwRI integrated grade plotted against integrated TomTom grade. Bottom: SwRI grade plotted against TomTom grade.
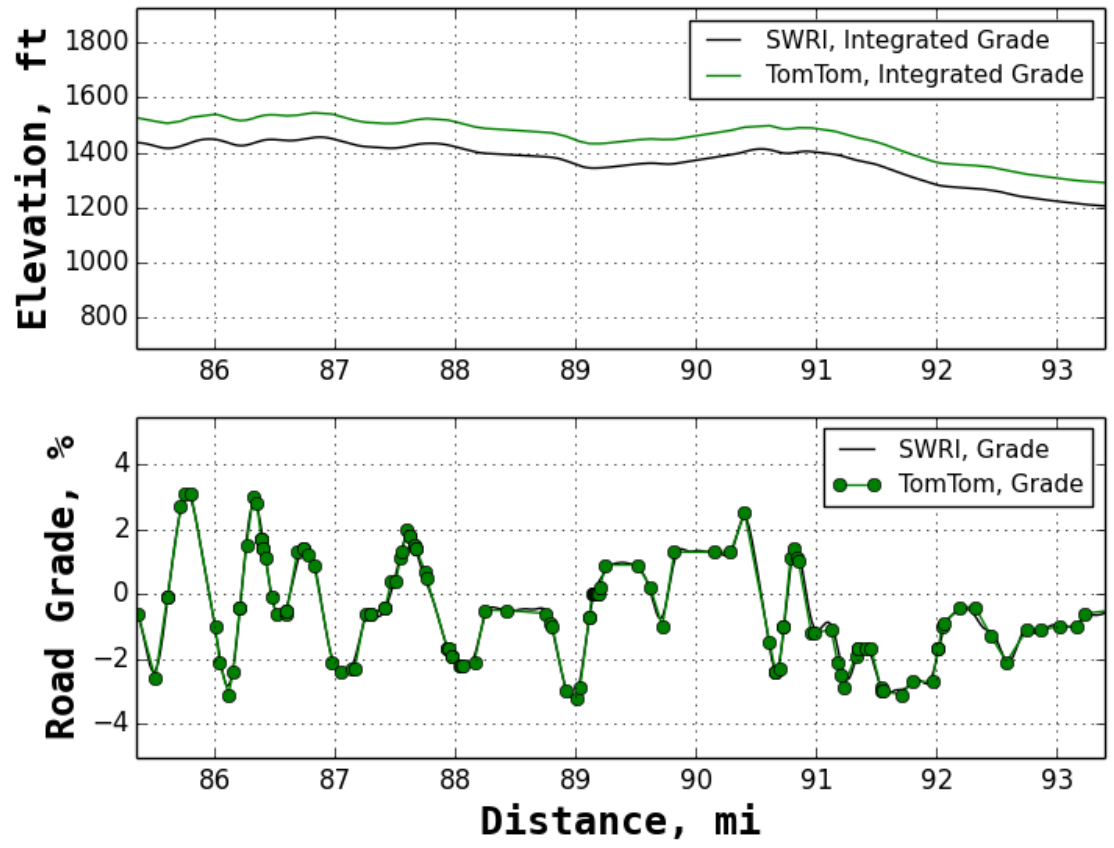

Figure 9. SwRI/TomTom comparison over a subset of the 115-mile stretch of I-10 northwest of San Antonio, Texas. Top: SwRI integrated grade plotted against integrated TomTom grade. Bottom: SwRI grade plotted against TomTom grade. 
In Figure 10, all grade measurements from the SwRI and TomTom datasets are scattered against one another. The majority of the points along the 115-mile route fall very close to the red dashed line, indicating a strong agreement between these SwRI and TomTom data. RMSE for the TomTom dataset was calculated as $0.3 \%$.

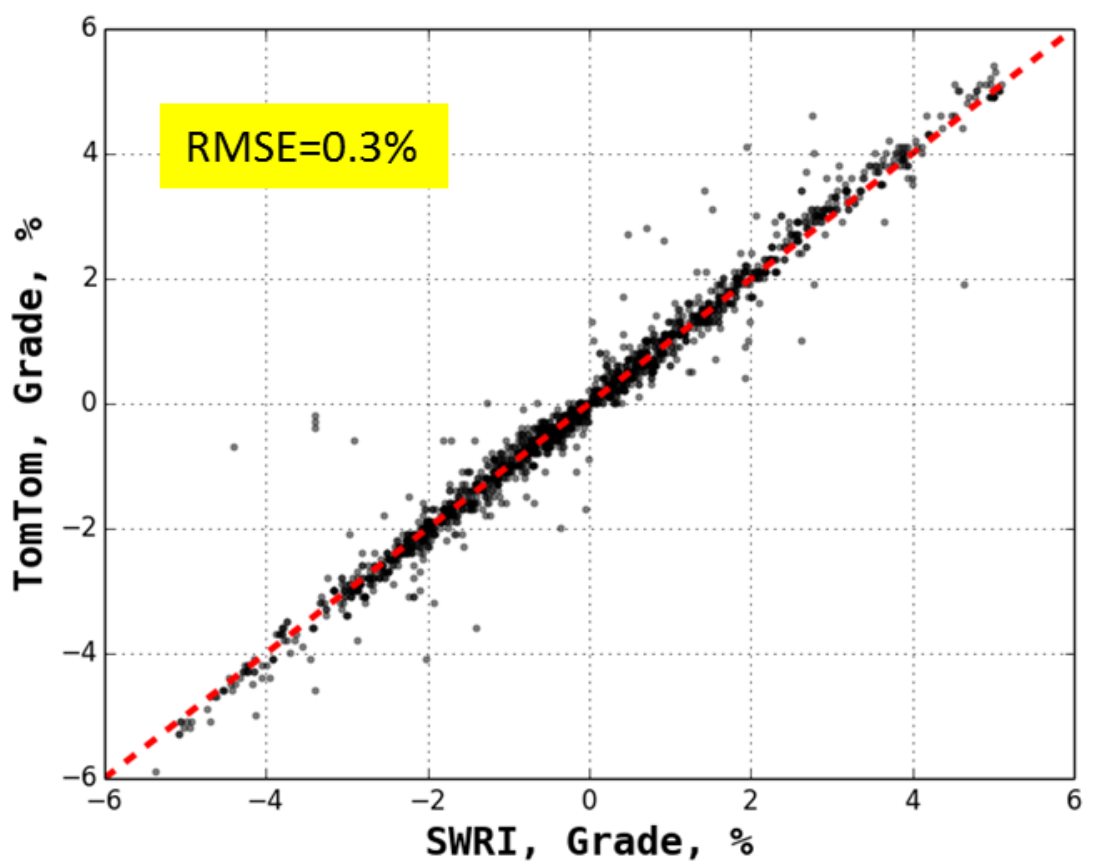

Figure 10. TomTom grade scattered against SwRI grade from the 115-mile stretch of I-10 northwest of San Antonio, Texas

Dashed diagonal line provided as reference for exact agreement

\subsubsection{Local Level Comparison Summary}

Filtered USGS and TomTom descriptions of elevation and grade along this 115-mile stretch of I-10 were compared side-by-side relative to SwRI MARTI data. Numerical evaluations of road grade accuracy are conducted at two frequencies: 1) the frequency of available data, and 2) at uniform 10-foot intervals with linear interpolation of available data. Recall that the SwRI MARTI data were collected at $100 \mathrm{~Hz}$, a higher spatial resolution than both the USGS and TomTom datasets; at a vehicle speed of $70 \mathrm{mph}$, this translates to grade readings approximately every 1 foot. Because the national databases feature larger, non-uniform measurement intervals, comparisons based on available data could potentially bias results. A visual representation of the two sampling frequencies is shown in Figure 11.

Table 1 summarizes the numerical comparison of the national datasets based on available and interpolated data. Based on available data, the SwRI measurements feature by far the highest sampling frequency per mile (nominally $100 \mathrm{~Hz}$ at highway speeds). There is an apparent tradeoff between sampling frequency and accuracy (quantified as RMSE relative to SwRI data) in the national datasets. The filtered USGS data are available at the highest spatial frequency, but also exhibit the highest RMSE; conversely, the TomTom data have the lowest RMSE, but also exhibit the lowest spatial frequency. 


\section{Example data sampled from...}

... linear interpolation of available locations at uniform intervals (0.1 miles in this illustration)

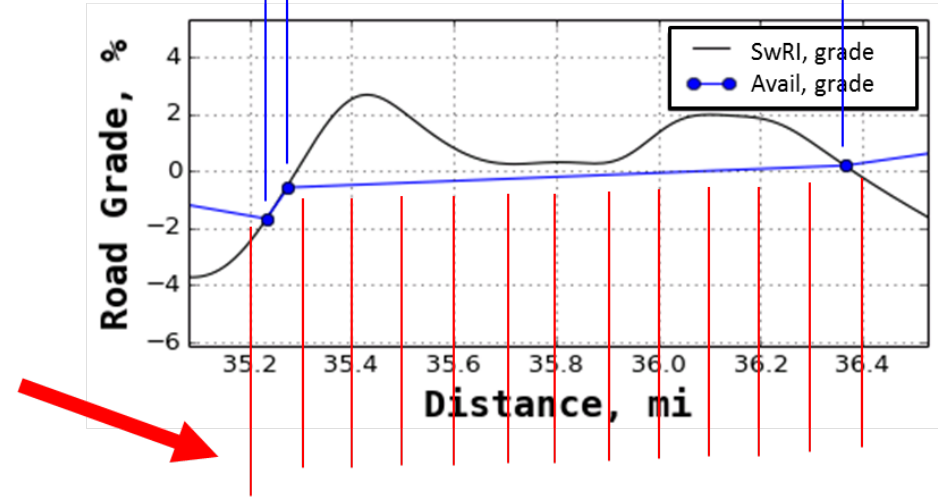

Figure 11. Visual description of the two sampling frequencies used in the comparative analysis: 1) based on available data, and 2) based on linear interpolation at uniform intervals of 10 feet (0.1 mile interval is used for illustrative purposes)

Table 1. Numerical comparison of national datasets (USGS, TomTom) relative to SwRI data from 115-mile stretch of I-10 outside San Antonio, Texas

\begin{tabular}{ll|c|cc} 
& & SwRI & USGS & TomTom \\
\hline & Distance, miles & 115.65 & 115.65 & 115.65 \\
& Points per mile & $5,636.3$ & 160.9 & 15.6 \\
Based on & Avg grade & $0.0 \%$ & $0.0 \%$ & $-0.2 \%$ \\
& Avg abs grade & $1.6 \%$ & $2.5 \%$ & $1.6 \%$ \\
& Grade RMSE & - & $1.9 \%$ & $0.3 \%$ \\
& Grade maximum absolute error & - & $8.7 \%$ & $3.7 \%$ \\
\hline \multirow{3}{*}{$\begin{array}{l}\text { Based on } \\
\text { interpolated data }\end{array}$} & Points per mile & 528 & 528 & 528 \\
& Avg grade & $-0.2 \%$ & $-0.3 \%$ & $-0.2 \%$ \\
& Avg abs grade & $1.6 \%$ & $2.5 \%$ & $1.6 \%$ \\
& Grade RMSE & - & $2.0 \%$ & $0.4 \%$ \\
& Grade maximum absolute error & - & $8.7 \%$ & $3.7 \%$ \\
\hline
\end{tabular}

Comparison evaluated at two frequencies: 1) based on available data, and 2) based on linear interpolation at uniform intervals of 10 feet.

To normalize for bias associated with sampling frequency, the datasets are resampled using linear interpolation at uniform, identical 10-foot intervals such that all datasets provide 528 grade readings per mile over the 115-mile section of I-10 outside San Antonio, Texas. Based on uniform sampling, the TomTom data clearly result in the lowest RMSE and maximum error at $0.4 \%$ and $3.7 \%$, respectively. 
These numerical findings are consistent with visual inspections that also suggested the TomTom data as the best match to SwRI measurements.

\subsection{National Level Comparison}

In addition to a rigorous local investigation on I-10 near San Antonio, Texas, the filtered USGS and TomTom datasets were evaluated at the national level. For this analysis, a national distribution of road grade was generated from the filtered USGS and TomTom databases based on available data from controlled access routes. These distributions are shown in Figure 12 as percent of highway miles at a given absolute road grade. While road grade is necessarily represented as a signed value in testing procedures and in modeling exercises, at the national level the distribution of road grade by distance using signed values is symmetrical about zero percent road grade. As such, national road grade distributions can be fully described through the use of absolute values for ease of interpretation. For additional discussion on representing road grade distributions in terms of cumulative distributions of absolute values, please refer to Appendix Section A.1.

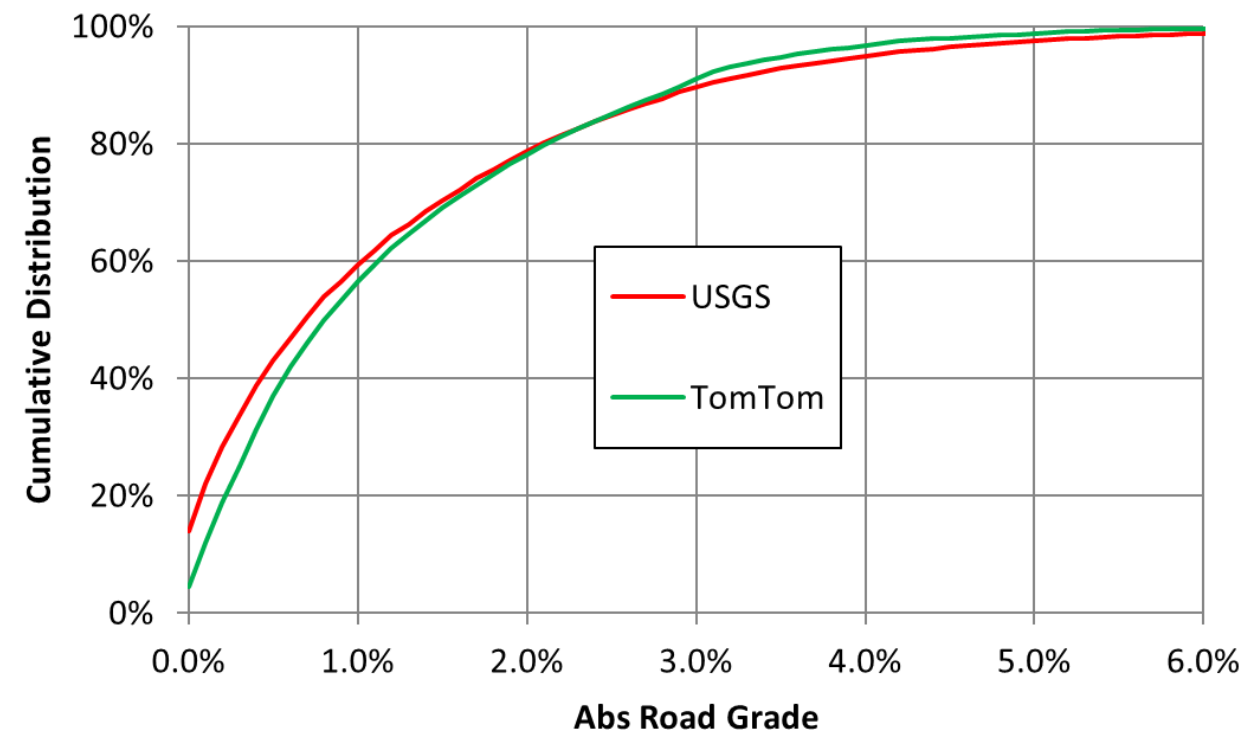

Figure 12. National distributions of road grade from filtered USGS and TomTom databases on the basis of absolute values

For the national analysis, we elected not to declare either dataset as "ground-truth" as was done with the SwRI data during inspection of the local route. Instead, the agreement between the national datasets is quantified at various levels of road grade. Visually, both national datasets follow the intuitive trend of having the majority of highway distance at relatively low grades (i.e., $57 \%-59 \%$ of distance is at less than $1 \%$ grade) and a small amount of highway distance at relatively high grades (i.e., $1 \%-2 \%$ of distance is at greater than $5 \%$ grade).

Based on the superior accuracy of the TomTom data (relative to filtered USGS data) when compared with SwRI measurements over the 115-mile stretch of I-10 near San Antonio, Texas, and the similarity of roadway distance at grade between the national databases, the TomTom database was selected as the preferred road grade database for the remainder of this analysis. 


\section{National Statistics}

Using the TomTom national road grade database as the source data for this analysis, a suite of statistical distributions was developed to describe grades experienced by MD/HD trucks on controlled access highways across the United States. This suite of descriptive statistics is developed using two analytical procedures: 1) quantifying the percent of national roadway distance at grade, and 2) evaluating national highway topography by segmenting roadways into a series of "half hills" (described in section 3.2) and recording statistics such as distance, average grade, and maximum grade for each segment. Finally, activity weighting is applied to account for the national distribution of $\mathrm{MD} / \mathrm{HD}$ vehicle highway traffic.

As this analysis is intended to support the highway testing procedure, the U.S. highways to be examined are presented first. Figure 13 shows a map of the United States overlaid with controlled access roads per the TomTom MultiNet street network. This road network includes the U.S. interstate system (shown in red) in addition to a number of locally controlled access highways (shown in blue).

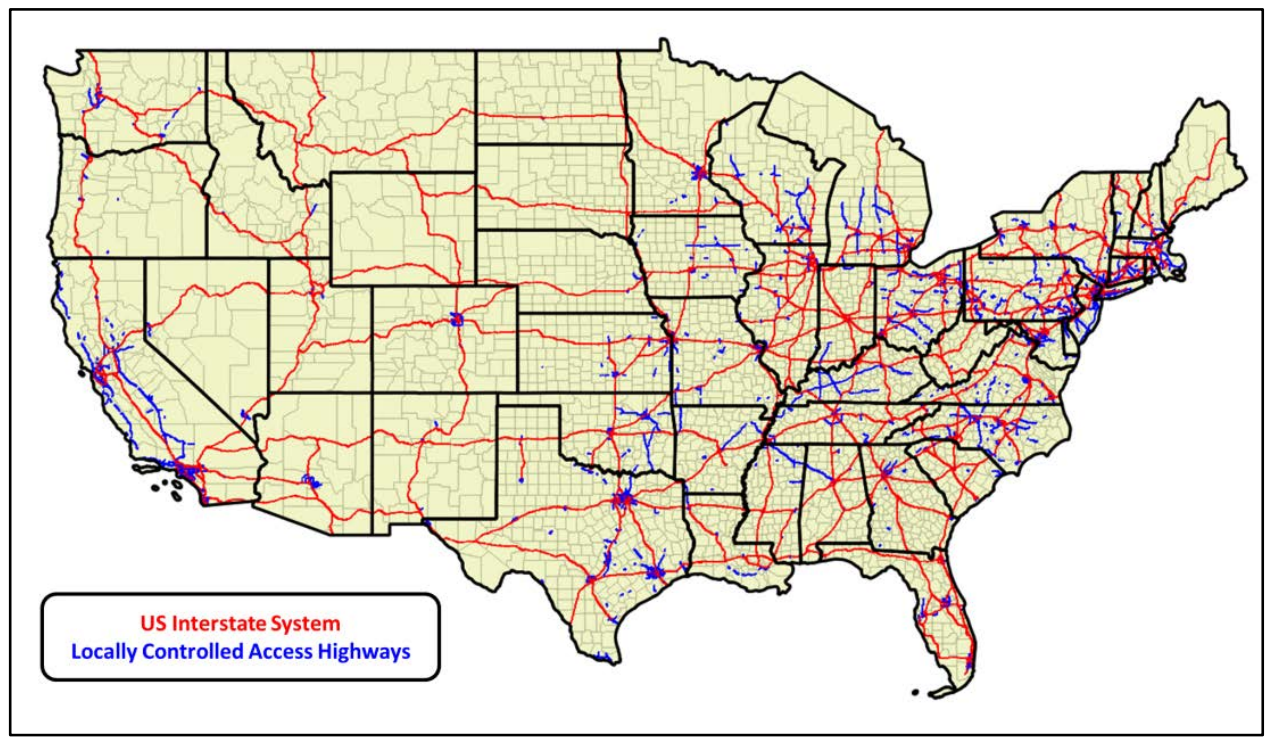

Figure 13. TomTom MultiNet map of U.S. controlled access highways Interstate highway system shown in red; locally controlled access highways shown in blue. 
Additionally, we would like to understand if road grade distributions feature significant variation when segmented by truck speed limits so as to align data generated in this report with EPA's existing 55- and 65-mph constant-speed drive cycles. Figure 14 shows the same U.S. controlled access highway map colored by state-defined truck speed limits per TomTom's Logistics data layer [26]. While truck classifications can vary by state, vehicles subject to truck speed limits generally fall in weights classes $7-8$, feature gross vehicle weight ratings of over 26,000 pounds, and have three or more axles.

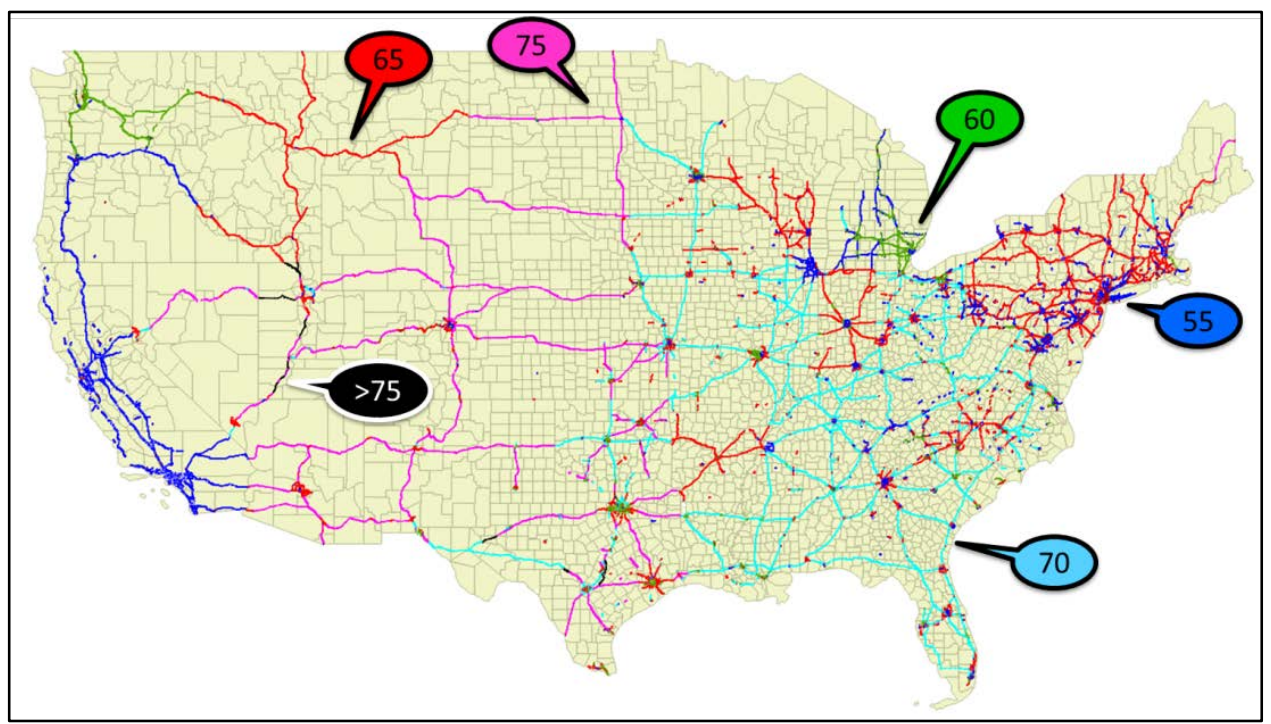

Figure 14. U.S. controlled access highway map colored by state-defined truck speed limits per TomTom Trucking Logistics speed restrictions data layer

Colored bubbles indicate truck speed limits in units of miles per hour. 
Figure 15 offers a breakdown of U.S. controlled access highway miles by truck speed limit and by U.S. Census Urban Area boundaries. From this plot, we see that the majority of U.S. controlled access highway miles come from routes with truck speed limits from 55- to 75-mph. Roadways with truck speed limits less than 55-mph are discarded on the basis that vehicle operation on such roads would likely be occurring in congested urban areas and would not be constant speed. Roadways with truck speed limits greater than 75-mph are discarded on the basis that these only occur in a very small area of the country, do not appear to be representative of national roadways, and their omission would not have a meaningful effect on the results. Therefore, the remainder of this analysis will focus on roadways in these five truck speed limit bins.

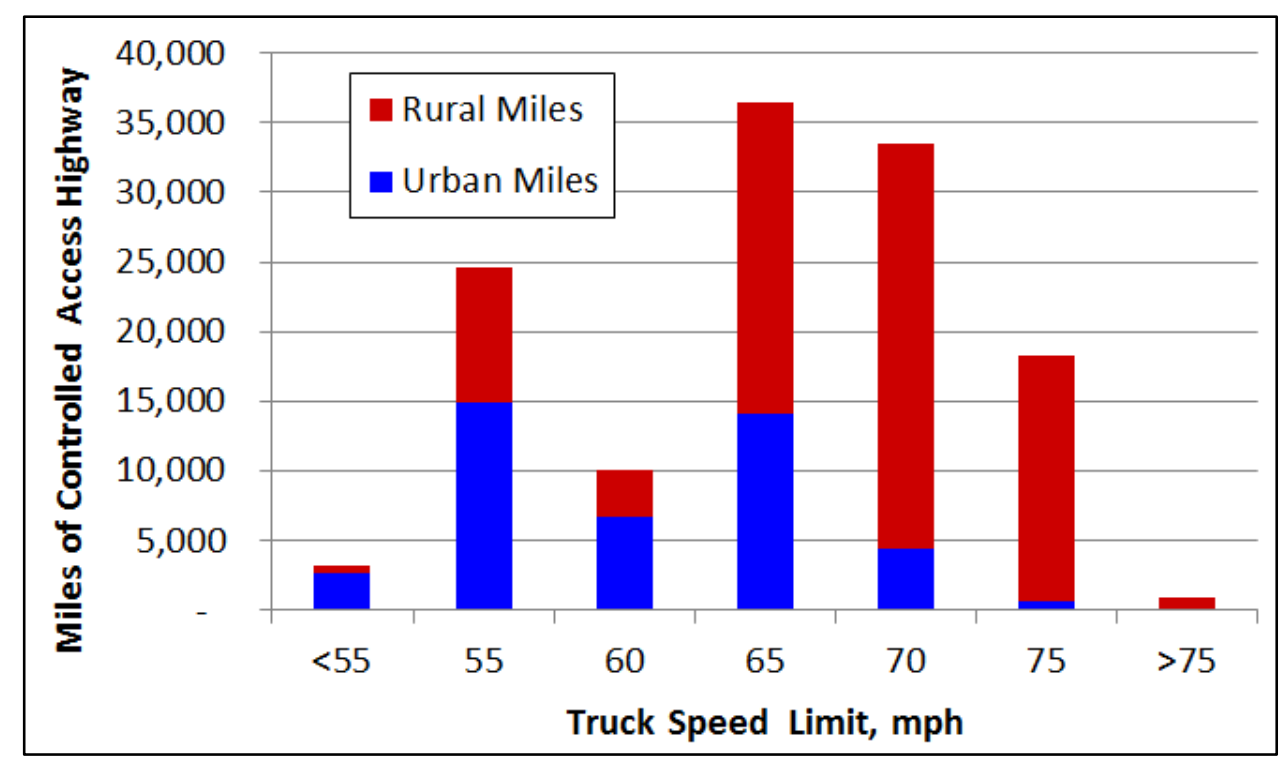

Figure 15. Mileage breakdown of U.S. controlled access highways by truck speed limit and U.S. Census urban area designation

\subsection{Road Grade by Distance Analysis}

The TomTom ADA road grade database was queried for all U.S. controlled access roads at uniform 52.8-foot intervals (100 points per mile) using linear interpolation. This procedure quantifies the percent of national highway distance at grade (distance in this analysis is defined as the horizontal component of roadway distance as described in Appendix Section A.2). Given that there are approximately 127 thousand miles of U.S. controlled access highway, these calculations result in generation of approximately 12-million road grade measurements. The data are aggregated by five truck speed limits and 1,728 U.S. counties with controlled access highways to produce 8,640 distributions of grade by distance. 
Figure 16 plots the data as cumulative distributions for all 1,728 U.S. counties in question (grade by truck speed limit is summed by county for this plot). While the density of data on this plot provided little in the form of analytical value, it is informative to understand the dramatic variability in road grade experienced by trucks in different regions of the country.

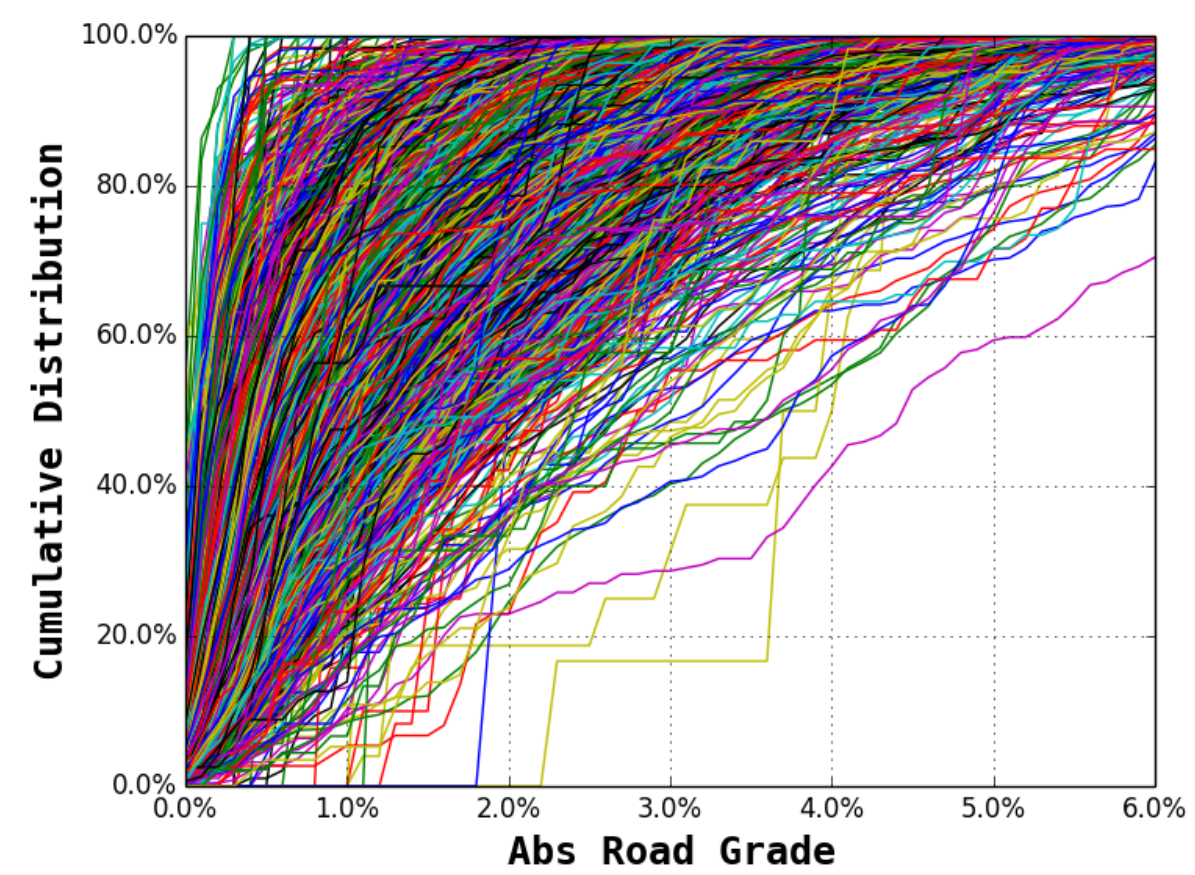

Figure 16. Cumulative distributions of road grade by distance for 1,728 U.S. counties 
In Figure 17, the 8,640 distributions are summed by truck speed limit with all data points having equal value within each truck speed limit segment (effectively resulting in distance weighting). A trend emerges from this plot that roadways with high truck speed limits generally have lower concentrations of road grade relative to roadways with low truck speed limits. For example, approximately $78 \%$ of distances on highways with truck speed limits of 55-mph exhibit less than $2 \%$ absolute road grade. That figure jumps to approximately $87 \%$ for highways with truck speed limits of 75-mph.

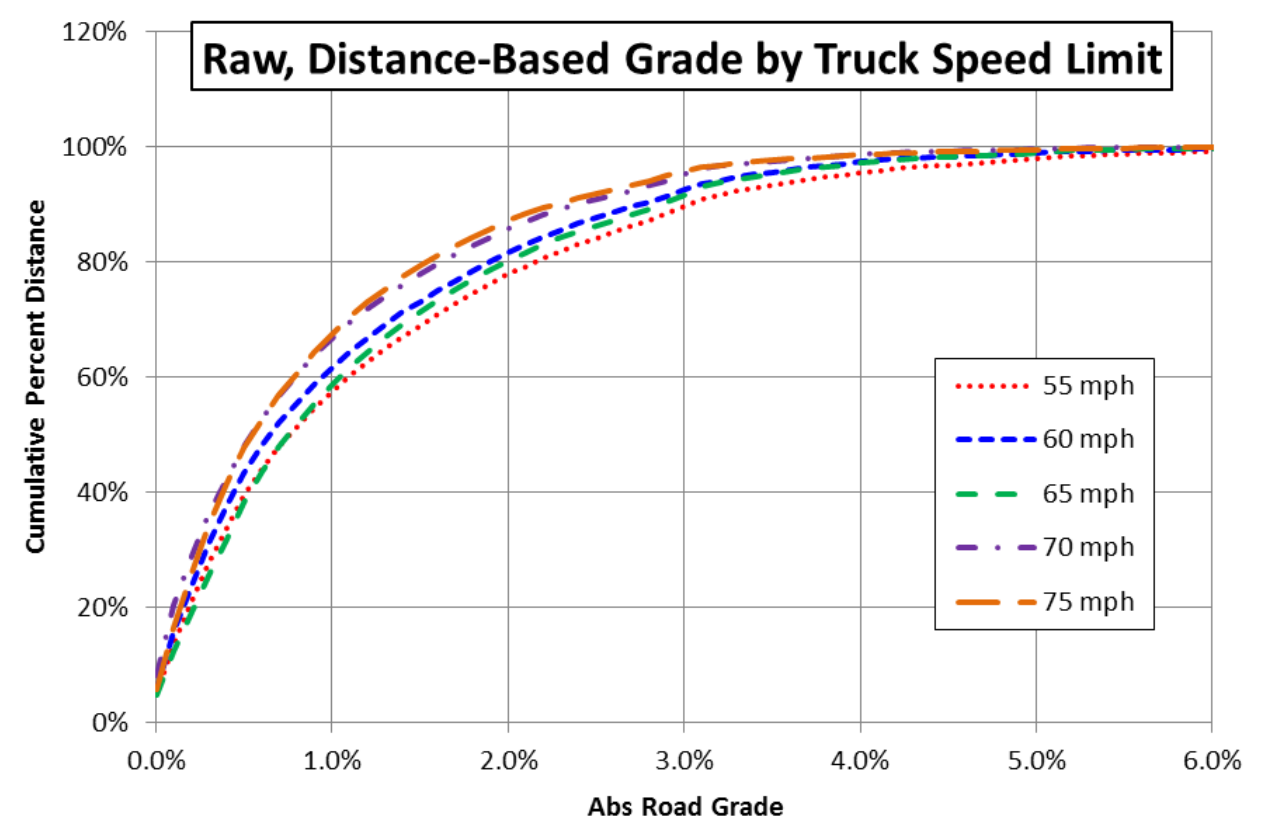

Figure 17. Raw distance-based cumulative distribution of absolute road grade by truck speed limit

\subsection{Half Hill Analysis}

While querying the national road grade database by distance is informative, additional information can be gleaned. Our second round of analysis consisted of evaluating national highway topography by segmenting roadways into a series of "half hills" and recording statistics for each segment.

A half hill in this analysis was defined as a continuous stretch of roadway where grade does not change sign (i.e., sections of exclusive climb or descent). Figure 18 shows this half hill segmenting where a 13-mile stretch of controlled access highway is subdivided into 16 unique half hills (eight climbs and eight descents). A number of statistics are recorded for each identified half hill, including distance, average grade, and maximum grade (minimum grade for segments of descent). This processing routine is applied to the entire U.S. controlled access highway system and results in generation of statistics for approximately 220 thousand half hills nationwide. 


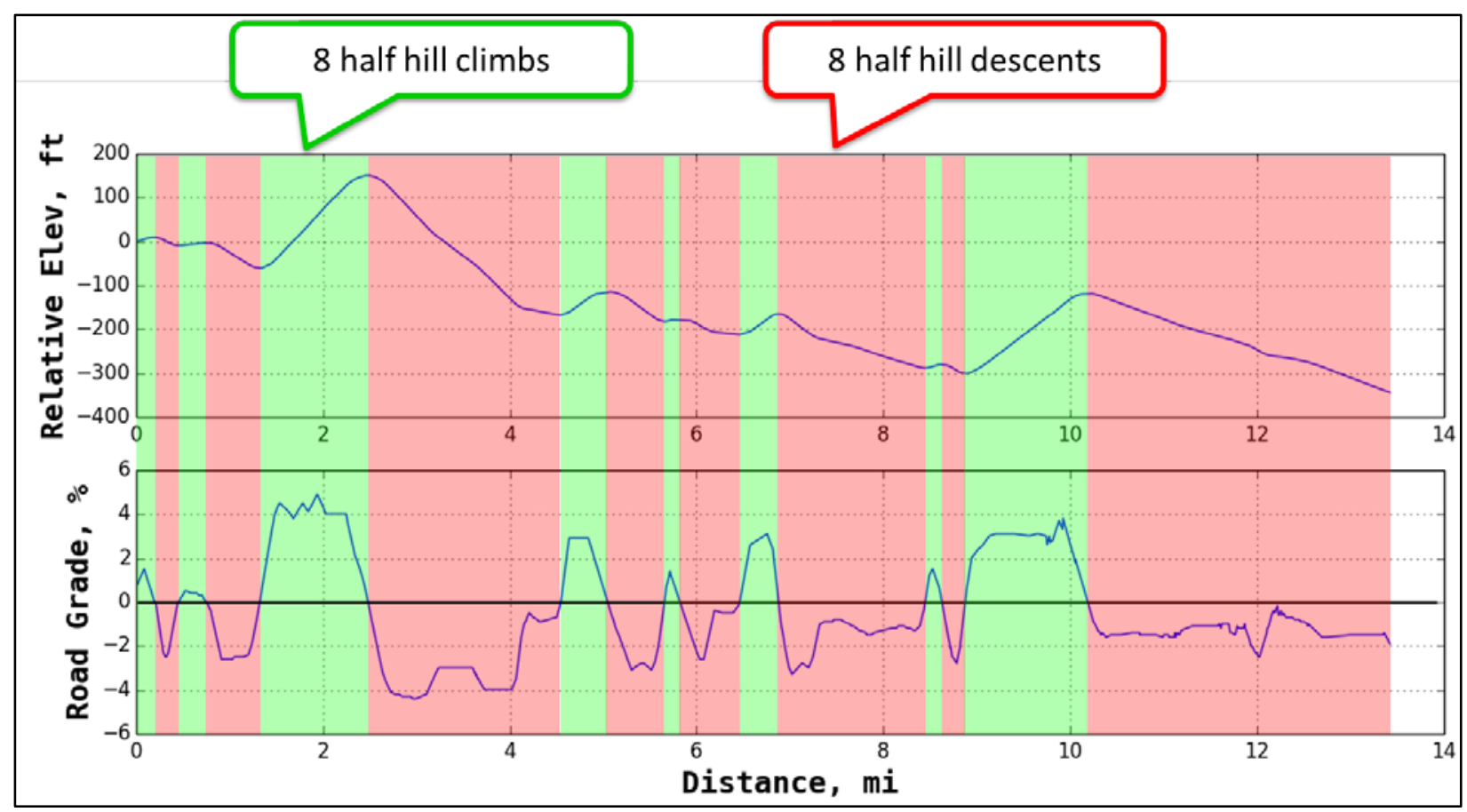

Figure 18. Example section of controlled access highway broken into 16 half hills (eight climbs and eight descents)

\subsubsection{Analytical Approaches}

During internal reviews of half hill data, we discovered the potential for misinterpretation of results when not explicitly stating the method by which our distributions were compiled. Two methods for aggregating half hill data were used: 1) frequency-based distributions, and 2) distance-based distributions. In the previous analysis of grade by distance in Section 3.1, frequency-based and distance-based interpretations carried the same meaning because the distance between data points was assigned a uniform distance of 52.8 feet. Due to the nonuniform nature of half hill distances, frequency-based and distance-based distributions of half hill data result in dramatically different statistics. For example, Figure 19 presents frequencybased and distance-based distributions of distances from the entire half hill dataset. 


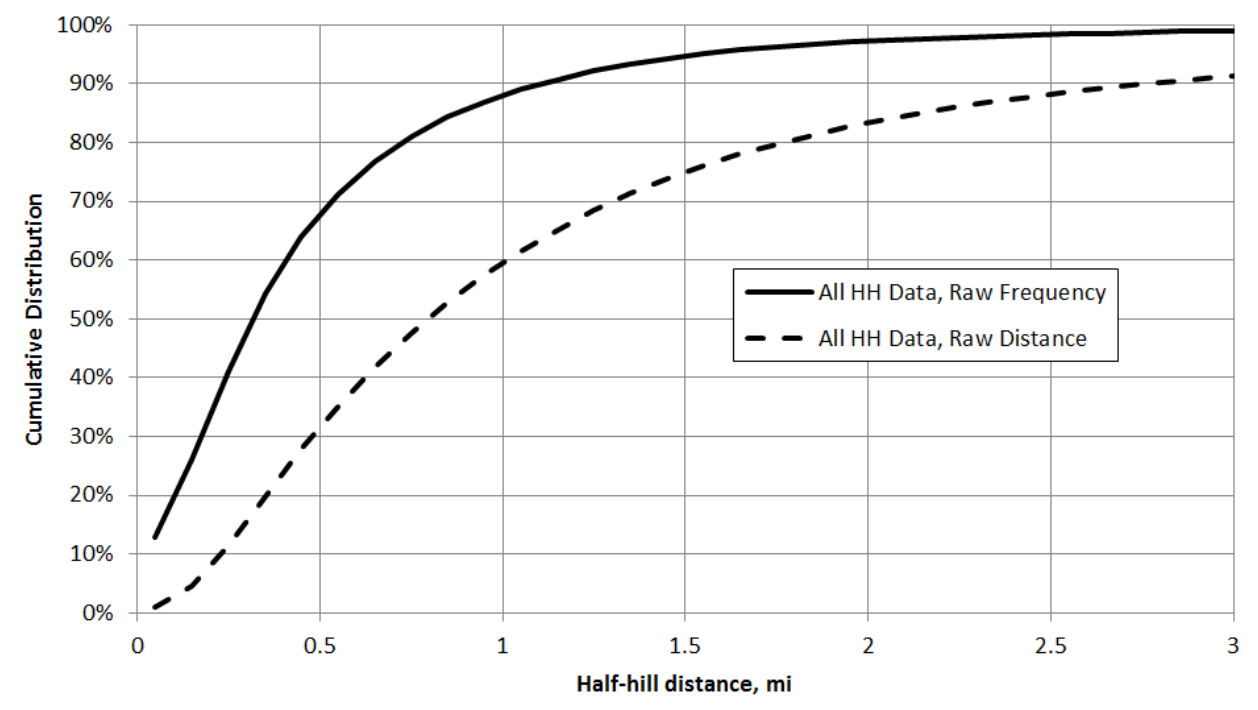

Figure 19. All half hill $(\mathrm{HH})$ distances plotted as frequency-based and distance-based cumulative distributions

The frequency-based and distance-based cumulative distributions, $\mathrm{P}$, of half hill distance, $\mathrm{d}$, are generated by evaluating Equations 1 and 2, respectively, over the entire range of half hill distances.

$$
\begin{aligned}
P_{\text {freq }}(d) & =\frac{1}{n} \sum_{i=1}^{n}\left\{\begin{array}{cc}
d_{i}<d & 1 \\
\text { else } & 0
\end{array}\right. \\
P_{\text {dist }}(d) & =\left(\sum_{i=1}^{n}\left\{\begin{array}{cc}
d_{i}<d & d_{i} \\
\text { else } & 0
\end{array}\right) / \sum_{i=1}^{n} d_{i}\right.
\end{aligned}
$$

In this set of equations, $d_{i}$ represents the distance of half hill, $i$, and $n$ represents the total half hill count. Using the same source data, we are able to create two types of distributions, each of which provides a unique perspective. For example, examination of the frequency-based distribution in Figure 19 shows that approximately $40 \%$ of half hills inspected have a distance of 0.25 mile or less. Conversely, the distance-based distribution shows that only $10 \%$ of inspected highway distance falls on half hills of distance 0.25 mile or less. By assigning all half hills equal weight, the frequency distribution overstates the amount of distance driven on short half hills and understates the amount of distance driven on long half hills.

While both methods for aggregating the half hill source data are believed to be valid, we adopted the distance-based distribution as the default method for the majority of this analysis. 


\subsubsection{Half Hill Trends with Respect to Speed Limits}

Figure 20 plots raw distance-based cumulative distributions of half hill distance by truck speed limit. Similar trends in half hill distances are apparent for roads with truck speed limits between 55- and 70-mph. However, half hill distances are noticeably longer on roads with truck speed limits of $75-\mathrm{mph}$. For example, approximately $70 \%$ of roadway distances on routes with $75-\mathrm{mph}$ truck speed limits occur on half hills of 2 miles or less. That figure jumps to a range of $82 \%$ $87 \%$ for routes with truck speed limits of 55 - to $70-\mathrm{mph}$.

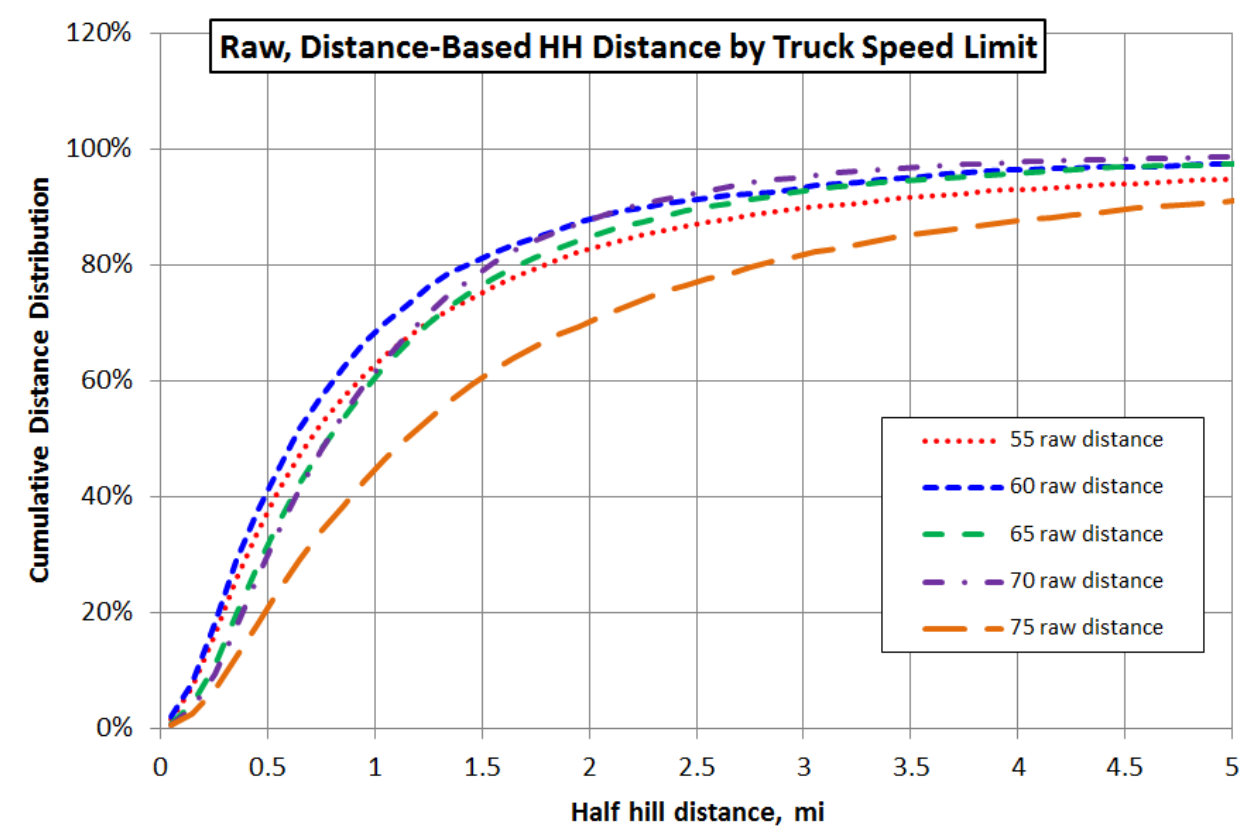

Figure 20. Raw distance-based cumulative distributions of half hill distance by truck speed limit 
Figure 21 shows national half hill data plotted by scattering maximum grade (minimum grade for segments of descent) against average grade for each half hill. The red dashed diagonal line indicates half hills where maximum and average grade are nearly equal. Overall, we find an average grade ratio (maximum over average half hill grade) of 1.76.

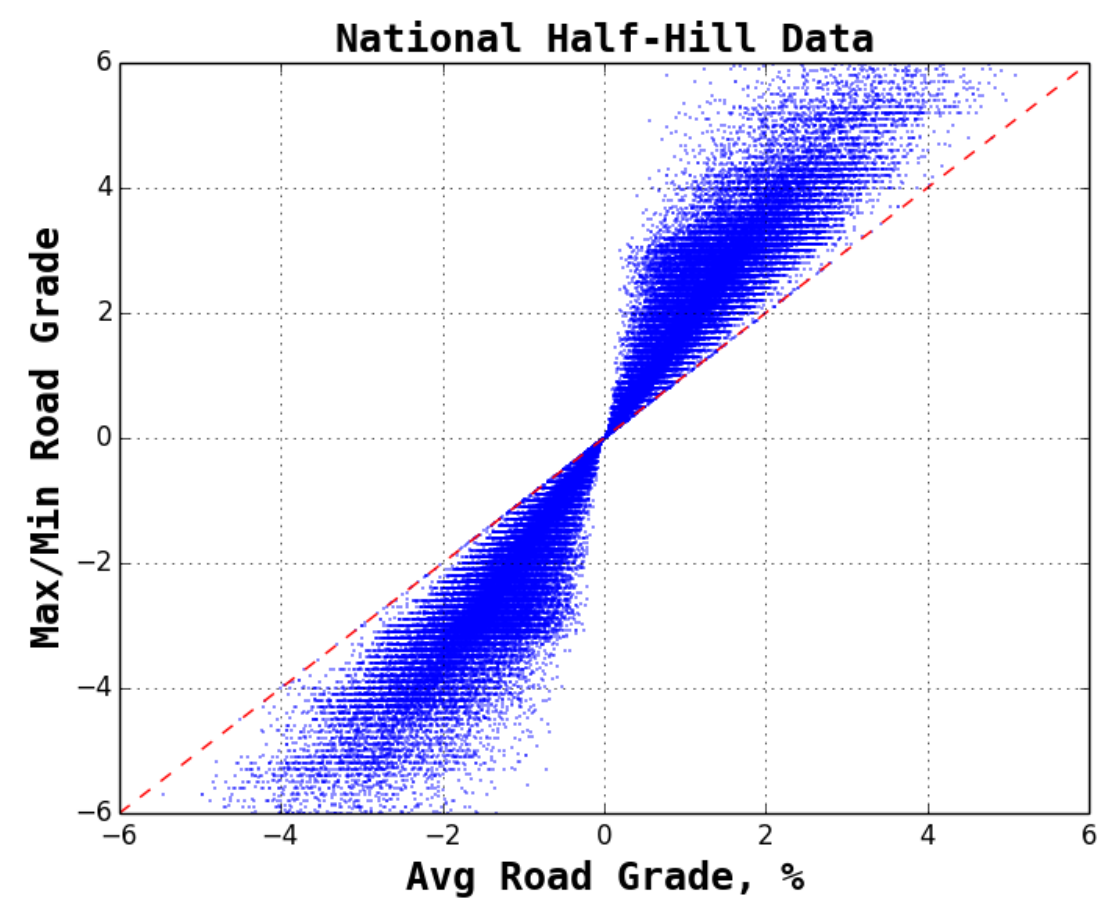

Figure 21. Maximum (or minimum) versus average road grade for all measured half hills Red dashed diagonal line shows where maximum and average grade are nearly equal. 


\subsection{Activity-Weighted Values}

Activity data for MD/HD trucks are sourced from EPA's Motor Vehicle Emission Simulator (MOVES) database of vehicle miles traveled (VMT) by county. The MOVES VMT database is summarized in Figure 22. EPA's estimates for national, annual VMT by vehicle class and road type are also included. Light-duty vehicles, including motorcycles, passenger cars, passenger trucks, and light commercial trucks, comprise the majority of activity in this database.

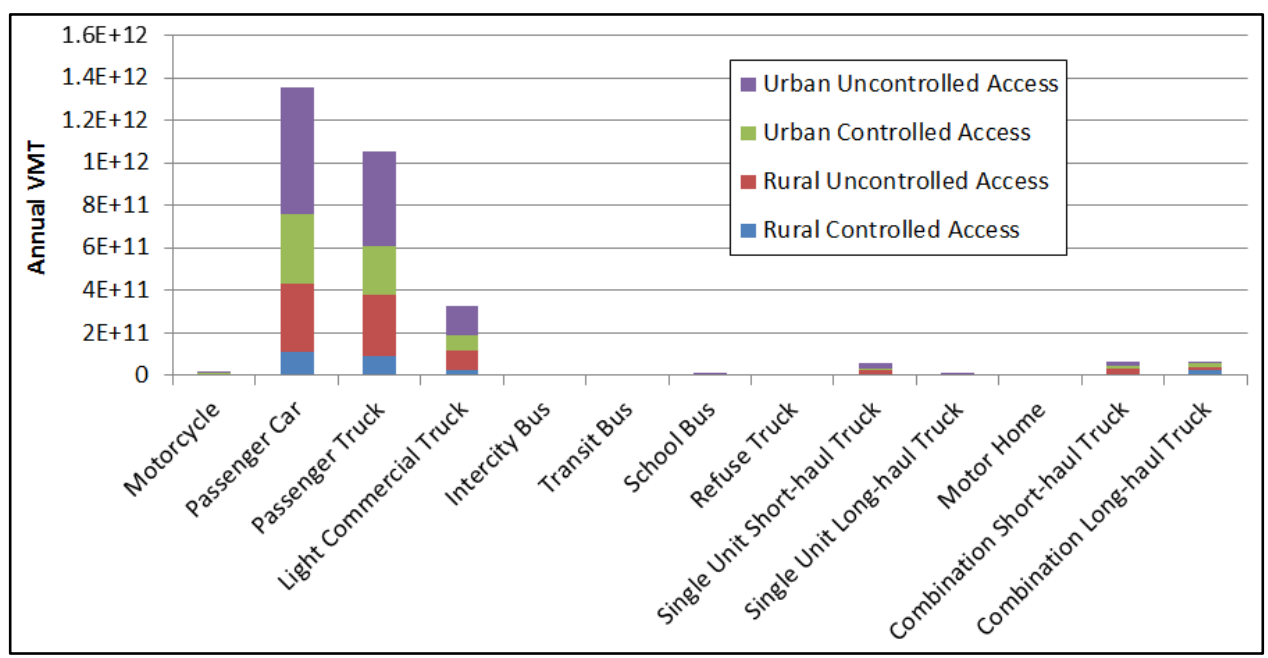

Figure 22. EPA MOVES VMT database plotted by vehicle type and roadway classification

After removing light-duty vehicle activity and VMT from uncontrolled access roads, the remaining activity data from MD/HD vehicles on controlled access roads are plotted in Figure 23. In this subset of the MOVES VMT database, the majority of activity is generated from shortand long-haul combination trucks. These are vehicles likely to fall into weight classes 7-8 and thus are subject to the truck speed limits discussed above.

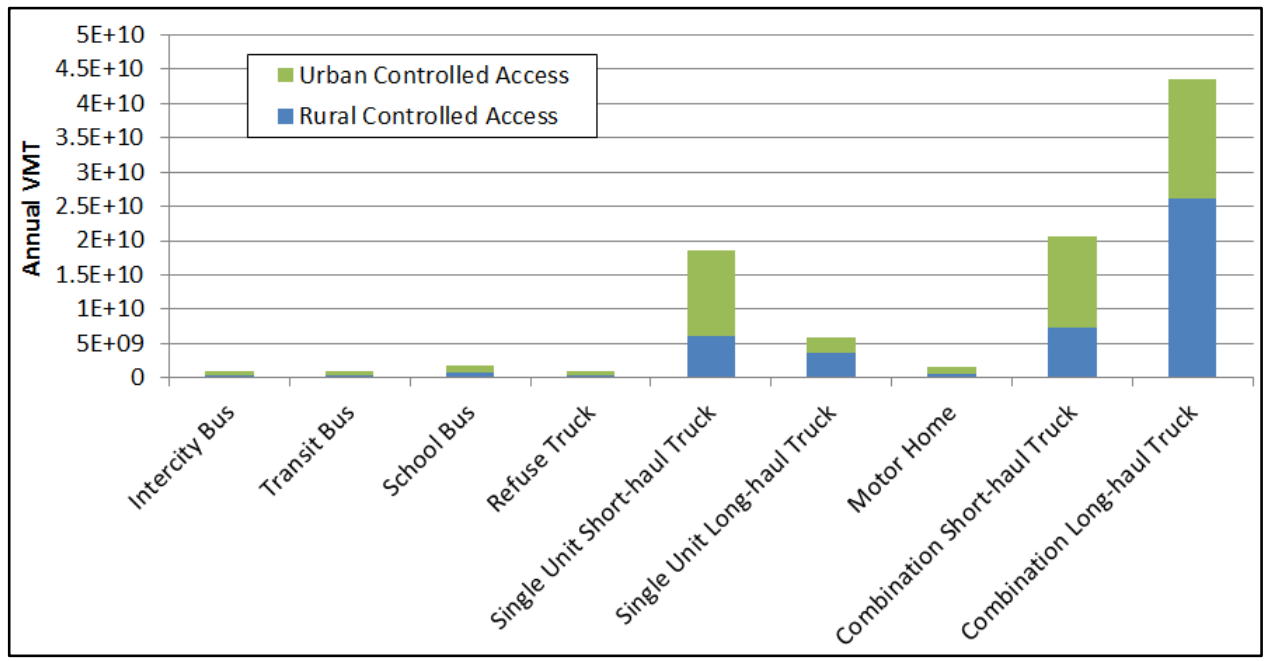

Figure 23. EPA MOVES VMT database for MD/HD vehicles on controlled access roads only 
MOVES VMT data for the subset of MD/HD vehicles on controlled access roads are spatially plotted by county in Figure 24 to better understand regional variability with respect to MD/HD truck traffic. This map reveals concentrations of activity in urban centers and on select interstate highway systems primarily oriented in the eastern third of the United States.

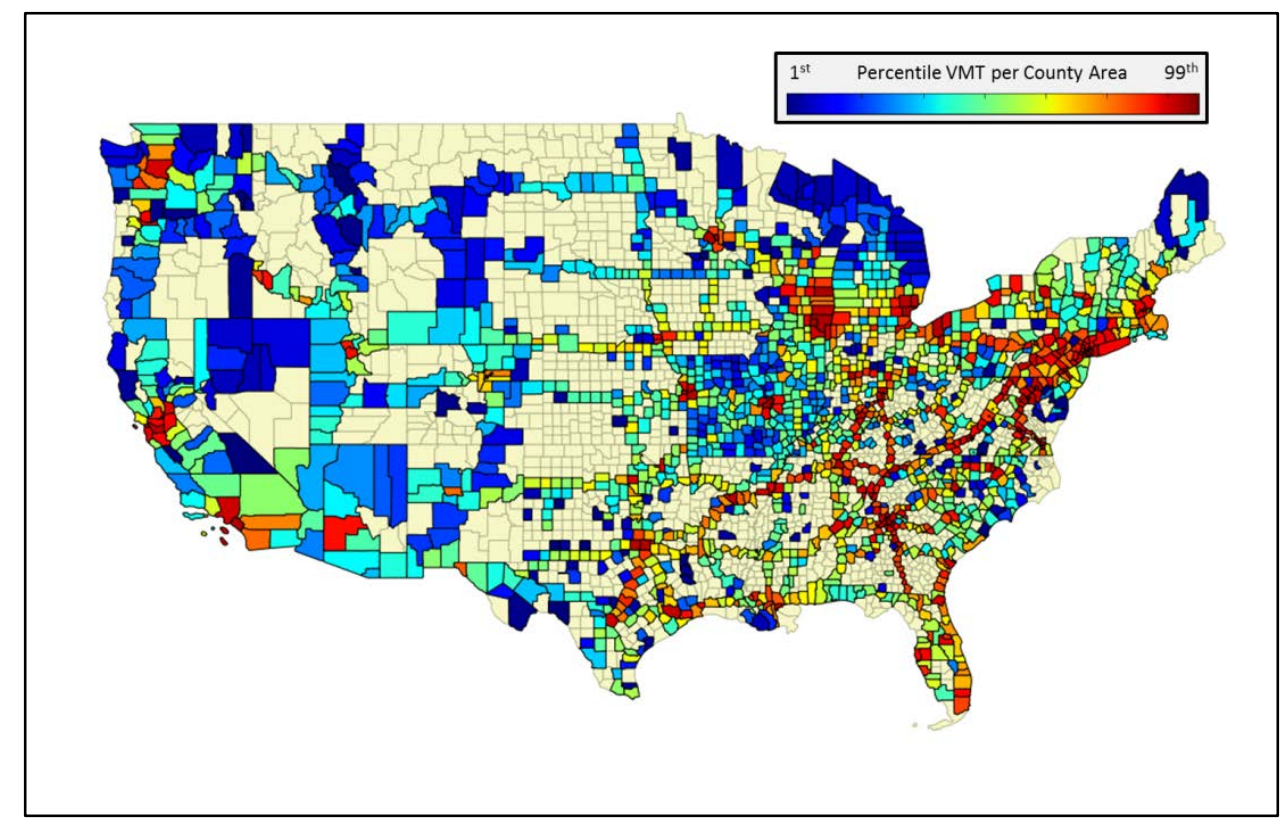

Figure 24. EPA MOVES VMT database mapped by county for MD/HD trucks operating on controlled access roads

Because VMT from the MOVES database only allows resolution at the county level, VMT must be subdivided within each county prior to applying activity weighting. For the analysis of road grade by distance, county MD/HD controlled access VMT are uniformly distributed over queried grade points in a county (points uniformly spaced at 100 points per mile on all controlled access roads within each county). For half hill statistics, weights are generated for every half hill according to Equation 3.

$$
w_{i}=V M T_{\text {county }, i} *\left(\frac{d_{i}}{\sum_{i=1}^{h_{\text {county }, i}} d_{i}}\right)
$$

where $\mathrm{w}_{\mathrm{i}}$ is the individual weight of each half hill, $\mathrm{VMT}_{\text {county, }}$ is the MOVES VMT from the county containing half hill $\mathrm{i}, \mathrm{d}_{\mathrm{i}}$ is the distance of half hill $\mathrm{i}$, and $\mathrm{hh}_{\text {county, }} \mathrm{i}$ is the total count of all half hills from the county in question. These weights are then used to generate activity-weight distributions of half hill distance as shown in Equation 4.

$$
P(d)=\left(\sum_{i=1}^{n}\left\{\begin{array}{cc}
d_{i}<d & w_{i} \\
\text { else } & 0
\end{array}\right) / \sum_{i=1}^{n} w_{i}\right.
$$


Figure 25 provides activity-weighted distributions of grade by distance for the five truck speed limits in question.

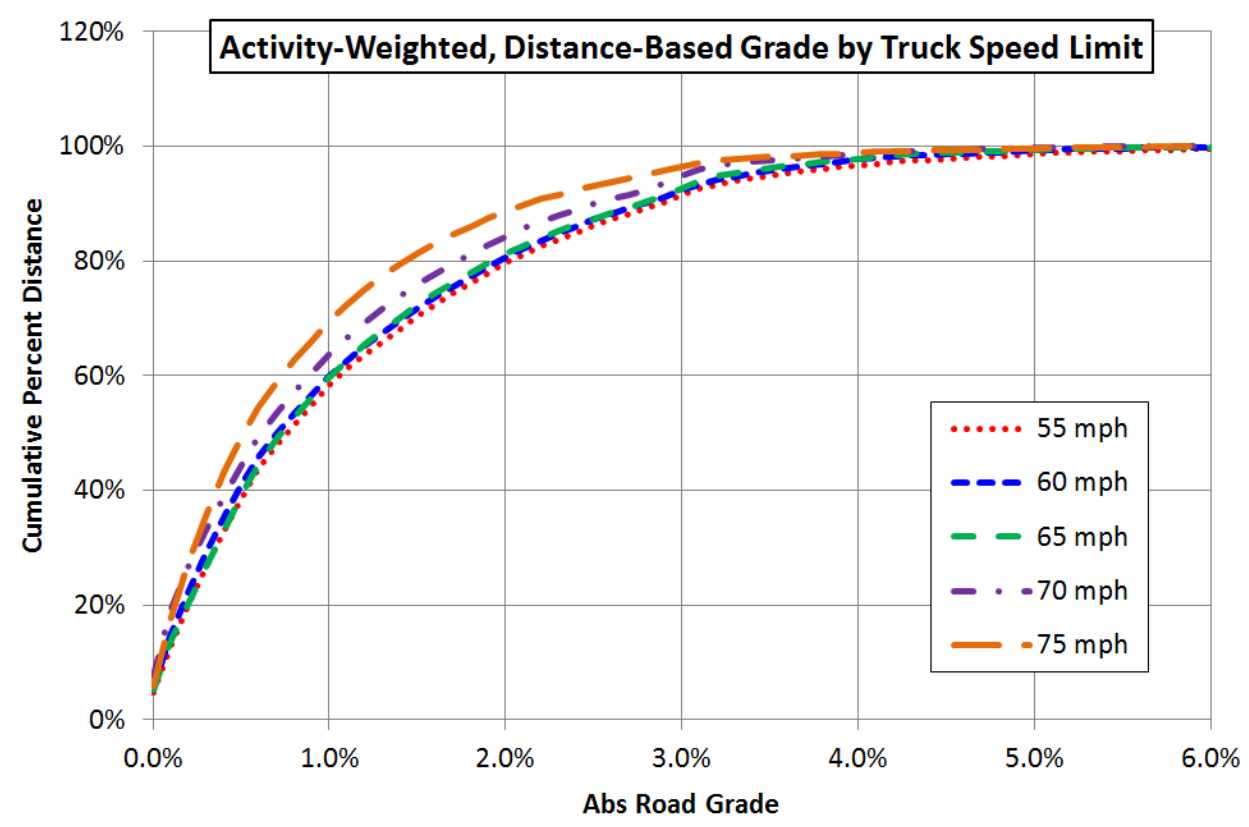

Figure 25. Activity-weighted, distance-based cumulative distributions of absolute road grade by truck speed limit

Similarly, activity weights are applied to the distance-based distributions of half hill distance by truck speed limit in Figure 26.

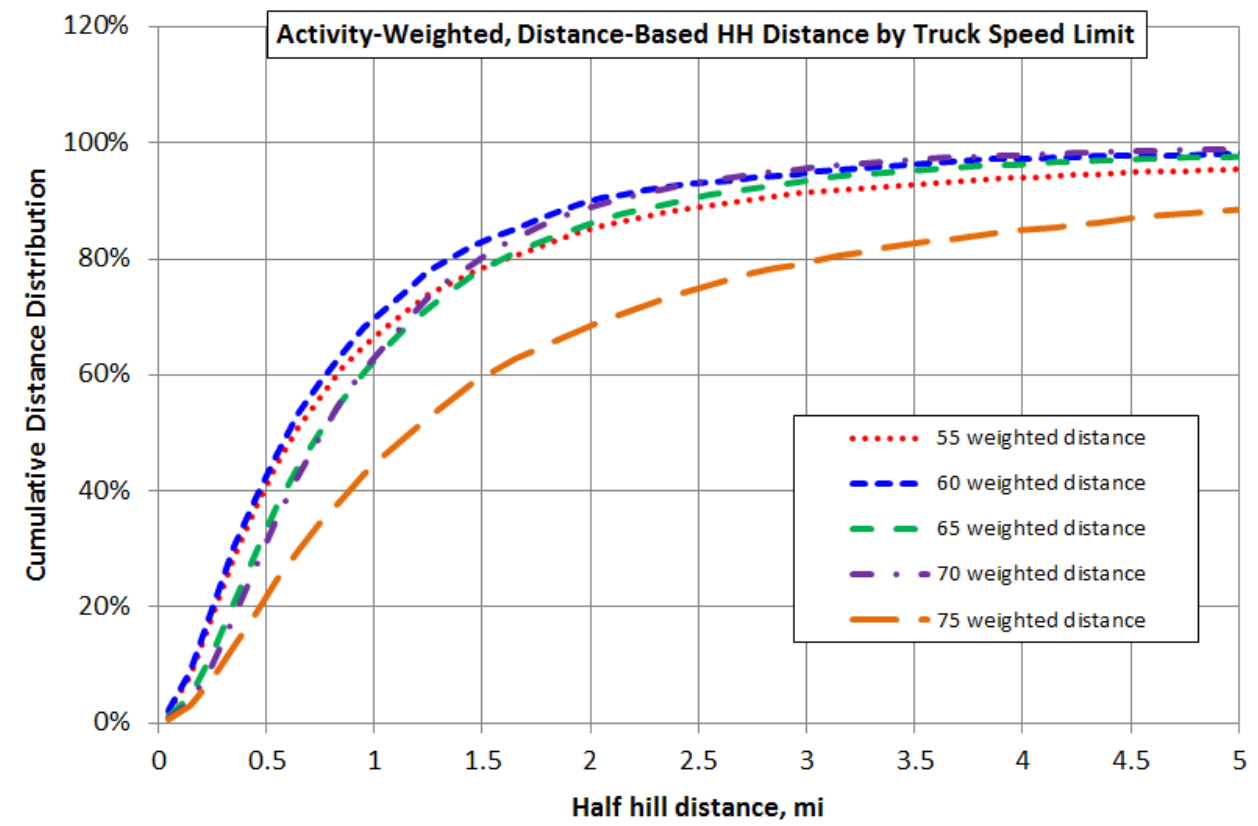

Figure 26. Activity-weighted, distance-based cumulative distributions of half hill distance by truck speed limit 
To align with existing EPA highway test cycle speeds at 55- and 65-mph, the TomTom data from roads with truck speed limits of 55- and 60-mph are grouped into a shared distribution to inform testing at 55-mph. The remaining truck speed limit data for of 65-, 70-, and 75-mph are grouped into a shared distribution to inform testing at $65-\mathrm{mph}$. Further future on-road data analysis may help evaluate how closely the fixed 55- and 65 -mph test cycle speeds align with actual on-road speeds for each of these road speed limit categories.

With the data now in two groups for EPA test cycles at 55- and 65-mph, the raw and activityweighted distributions of road grade by distance for both 55- and $65-\mathrm{mph}$ test groups are plotted in Figure 27. Activity-weighted distributions for both test groups closely match the raw data.

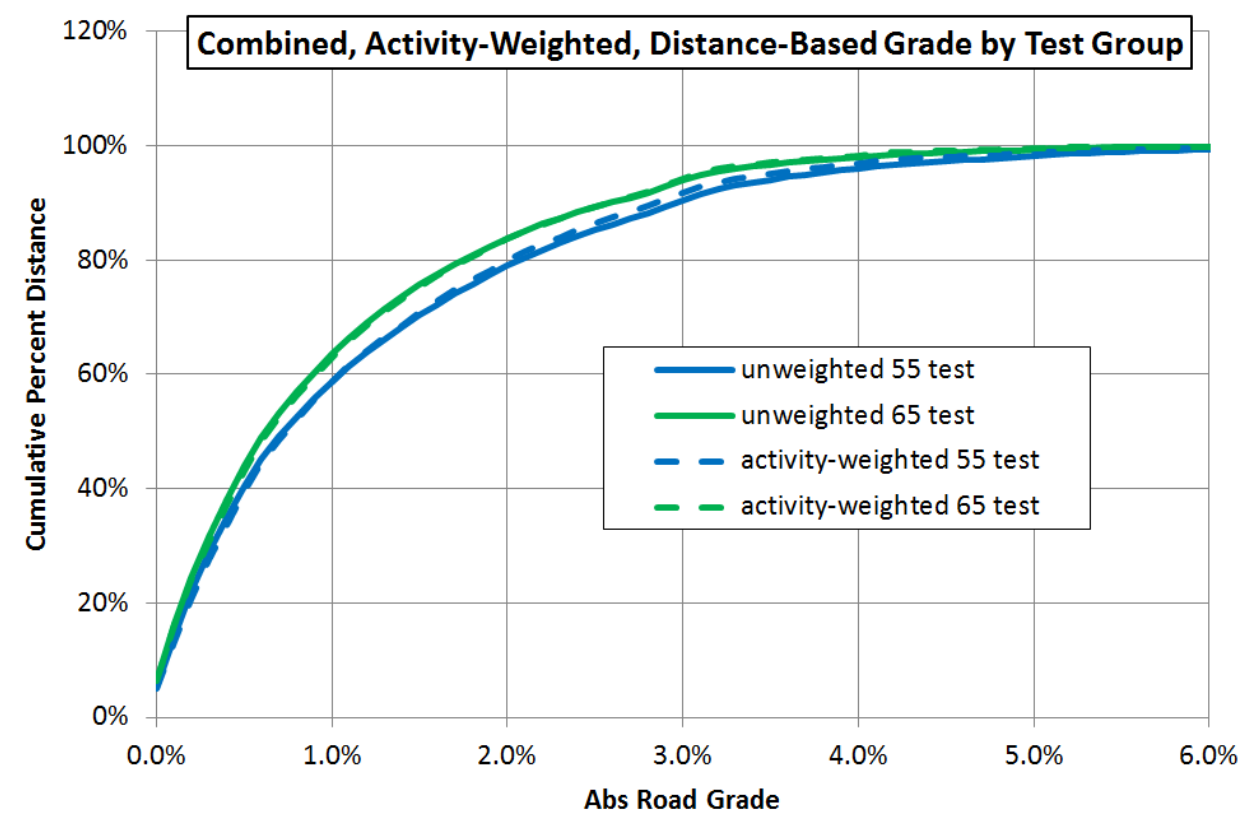

Figure 27. Combined activity-weighted, distance-based cumulative distributions of absolute road grade by target test speed

In order to demonstrate the statistical difference between activity-weighted 55-mph and 65-mph test groups, Kolmogorov-Smirnov (KS) hypothesis testing (as described in Appendix Section A.3) was conducted at a $95 \%$ confidence level. Hypothesis testing revealed a KS statistic of 0.046 and a P-value of 0.000 , indicating that the two distributions were determined to be statistically unique (i.e., the null hypothesis that the distributions are equivalent was rejected). 
Activity-weighted data are also generated for the distance-based distributions of half hill distances, shown in Figure 28. The application of activity weights shifts both the 55- and 65-mph distributions toward shorter half hill distances.

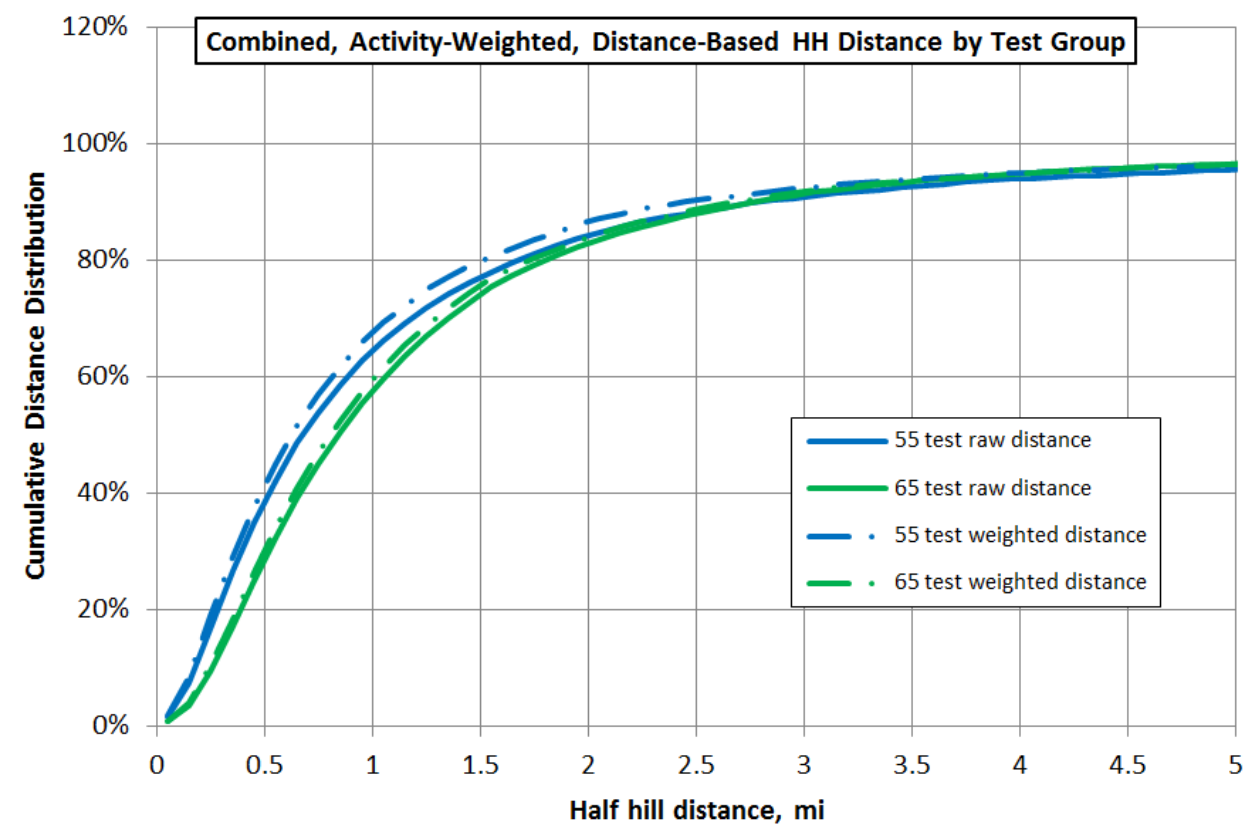

Figure 28. Combined activity-weighted, distance-based cumulative distributions of half hill distance by target test speed

In order to demonstrate the statistical difference between activity-weighted 55-mph and 65-mph test groups, KS hypothesis testing (as described in Appendix Section A.3) was conducted at a $95 \%$ confidence level. Hypothesis testing revealed a KS statistic of 0.111 and a P-value of 0.000 indicating that the two distributions were determined to be statistically unique (i.e., the null hypothesis that the distributions are equivalent was rejected).

The combined activity-weighted, distance-based cumulative distributions of road grade and half hill distance shown in Figures 27 and 28 will serve as the basis in Section 4 for evaluating the representativeness of sample road grade profiles from synthetic generation and identification of local routes.

Figures 27 and 28 show that application of activity weights has relatively little impact on the underlying raw distributions. An explanation of this outcome is provided in Appendix Section A.4. Please refer to Appendix Section A.5 for a discussion on why grade by distance distributions are used as the national target in this analysis (as opposed to average half hill grade distributions). Supplementary data distributions from the half hill analysis not used for determining representativeness of local grade profiles are included in Appendix B. 


\section{Nationally Representative Sample Grade Profiles}

Two procedures for generating road grade profiles representative of the previously presented national statistics for testing and simulation purposes are implemented. The first method utilizes random number generation in a Monte Carlo process to synthesize a large number of profiles from which the most representative single profile is selected. The second involves data mining the TomTom national road grade database for a continuous section of actual highway that has statistics matching the national distributions within a predefined tolerance.

The statistical representativeness of sample grade profiles will be evaluated against the national, distance-based, activity-weighted, cumulative distributions of absolute road grade and half hill distance for both the 55-mph and 65-mph test groups using KS hypothesis testing (as described in Appendix Section A.3). KS hypothesis testing will be conducted at a 95\% confidence level and we will target sample profiles with KS statistics of no greater than 0.05 and 0.10 for absolute road grade and half hill distance, respectively.

The sample grade profiles developed in this section of the report are intended to be paired with constant speed drive cycles at 55- and 65-mph and potentially used in the EPA's Greenhouse Gas Emissions Model certification tool as well as in dynamometer testing of medium- and heavy duty vehicles and their powertrains.

\subsection{Synthetic Routes}

As part of this project, NREL developed an extension of its DRIVE tool [9] to synthesize representative road grade profiles from statistical distributions of the activity-weighted national road network. This extension builds on DRIVE's core capability of generating representative drive cycles from large on-road datasets of real-world driving (see [27] for an example of compressing a large, real-world, drive cycle dataset into a single, statistically representative cycle).

Experience with DRIVE was leveraged to construct a method for synthesizing statistically representative road grade profiles. This procedure employs a Monte Carlo method in which grade profiles are synthesized one half hill at a time. Characteristics for the first half hill are determined by randomly sampling national frequency-based distributions of half hill distance, average grade, and maximum to average grade ratio (plots of frequency-based distribution are included in Appendix Section B). Once distance, $d_{i}$, average grade, $g_{i}$, and grade ratio, $r_{i}$, characteristics have been determined, the shape of the first half hill is constructed using the template shown in Figure 29. 


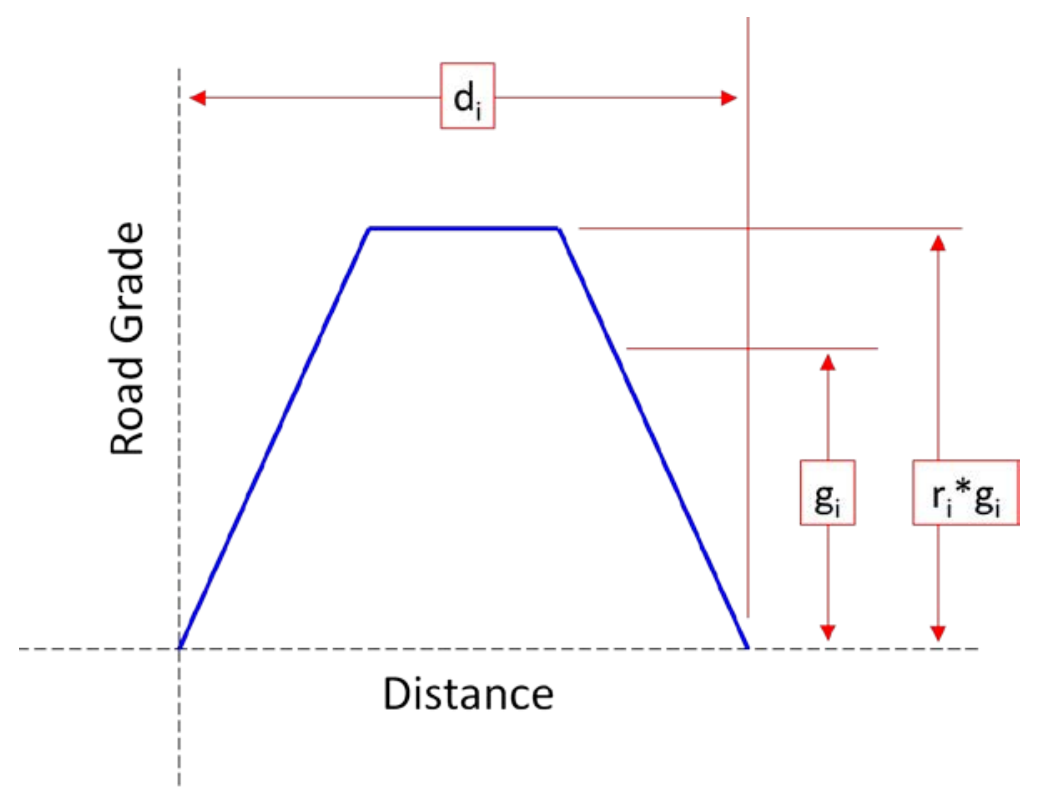

Figure 29. Synthetic grade profile template

This template assumes half hill road grades in the shape of a trapezoid (reduces to an isosceles triangle in the case $r_{i} \geq 2$ ) vertically symmetrical about $d_{i} / 2$. The vertical coordinates for this template are readily determined by evaluating maximum grade as the product of the grade ratio and average grade. Horizontal coordinates are determined geometrically as a function of half hill distance and grade ratio.

This process is repeated with half hill grade sign calculated such that the profile descends when above starting elevation and ascends when below starting elevation. Half hills are appended to the synthetic profile until a target distance is reached, at which point one additional half hill is appended with average grade calculated to ensure net zero elevation change over the entire sample profile. After the synthetic grade profile is complete, relative elevation is calculated by integrating grade with respect to distance.

This Monte Carlo approach for generating synthetic road grade profiles is computationally efficient, which enables the generation of millions of potential sample profiles. A full design of experiments was conducted with this method for both the 55-mph and 65-mph test groups. For each test group, sample profiles were generated at target profile distances from 3 to 30 miles in 3 -mile increments. For each combination of test group and target profile distance, a fixed number of sample profiles were generated. The most representative profile from each combination was saved for further analysis. The degree to which sample profiles are representative of national distributions was quantified by calculating the weighted average of KS statistics for absolute grade and half hill distance with the KS statistic for absolute grade weighted 2:1, respectively (reflecting a greater emphasis on matching the distribution of absolute road grade). The fixed number of sample profiles was swept from 1,000 to 1,000,000 sample profiles in logarithmic intervals of base ten. 


\subsubsection{5-mph Test Group}

The results of this design of experiments for the 55-mph test group in terms of the KS statistic for road grade match are shown in Figure 30. The fixed black line at a KS statistic of 0.05 represents the previously stated goal (KS statistics close to zero indicate better matches to the national dataset). The first trend observed from this plot is the tendency for synthetic profiles of greater distance to exhibit lower KS statistics. This result is intuitive from the standpoint that longer target profile distances allow for sample distributions of road grade to be more evenly distributed (i.e., there is not enough opportunity in the 3-mile sample profiles to capture all of the grade variation observed in the national dataset).

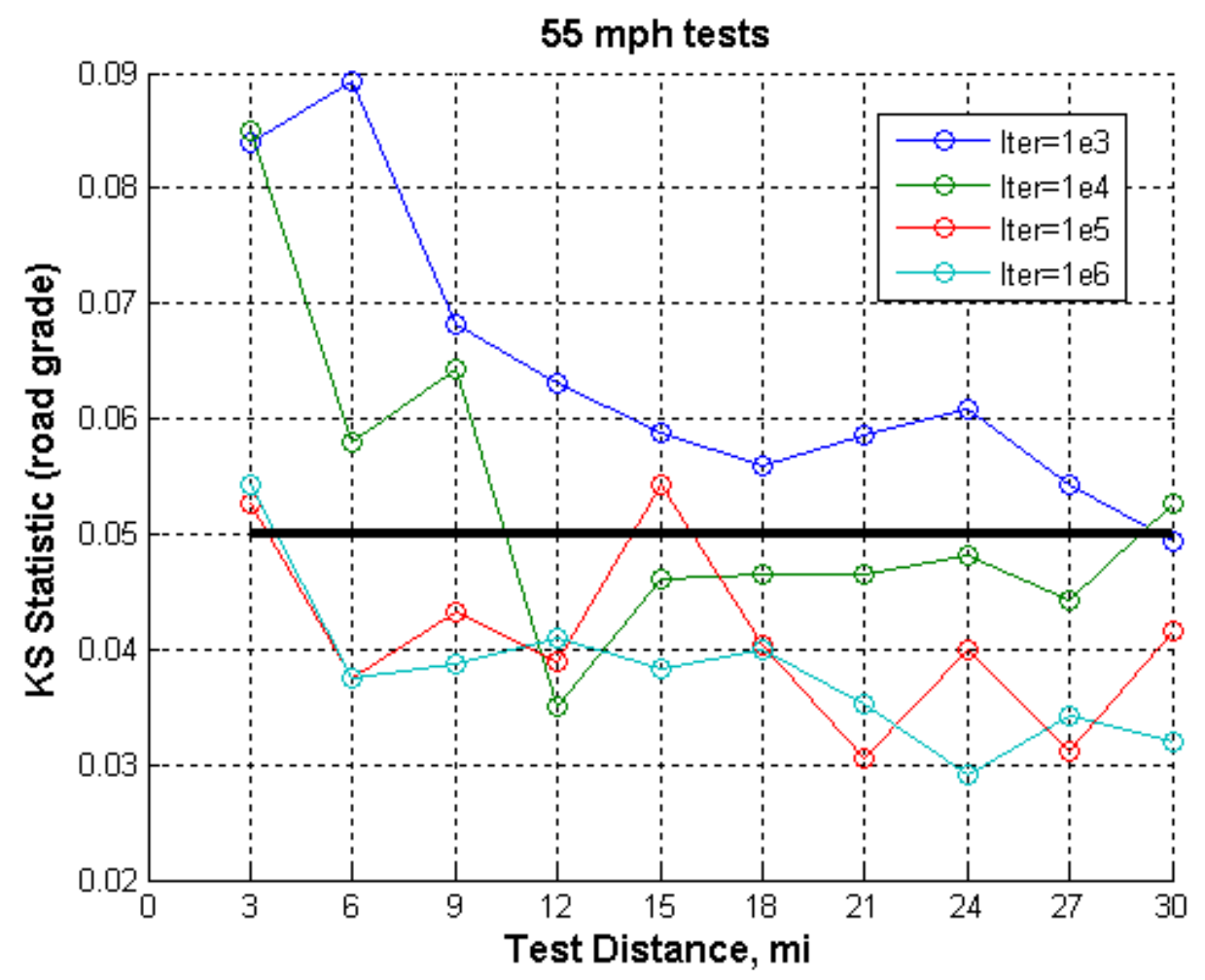

Figure 30. KS statistics for absolute road grade match in the 55-mph test group with respect to target profile distance and maximum iteration count

Secondly, KS statistics are (in general) improved by allowing a larger number of fixed iterations from which to draw. This finding is consistent with other Monte Carlo tools that (in general) experience improved performance given larger sample sizes. One exception to this trend is observed at the 12-mile test distance where the top scoring profile from the pool of 10,000 samples had a lower KS statistic than the top scoring profiles from the pools of 100,000 and $1,000,000$ samples. This exception demonstrates the uncertainty in convergence time associated with random number sampling. 
In addition to sample profiles matching the national dataset in terms of absolute road grades, we would like to identify profiles with strong matches in terms of half hill distances. Design of experiment results for the 55-mph test group in terms of KS statistic for half hill distance match are shown in Figure 31. The fixed black line at a KS statistic of 0.10 represents the previously stated goal. As with the plot of KS statistics for road grade match, there are similar trends with respect to test profile distance and number of iterations.

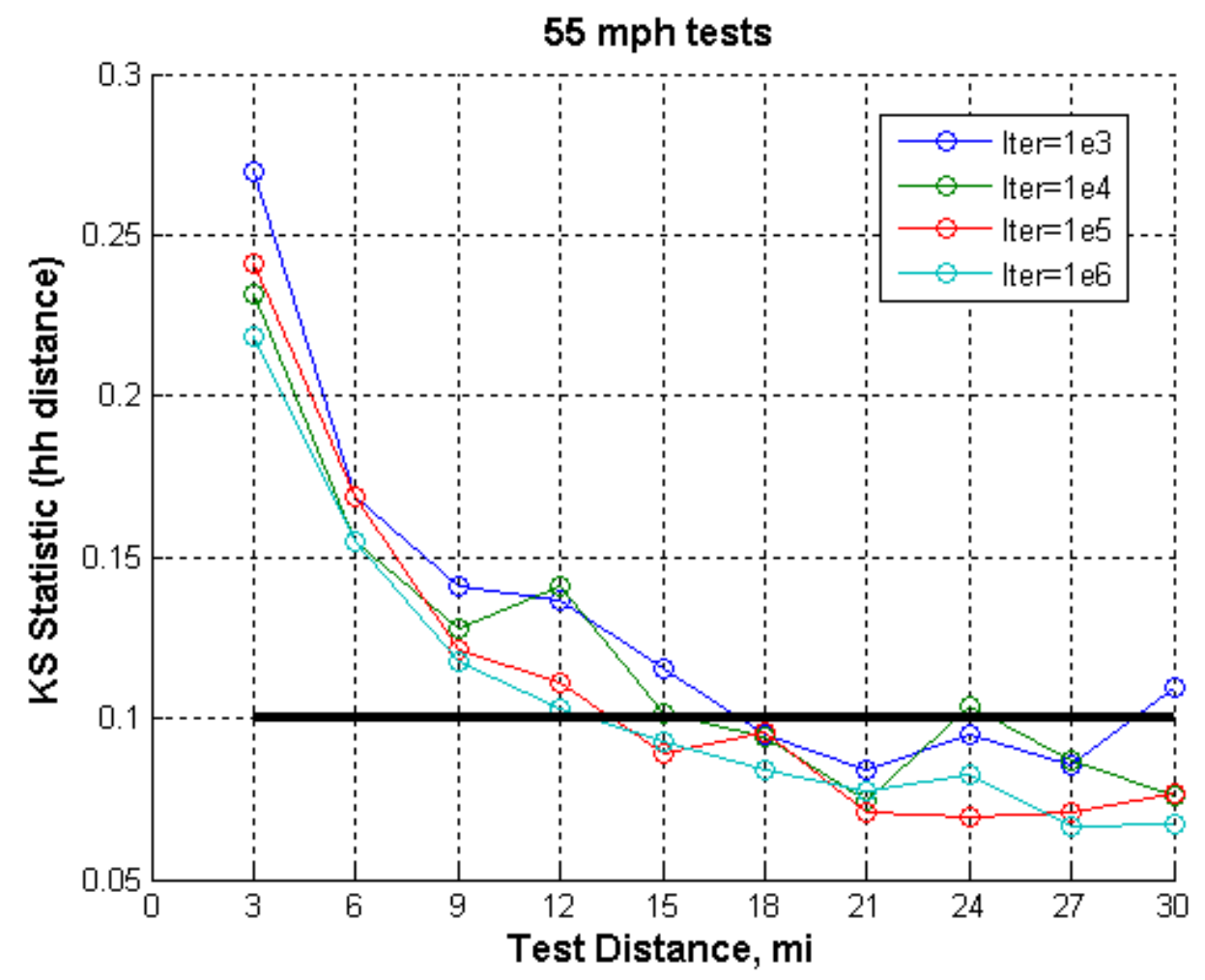

Figure 31. KS statistics for half hill distance match in the 55-mph test group with respect to target profile distance and maximum iteration count

Having mapped the performance of the synthetic profile generator, we are now interested in identifying a small number of sample profiles for closer examination. The sample profiles of greatest interest are those most closely matching the national dataset (low weighted KS statistic) and those of shortest distance. The objective to identify sample profiles of short distance is driven by the desire to minimize the significant time and cost requirements associated with dynamometer testing of vehicles and powertrains. As such, we will now present two sample profiles from the 12-mile profile distance target where the KS statistics for both road grade and half hill distance are below the stated goals of 0.05 and 0.10 , respectively. 
The first proposed road grade profile for 55-mph testing is shown in Figure 32 and named "150508 55mph 12mi 201210ofle6iter" (name denotes date of generation, target test speed, target distance, and iteration count). The red dashed line in the elevation subplot shows that the profile is net zero elevation.
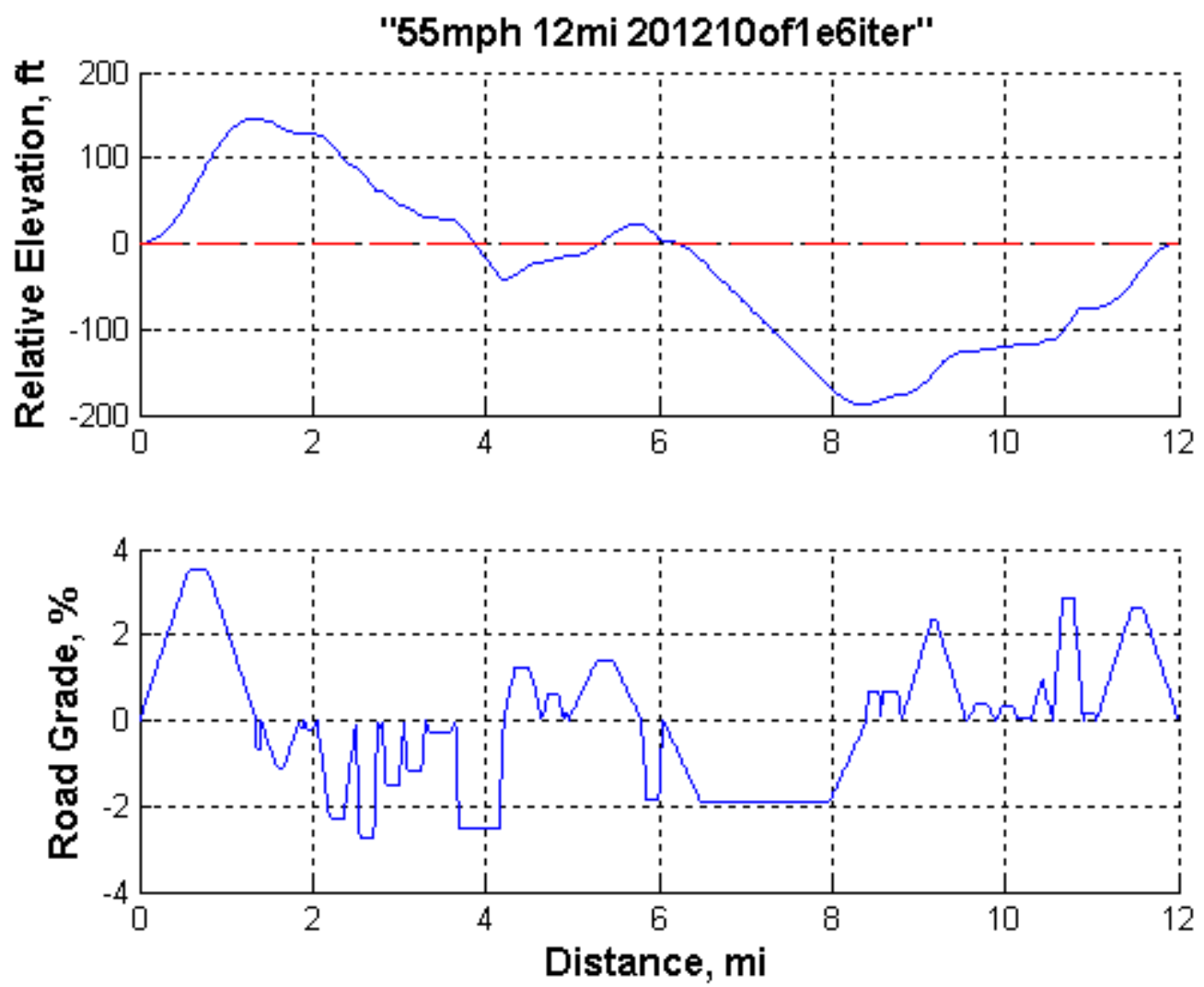

Figure 32. Elevation and road grade profile from sample "150508 55mph 12mi 201210 of1e6iter"

Red dashed line on elevation subplot shows net zero elevation change.

Distance-based cumulative distributions of absolute road grade and half hill distance for sample profile "150508 55mph 12mi 201210ofle6iter" are shown in Figure 33. National activityweighted, cumulative distributions of the same parameters are provided as reference for the degree of match to the national dataset. Recalling that the KS statistic is a measure of similarity between two distributions and that a KS statistic of $<0.05$ was targeted for the distribution of absolute road grade, this sample achieved a KS statistic of 0.049 and a P-value of 0.316 and failed to reject the null hypothesis that the distributions are equivalent at a $95 \%$ confidence level. Recalling that a KS statistic of $<0.10$ was targeted for the distribution of half hill distance, this sample achieved a KS statistic of 0.095 and a P-value of 0.603 and failed to reject the null hypothesis that the distributions are equivalent at a $95 \%$ confidence level. 

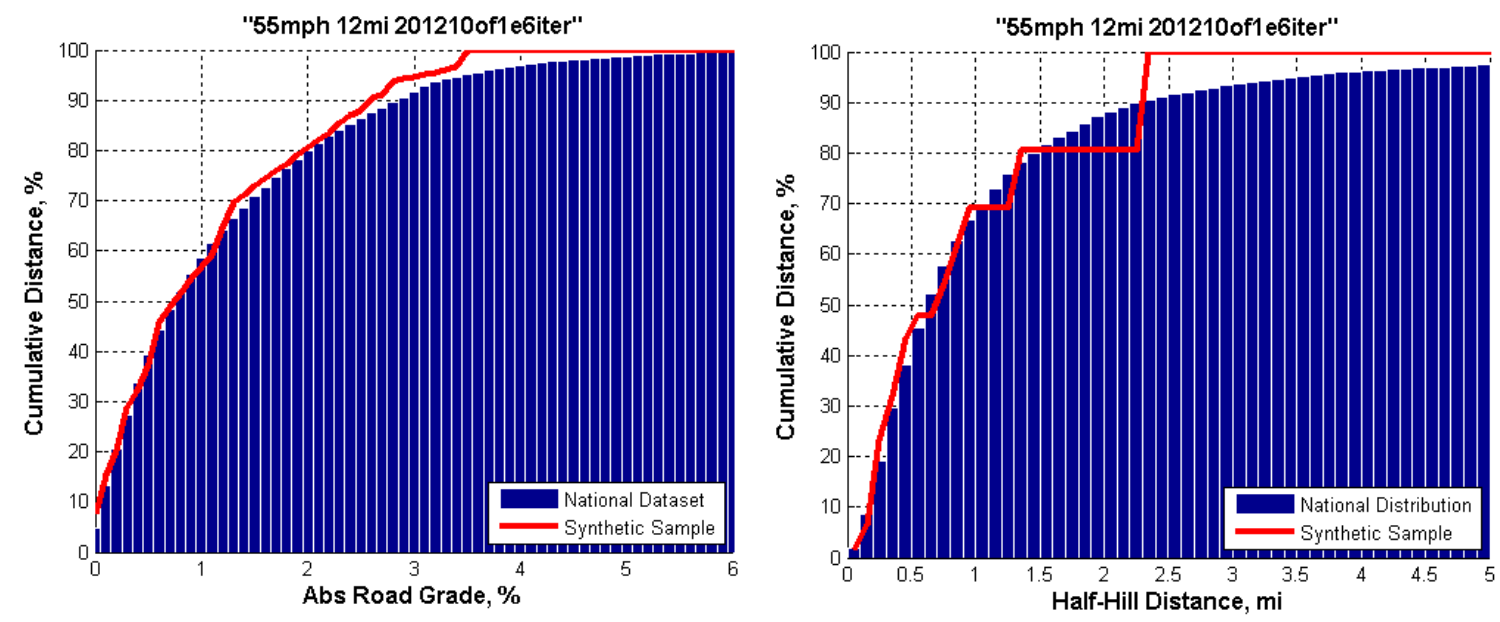

Figure 33. Comparison of distance-based cumulative distributions between national activityweighted dataset and sample profile " $15050855 \mathrm{mph} 12 \mathrm{mi} 201210$ of $1 \mathrm{e} 6$ iter" in terms of absolute road grade (left) and half hill distance (right)

The second proposed road grade profile for 55-mph testing is named " $15050855 \mathrm{mph} 12 \mathrm{mi}$ 727695of1 e6iter" (name denotes date of generation, target test speed, target distance, and iteration count) and is shown in Figure 34. The red dashed line in the elevation subplot shows that the profile is net zero elevation.
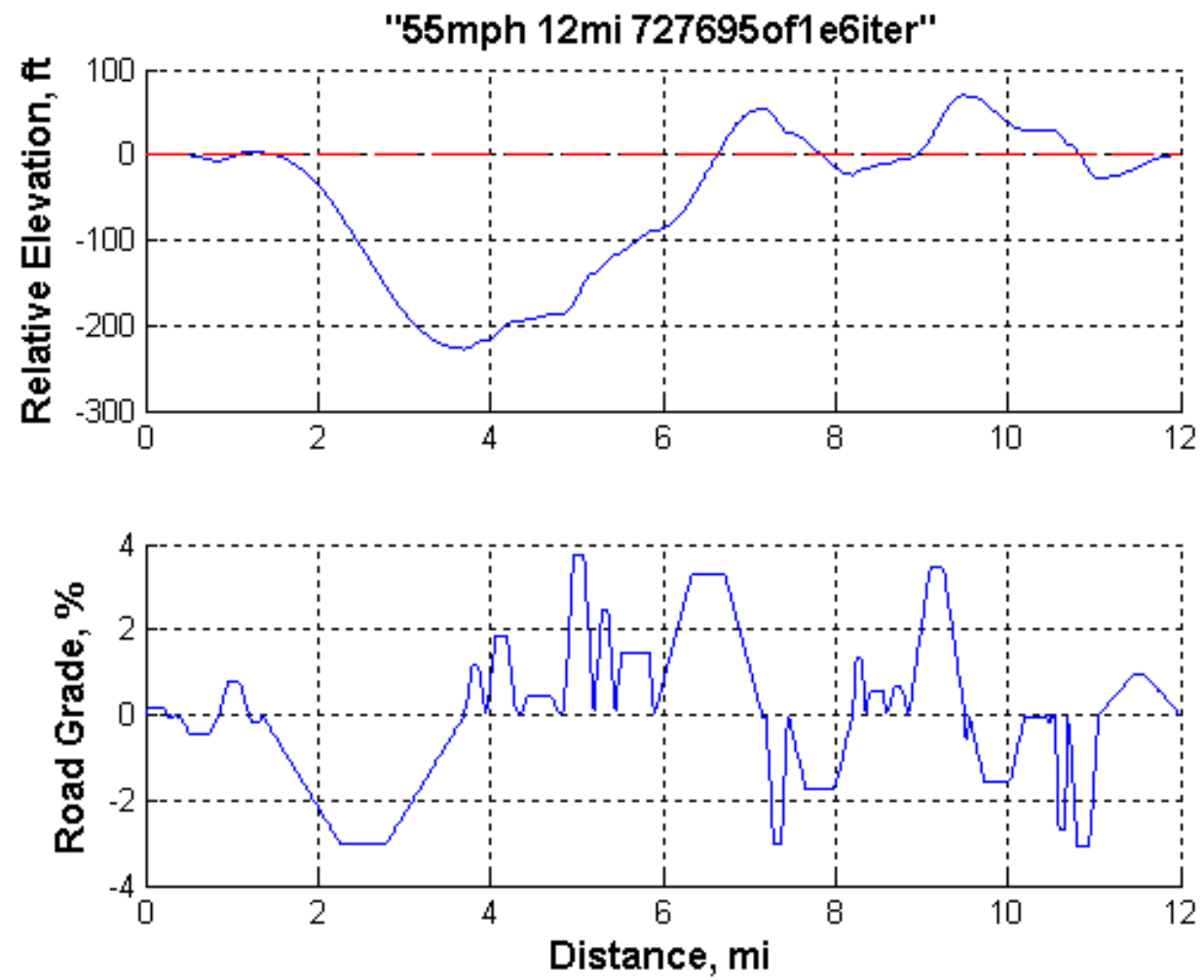

Figure 34. Elevation and road grade profile from sample "150508 55mph 12mi 727695of1e6iter"

Red dashed line on the elevation subplot is provided to show net zero elevation change. 
Distance-based, cumulative distributions of absolute road grade and half hill distance for sample profile " 150508 55mph 12mi 727695ofle6iter" are shown in Figure 35. National activityweighted, cumulative distributions of the same parameters are provided as reference for the degree of match to the national dataset. Recalling that the KS statistic is a measure of similarity between two distributions and that a KS statistic of $<0.05$ was targeted for the distribution of absolute road grade, this sample achieved a KS statistic of 0.047 and a P-value of 0.346 and failed to reject the null hypothesis that the distributions are equivalent at a $95 \%$ confidence level. Recalling that a KS statistic of $<0.10$ was targeted for the distribution of half hill distance, this sample achieved a KS statistic of 0.095 and a P-value of 0.561 and failed to reject the null hypothesis that the distributions are equivalent at a $95 \%$ confidence level.
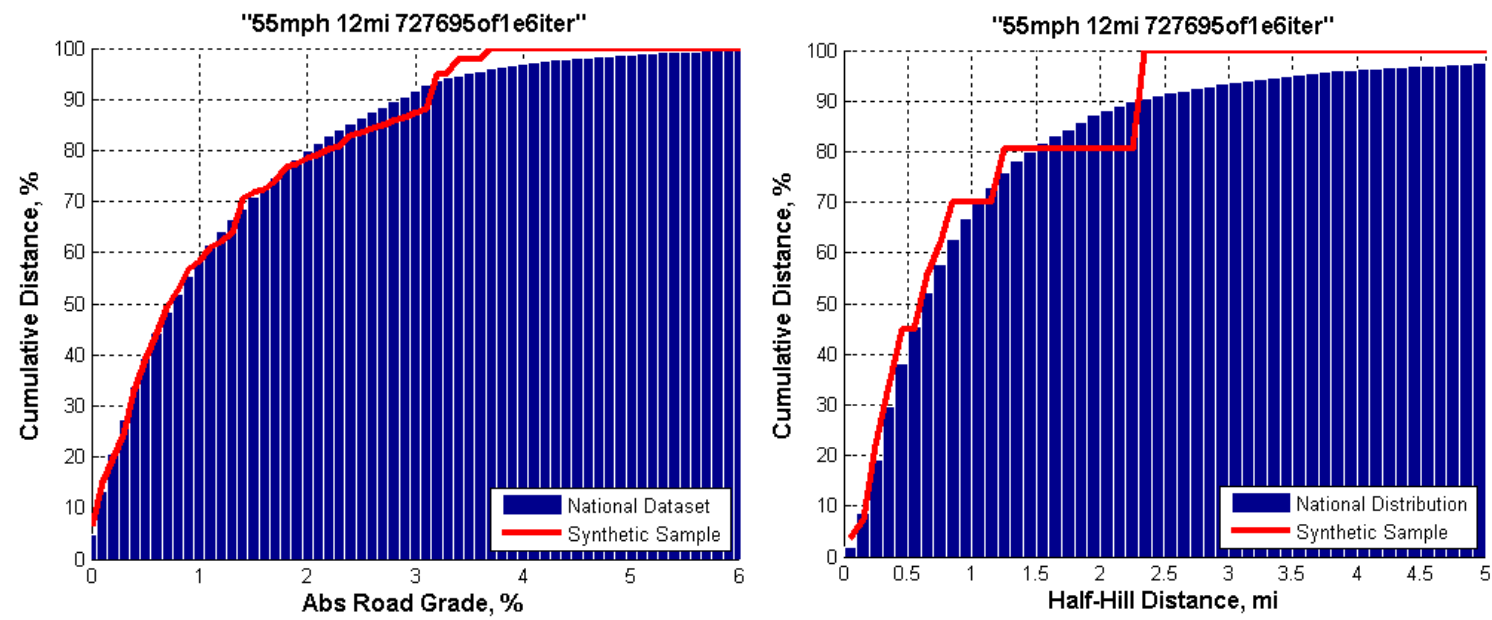

Figure 35. Comparison of distance-based cumulative distributions between a national activityweighted dataset and sample profile " $15050855 \mathrm{mph} 12 \mathrm{mi} 727695$ of1e6iter" in terms of absolute road grade (left) and half hill distance (right) 


\subsubsection{5-mph Test Group}

The results of the design of experiments for the 65-mph test group in terms of the KS statistic for road grade match are shown in Figure 36. A fixed black line at a KS statistic of 0.05 represents our previously stated goal (recall that KS statistics close to zero indicate better matches to the national dataset). As with the plots of KS statistics for the 55-mph group synthetic profiles, the trends are similar with respect to test profile distance and number of iterations.

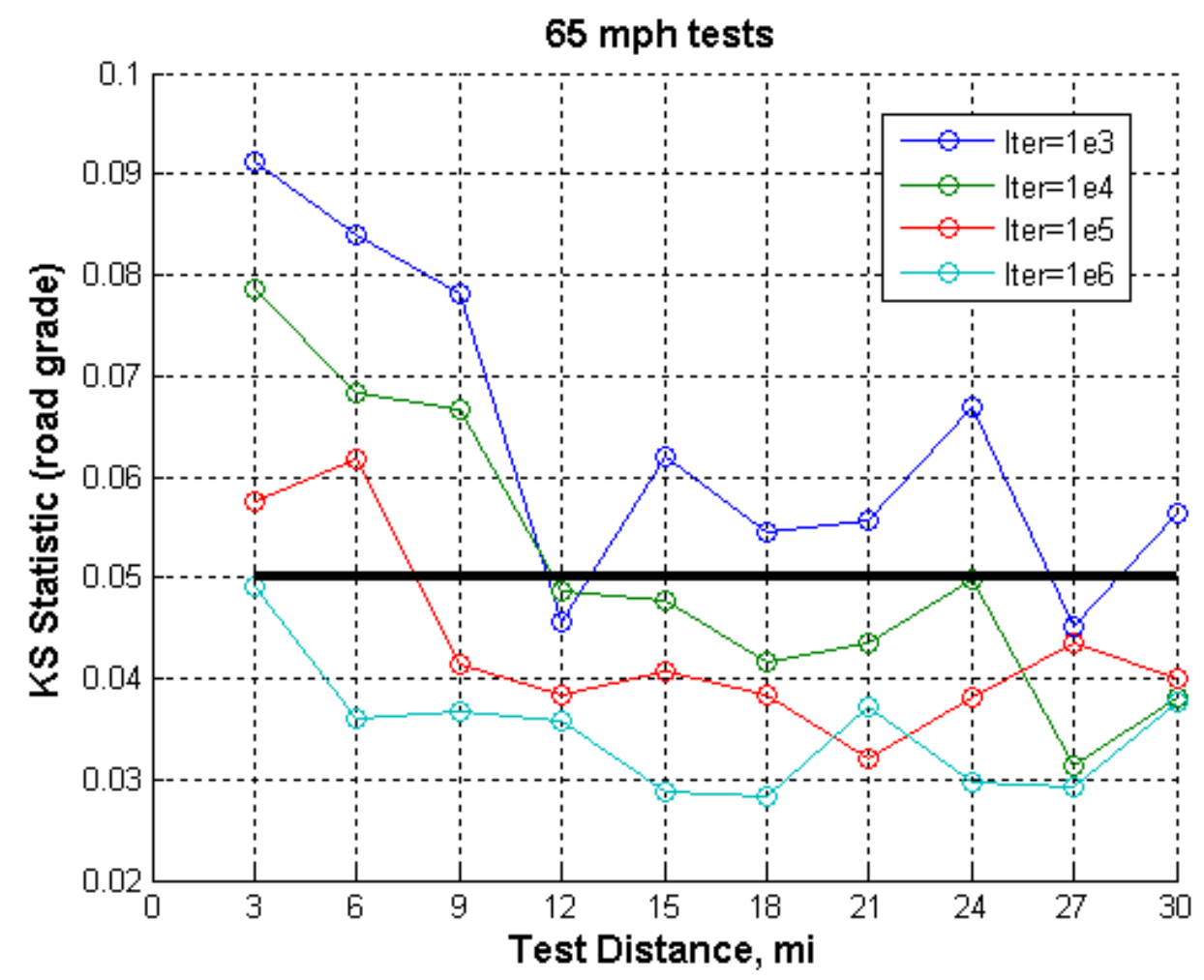

Figure 36. KS statistics for absolute road grade match in the 65-mph test group with respect to target profile distance and maximum iteration count 
Design-of-experiment results for the 65-mph test group in terms of the KS statistic for half hill distance match are shown in Figure 37. A fixed black line represents the goal of a KS statistic of 0.10 . As with the plot of KS statistics for road grade match, we see similar trends with respect to the test profile distance and number of iterations.

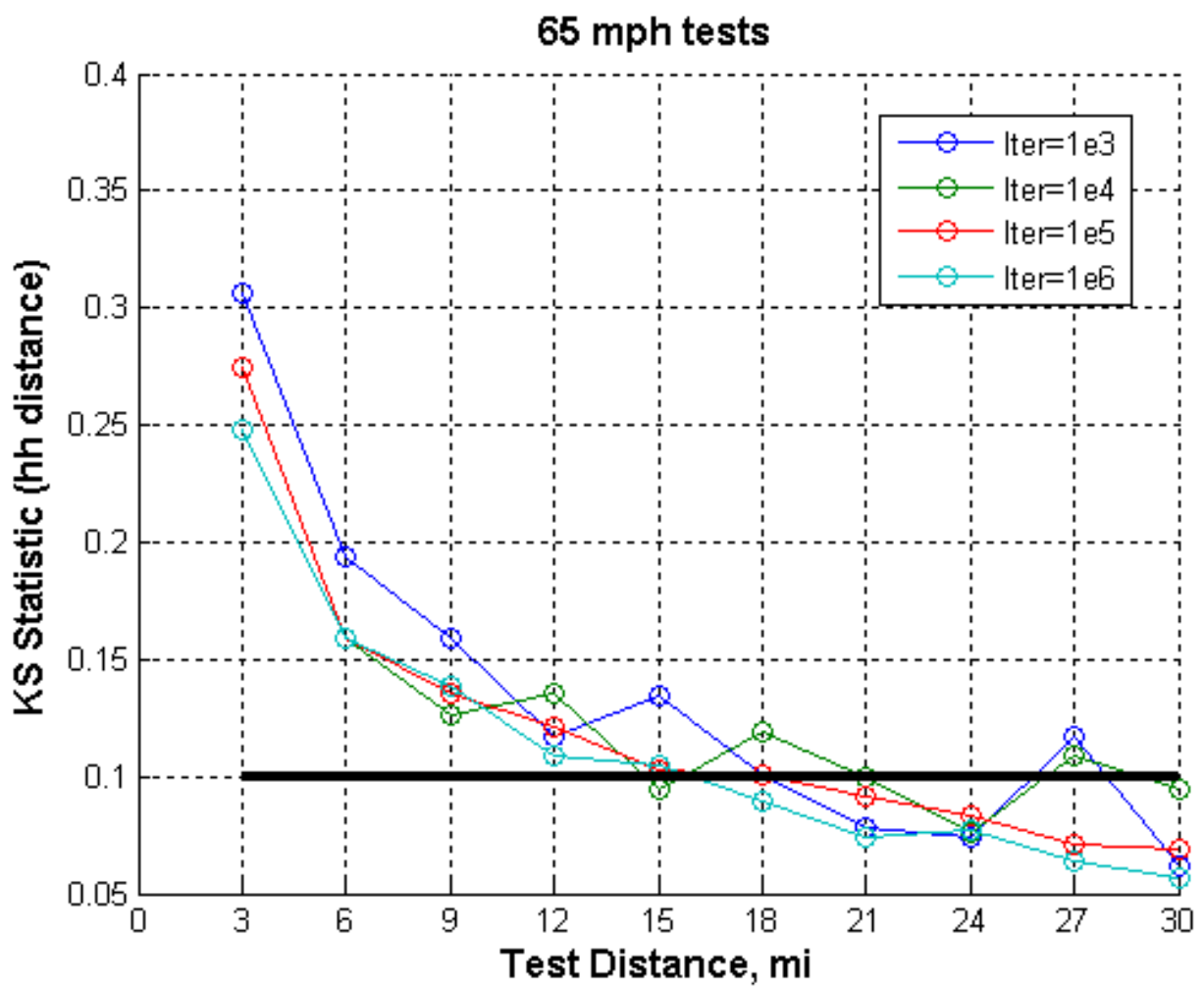

Figure 37. KS statistics for half hill distance match in the 65-mph test group with respect to target profile distance and maximum iteration count

Having mapped the performance of the synthetic profile generator, we are now interested in identifying a small number of sample profiles for closer examination. Sample profiles of greatest interest are those most closely matching the national dataset (low weighted KS statistic) and those of shortest distance. The objective to identify sample profiles of short distance is driven by the desire to minimize the significant time and cost requirements associated with dynamometer testing of vehicles and powertrains. Two sample profiles are presented for the 15-mile profile distance target where KS statistics for both road grade and half hill distance are below the goals of 0.05 and 0.10 , respectively. 
The first proposed road grade profile for 65 -mph testing, named " $15050865 \mathrm{mph} 15 \mathrm{mi}$ 467684ofle6iter" (name denotes date of generation, target test speed, target distance, and iteration count), is shown in Figure 38. The red dashed line in the elevation subplot shows that the profile is net zero elevation.
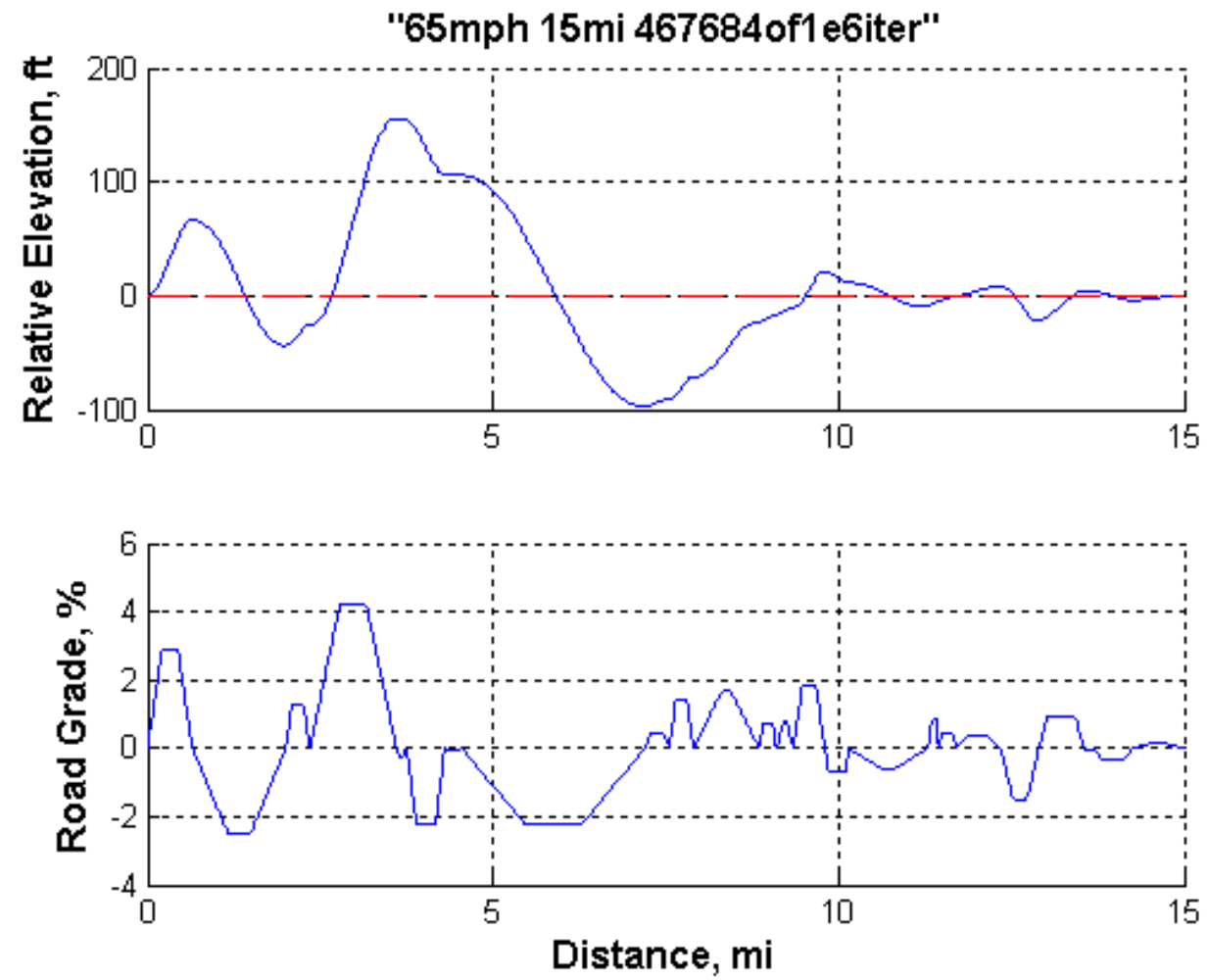

Figure 38. Elevation and road grade profile from sample "150508 65mph 15mi 467684of1e6iter"

Red dashed line on elevation subplot shows there is net zero elevation change.

Distance-based cumulative distributions of absolute road grade and half hill distance for sample profile " 150508 65mph 15mi 467684ofle6iter" are shown in Figure 39. National activityweighted cumulative distributions of the same parameters are provided as reference for the degree of match to the national dataset. Recalling that the KS statistic is a measure of similarity between two distributions and that a KS statistic of $<0.05$ was targeted for the distribution of absolute road grade, this sample achieved a KS statistic of 0.041 and a P-value of 0.365 and failed to reject the null hypothesis that the distributions are equivalent at a $95 \%$ confidence level. Recalling that a KS statistic of $<0.10$ was targeted for the distribution of half hill distance, this sample achieved a KS statistic of 0.098 and a P-value of 0.631 and failed to reject the null hypothesis that the distributions are equivalent at a $95 \%$ confidence level. 

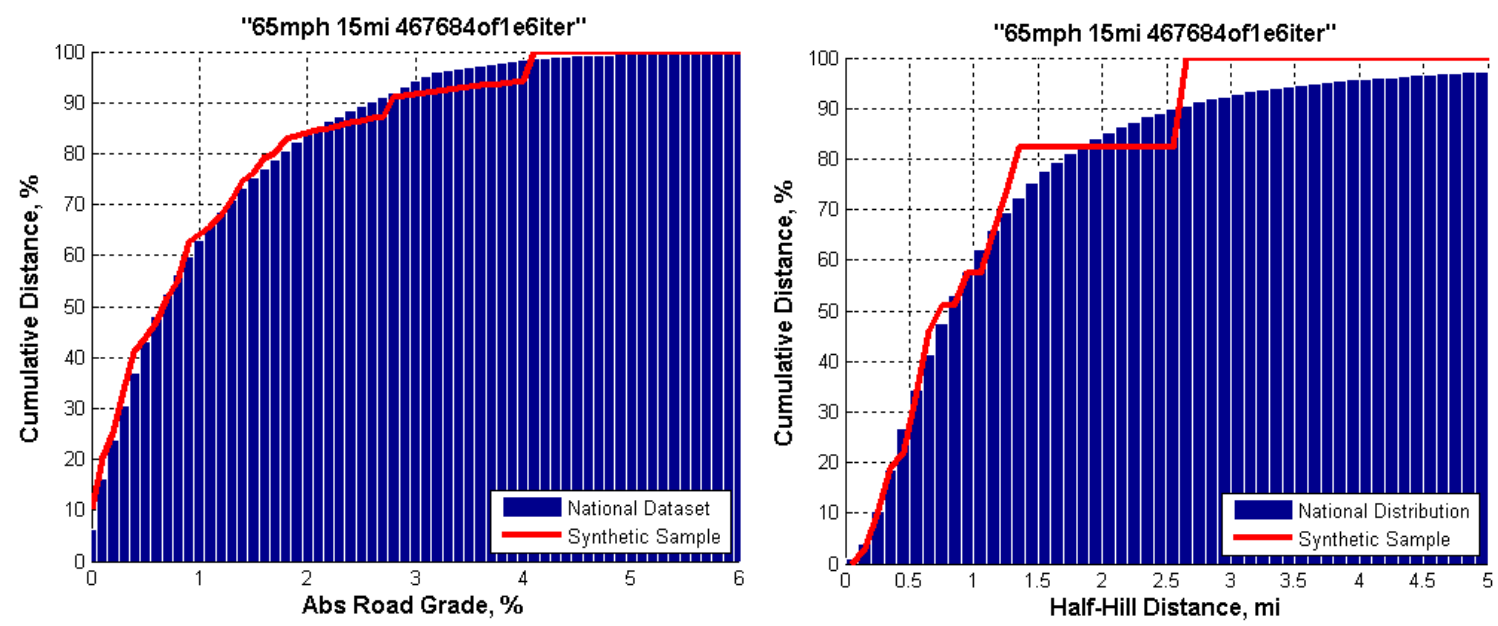

Figure 39. Comparison of distance-based, cumulative distributions between national activityweighted dataset and sample profile " $15050865 \mathrm{mph} 15 \mathrm{mi} 467684$ of 1 e6iter" in terms of absolute road grade (left) and half hill distance (right)

The second proposed road grade profile, named "150508 65mph 15mi 883894ofle6iter" (name denotes date of generation, target test speed, target distance, and iteration count), for 65-mph testing is shown in Figure 40. The red dashed line in the elevation subplot shows that the profile is net zero elevation.
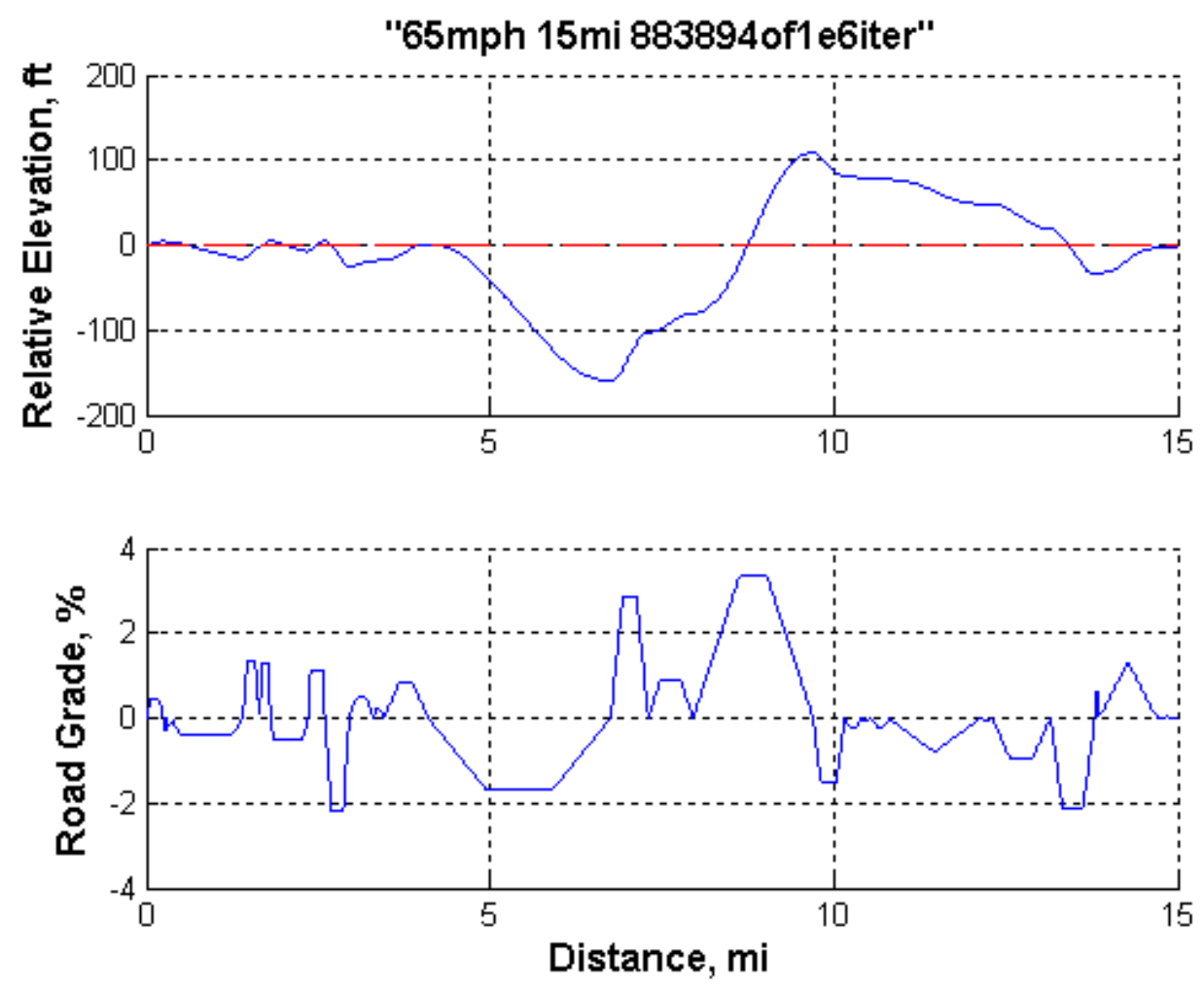

Figure 40. Elevation and road grade profile from sample "150508 65mph 15mi 883894of1e6iter" Red dashed line on elevation subplot shows net zero elevation change. 
Distance-based cumulative distributions of absolute road grade and half hill distance for sample profile "150508 65mph 15mi 883894ofle6iter" are shown in Figure 41. National activityweighted cumulative distributions of the same parameters are provided as reference for the degree of match to the national dataset. Recalling that the KS statistic is a measure of similarity between two distributions and that a KS statistic of $<0.05$ was targeted for the distribution of absolute road grade, this sample achieved a KS statistic of 0.047 and a P-value of 0.266 and failed to reject the null hypothesis that the distributions are equivalent at a $95 \%$ confidence level. Recalling that a KS statistic of $<0.10$ was targeted for the distribution of half hill distance, this sample achieved a KS statistic of 0.094 and a P-value of 0.621 and failed to reject the null hypothesis that the distributions are equivalent at a 95\% confidence level.
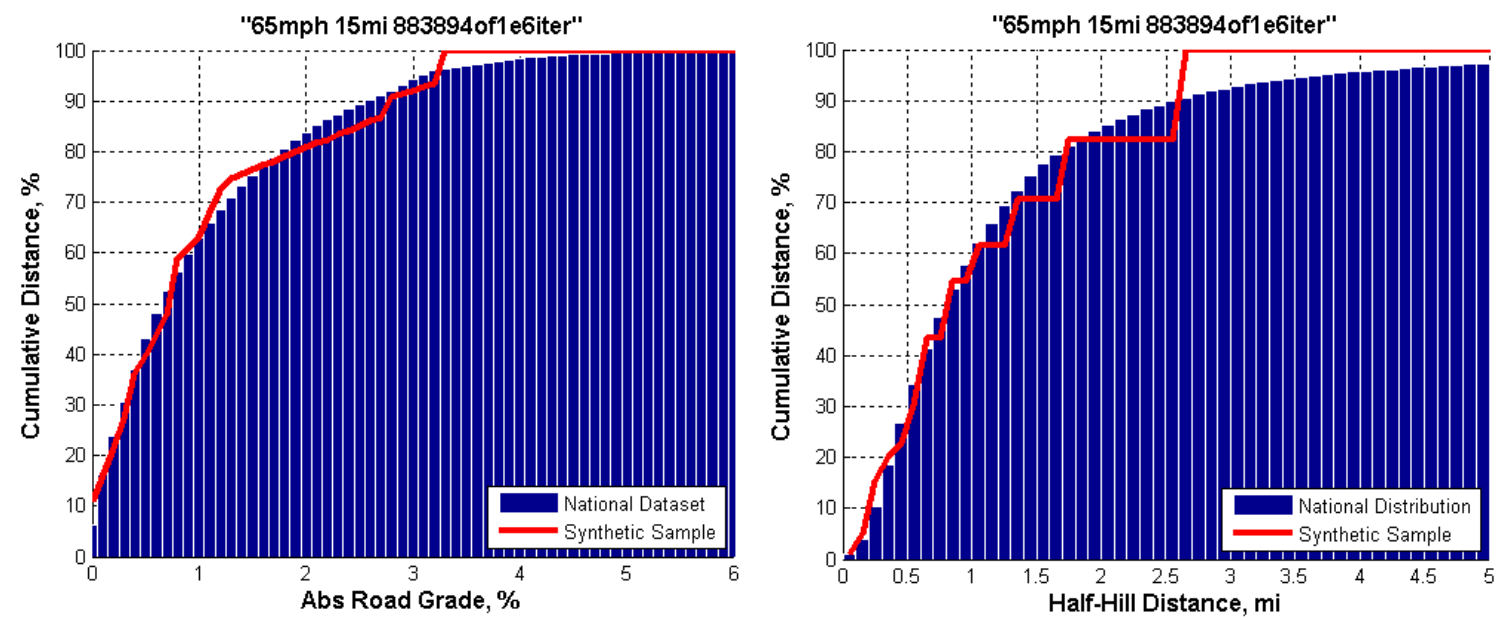

Figure 41. Comparison of distance-based cumulative distributions between national activityweighted dataset and sample profile " $15050865 \mathrm{mph} 15 \mathrm{mi} 883894$ of1e6iter" in terms of absolute road grade (left) and half hill distance (right)

\subsection{Local Routes}

As an alternative to generating synthetic grade profiles, the potential for extracting real-world grade profiles from continuous sections of highway using the TomTom national road grade database was explored. To this end, a procedure was established for processing the national network of controlled access highways and identifying stretches with statistics representative of the previously developed national activity-weighted distributions.

An example of this processing routine can be seen in Figure 42. For this example 13-mile stretch of highway, seven unique stretches of 10 miles or less are identified by starting at the beginning of the first half hill and expanding the search window as far as possible without exceeding the 10-mile limit. The 10-mile limit in this example is an input variable for the processing routine. This process is repeated from the beginning of the next sequential half hill until the end of the highway has been reached. For each unique stretch of highway, local distributions of grade by distance and half hill length are compared to the national activity-weighted distributions. A KS statistic is calculated for both the grade and distance distributions, and segments where both KS statistics are less than a predefined tolerance are recorded for further inspection. 


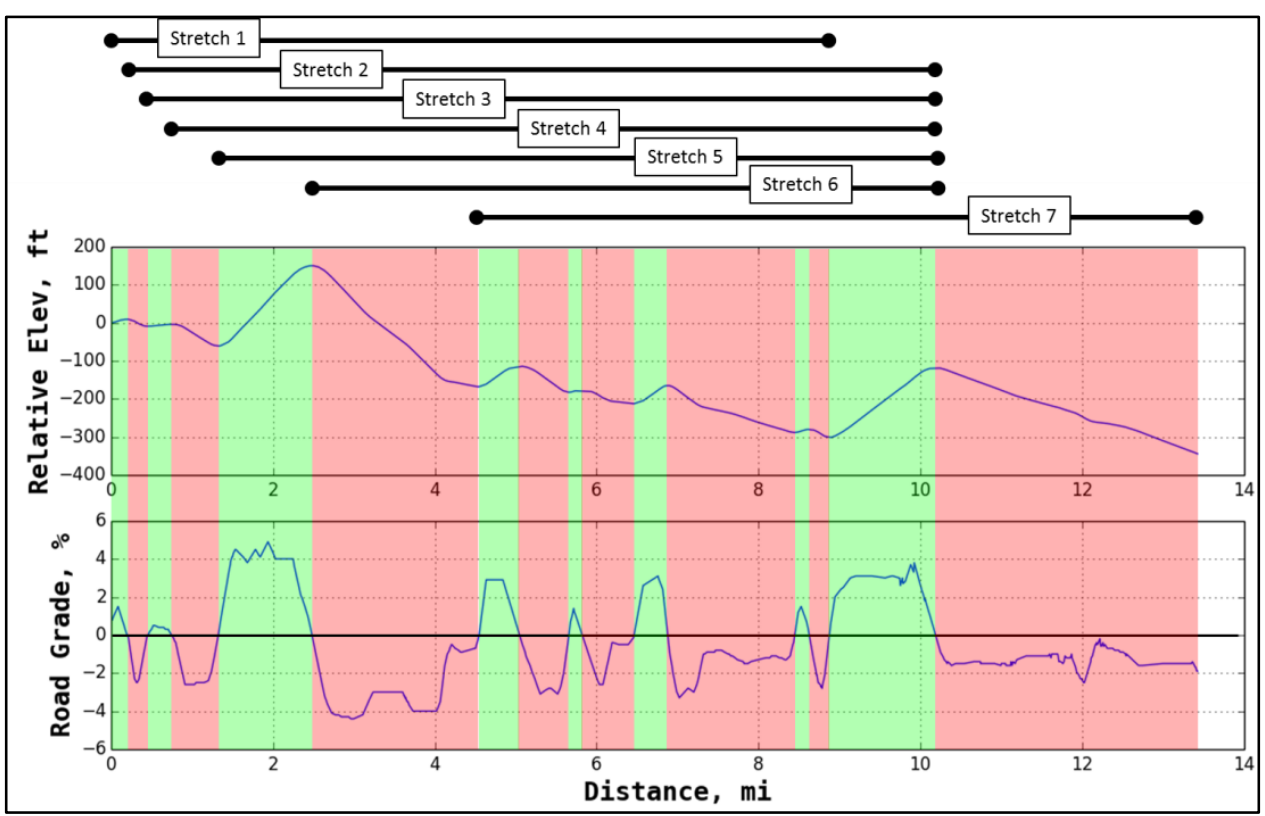

Figure 42. Example 13-mile stretch of roadway segmented into 16 half hills and grouped into seven continuous stretches of 10 miles or less

For this exercise, input segment distance was swept from 5 to 20 miles in 1-mile increments, resulting in inspection of approximately 3-million unique segments, and segments where absolute road grade and half hill distance KS statistics were below 0.10 and 0.15 , respectively, were recorded. In order to satisfy the requirement that example grade profiles feature net zero elevation, local routes in this section would necessarily be mirrored to reflect round-trip travel over a stretch of road (effectively doubling the distance of the example profile).

Additionally, a handful of criteria were imposed during the identification of local routes to facilitate potential on-road testing, including:

- Routes must feature identical grade profile in both directions of travel (to ensure net zero elevation change during on-road testing in support of the assumption that one-way profiles can be mirrored to create round-trip profiles)

- Prefer routes in areas designated by the U.S. Census as rural (decrease potential for traffic to interfere with on-road testing)

- Prefer routes south of the $40^{\text {th }}$ parallel north [Kansas/Nebraska border] (decrease potential for winter weather to interfere with on-road testing).

\subsubsection{5-mph Test Group}

For the 55-mph test group, 8,826 local routes were identified with KS statistics less than 0.10 and 0.15 for distributions of absolute road grade and half hill distance, respectively. Local route KS statistics for distributions of absolute road grade and half hill distance are plotted in Figures 43 and 44 versus round-trip profile distance (color bands represent the sweep of input distance limit). As with the generation of synthetic grade profiles, we see a general trend towards lower KS statistics (implying better matches to the national activity-weighted distributions) as route distance is increased. 


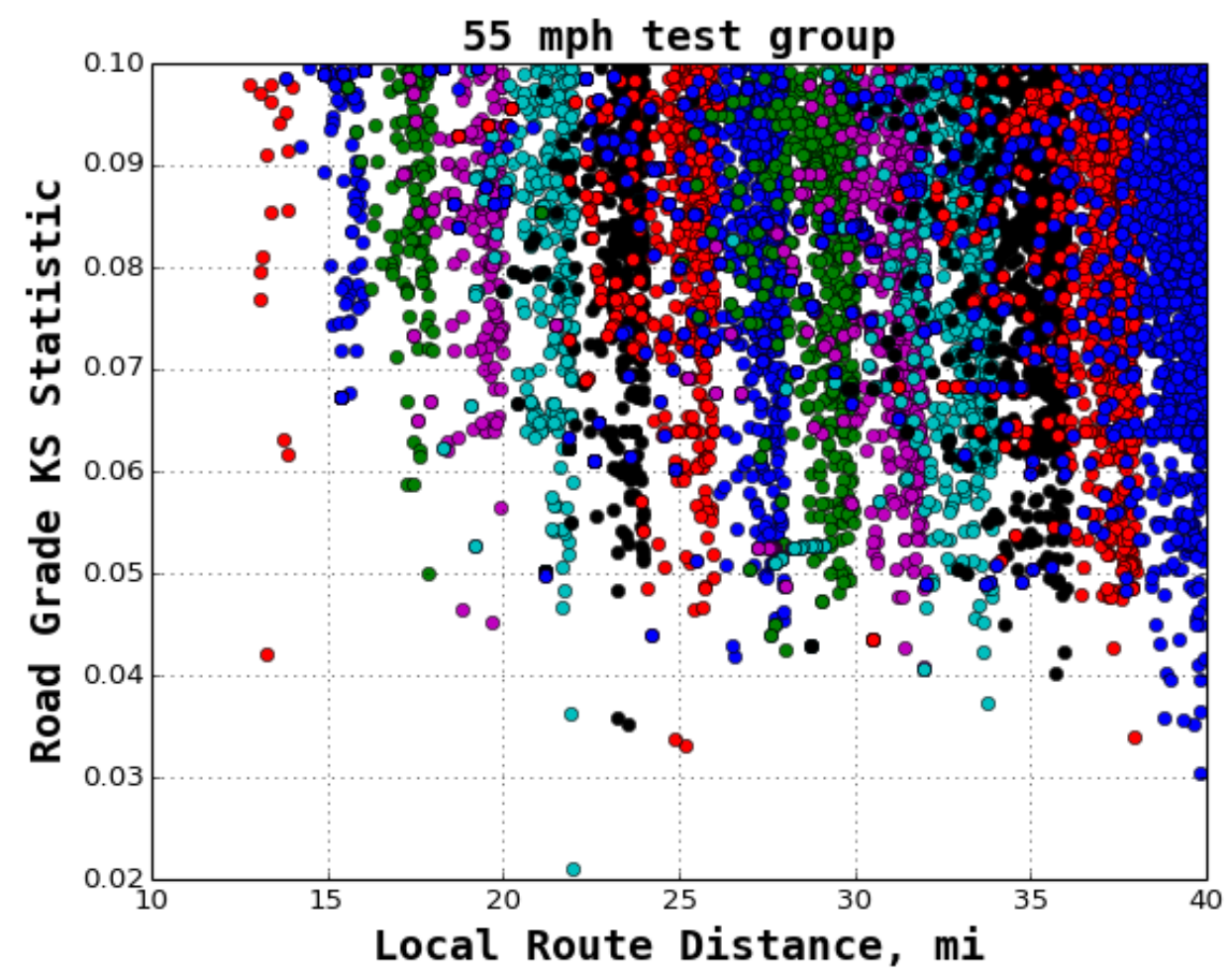

Figure 43. Local route KS statistics for the distribution of absolute road grade versus round-trip profile distance for the 55-mph test group

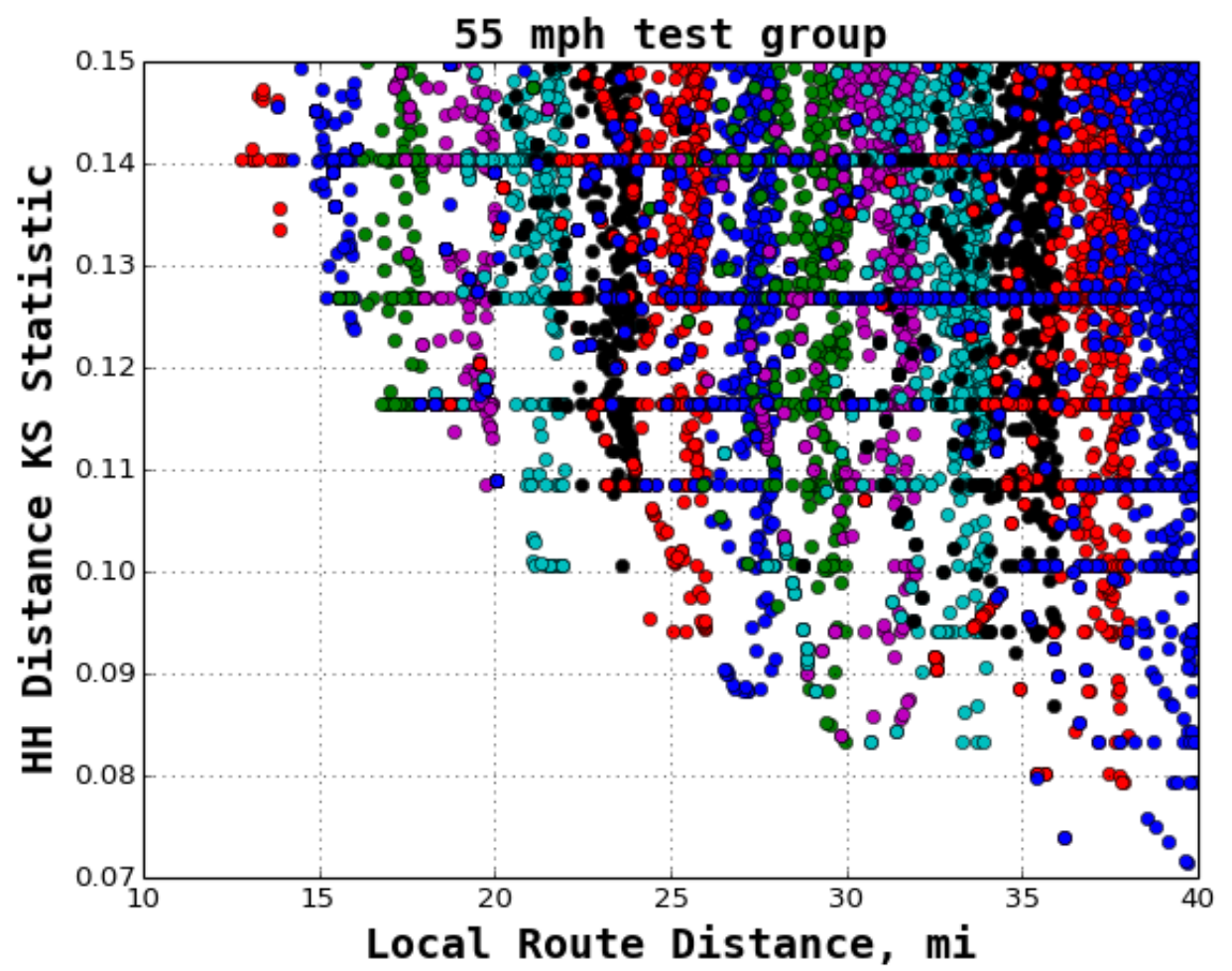

Figure 44. Local route KS statistics for the distribution of half hill distance versus round-trip profile distance for the 55-mph test group 
Only one local route was able to simultaneously achieve KS statistics of less than 0.05 and 0.10 for distributions of absolute road grade and half hill distance, respectively (minimum criteria established for selection of synthetic routes in Section 4.1). Consequently, the performance criteria were adjusted for local routes such that candidate profiles must feature KS statistics of less than 0.05 and 0.15 for distributions of absolute road grade and half hill distance, respectively. This decision reflects the perceived priority for example grade profiles to match national activity-weighted distributions of absolute road grade with a greater level of accuracy relative to distributions of half hill distance.

Three local routes satisfying the new statistical criteria and conditions facilitating on-road testing have been identified:

- I-20 east of Abilene, Texas

- I-70 east of Glenwood Springs, Colorado

- Kentucky State Route (SR) 9001 (Western Kentucky Parkway) south of Louisville, Kentucky

Figures 45 through 47 present the three local routes that are similar to the national activityweighted distributions for the 55-mph test group (segments of interest highlighted in magenta).

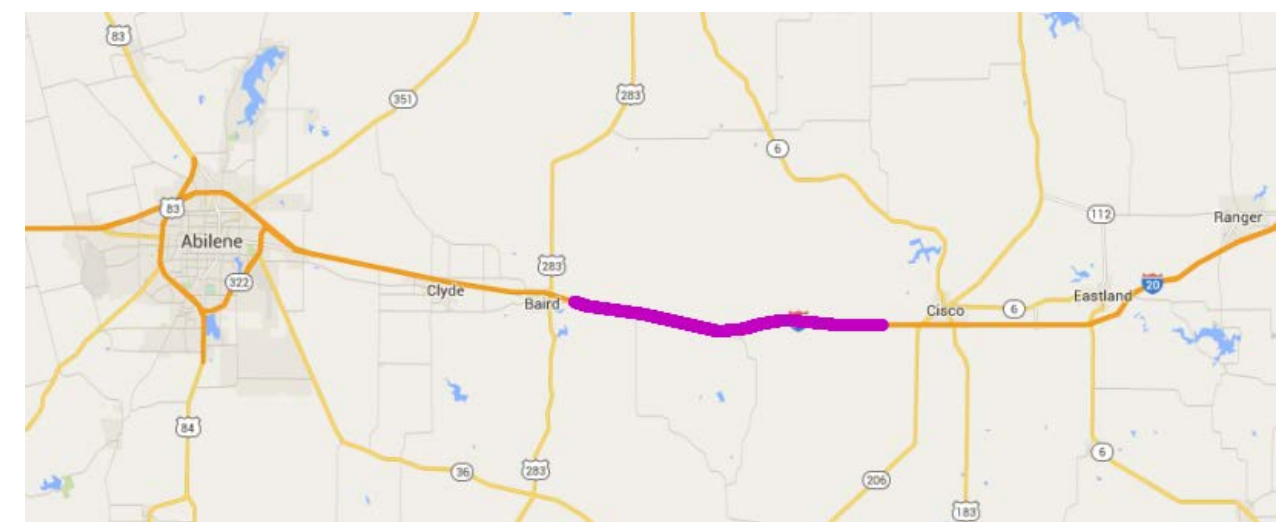

Figure 45. Local route from I-20 east of Abilene, Texas, statistically similar to national activityweighted distributions for the 55-mph test group

(Google Maps credit: @ 2015 Google, Map Data @ 2015 Tele Atlas) 


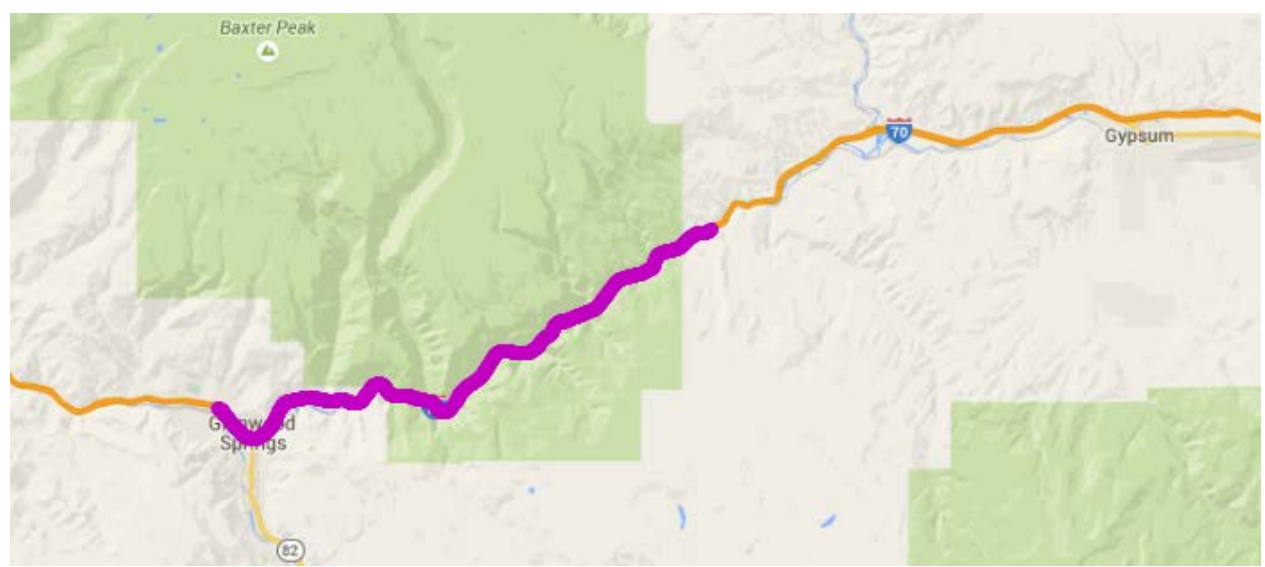

Figure 46. Local route from I-70 east of Glenwood Springs, Colorado, statistically similar to national activity-weighted distributions for the 55-mph test group

(Google Maps credit: @ 2015 Google, Map Data @ 2015 Tele Atlas)

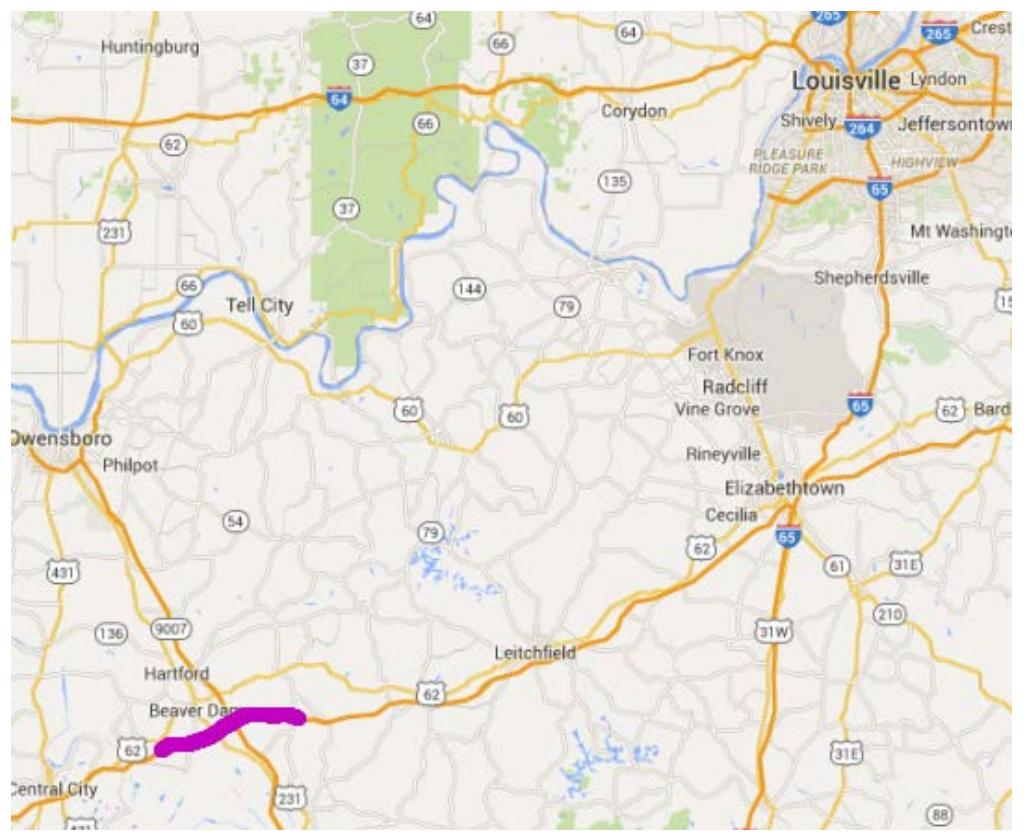

Figure 47. Local route from Kentucky SR-9001 south of Louisville, Kentucky, statistically similar to national activity-weighted distributions for the 55-mph test group

(Google Maps credit: @ 2015 Google, Map Data @ 2015 Tele Atlas)

One-way relative elevation and grade profiles for the three local routes similar to the national activity-weighted distributions for the 55-mph test group are shown in Figures 48 through 50. 

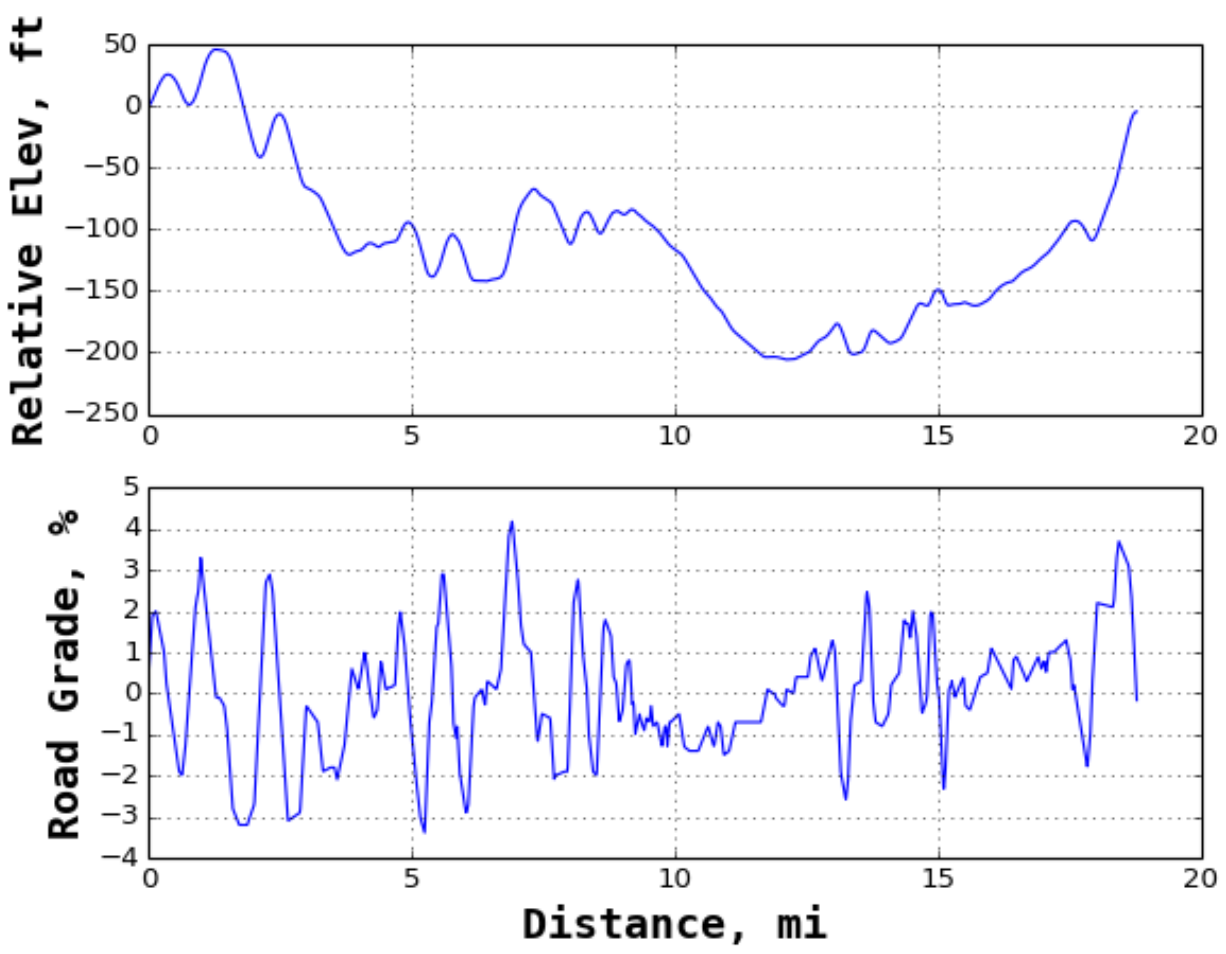

Figure 48. Local route from I-20 east of Abilene, Texas, statistically similar to national activityweighted distributions for the 55-mph test group
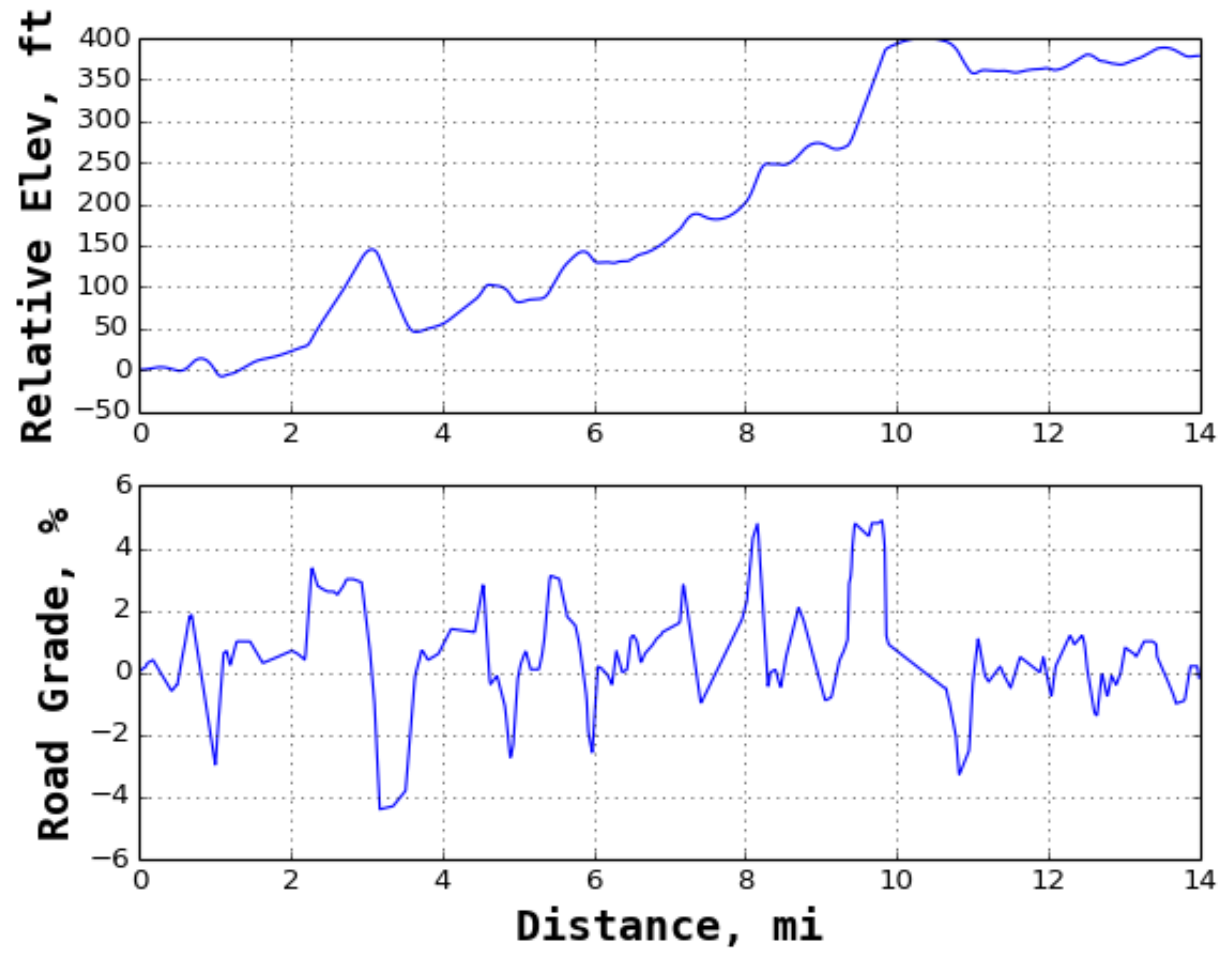

Figure 49. Local route from I-70 east of Glenwood Springs, Colorado, statistically similar to national activity-weighted distributions for the 55-mph test group 

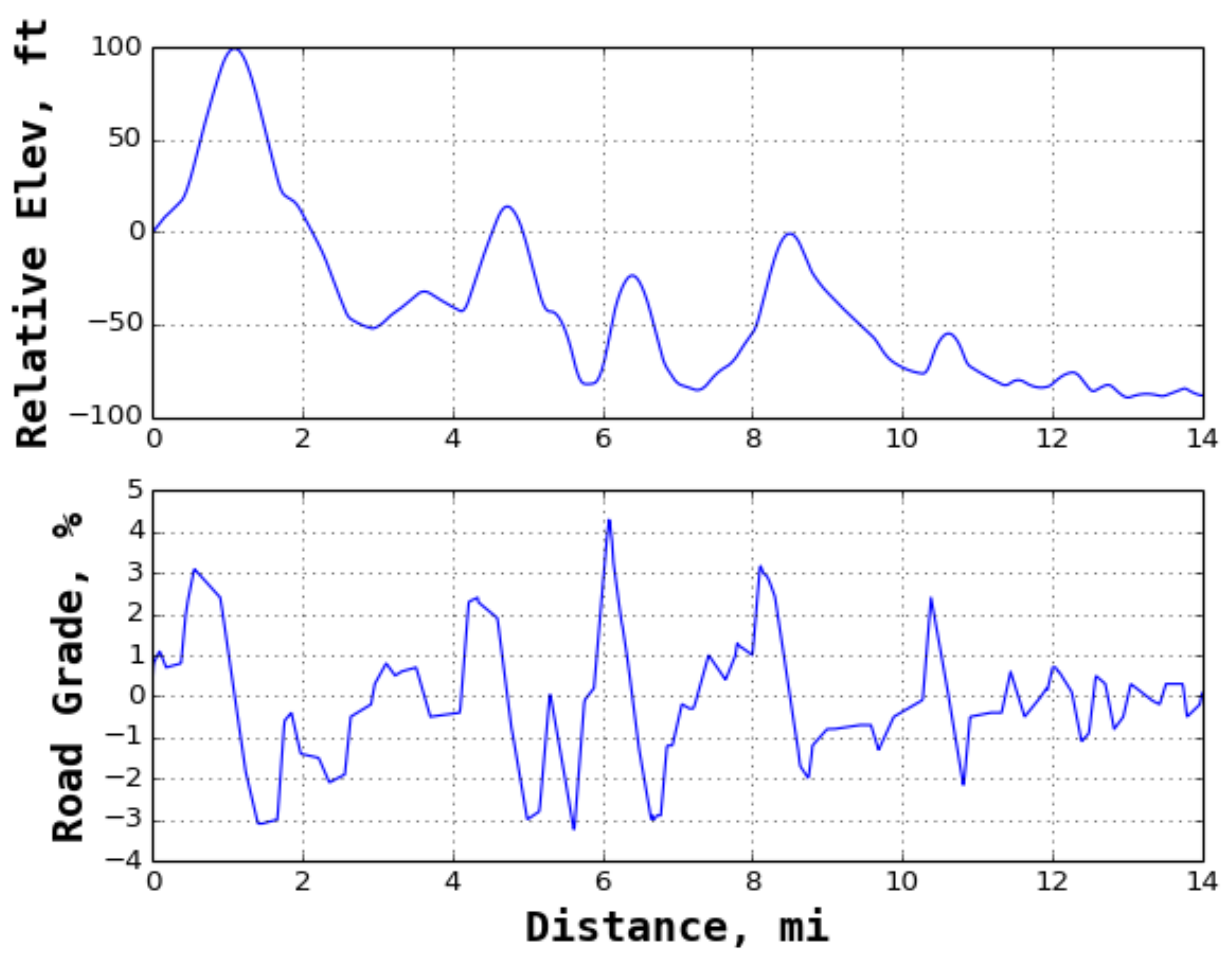

Figure 50. Local route from Kentucky SR-9001 south of Louisville, Kentucky, statistically similar to national activity-weighted distributions for the 55-mph test group

Graphical representations of the cumulative distributions of absolute road grade and half hill distance from the three local routes similar to the national activity-weighted distributions for the 55-mph test group can be found in Figures 51 through 53.
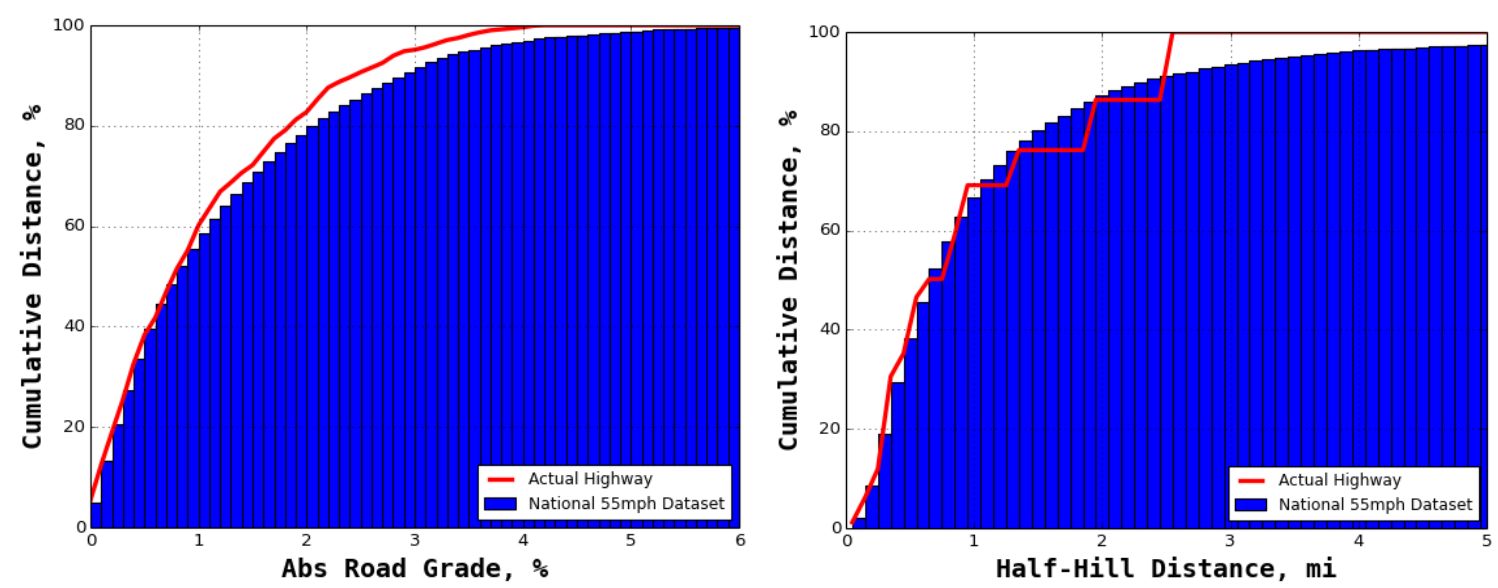

Figure 51. Comparison of distance-based cumulative distributions between national activityweighted dataset and local profile from I-20 east of Abilene, Texas, in terms of absolute road grade (left) and half hill distance (right) 

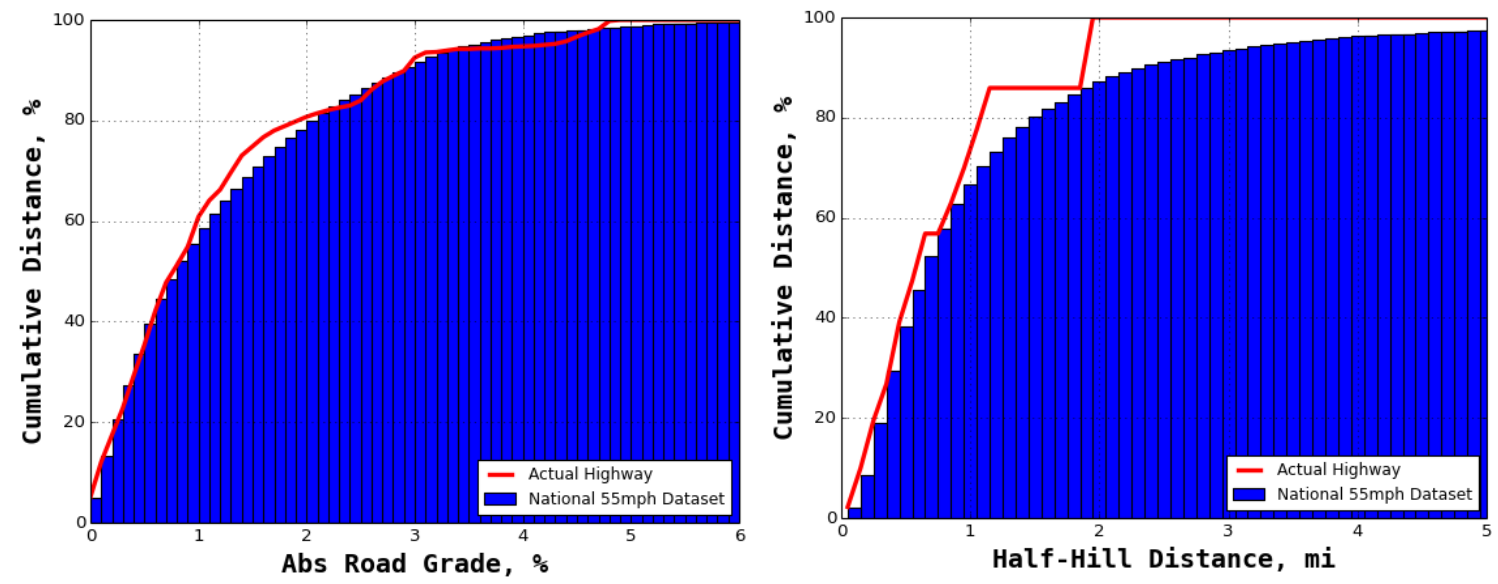

Figure 52. Comparison of distance-based cumulative distributions between national activityweighted dataset and local profile from I-70 east of Glenwood Springs, Colorado, in terms of absolute road grade (left) and half hill distance (right)
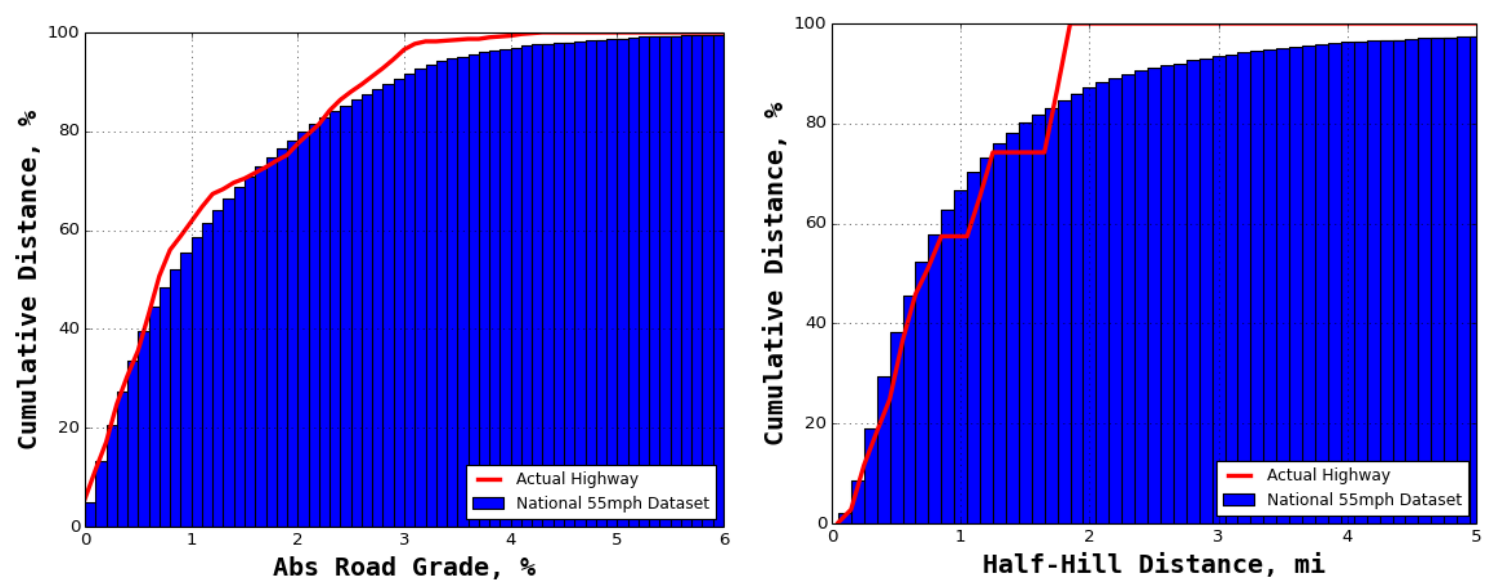

Figure 53. Comparison of distance-based cumulative distributions between national activityweighted dataset and local profile from Kentucky SR-9001 south of Louisville, Kentucky, in terms of absolute road grade (left) and half hill distance (right) 
Summary statistics for the three local routes identified for the 55-mph test group are provided in Table 2, including: one-way and round-trip distances, segment start and end coordinates, and KS hypothesis test results. All three local routes passed KS hypothesis testing at a 95\% confidence level for similarity to the national activity-weighted distributions of absolute road grade and half hill distance (i.e., failed to reject the null hypothesis that the local and national distributions are equivalent).

Table 2. Summary of three local routes statistically similar to national activity-weighted distributions for the 55-mph test group

\begin{tabular}{lccc}
\cline { 2 - 4 } & $\begin{array}{l}\text { I-20 east of } \\
\text { Abilene, TX }\end{array}$ & $\begin{array}{c}\text { I-70 east of } \\
\text { Glenwood } \\
\text { Springs, Co }\end{array}$ & $\begin{array}{c}\text { Kentucky SR- } \\
\text { 9001 south of } \\
\text { Louisville, KY }\end{array}$ \\
\hline One-way distance, mi & 18.8 & 14.0 & 14.0 \\
Round-trip distance, mi & 37.5 & 28.0 & 28.0 \\
\hline Segment start latitude & 32.3756 & 39.5586 & 37.3885 \\
Segment start longitude & -99.0450 & -107.3395 & -86.6981 \\
Segment end latitude & 32.3957 & 39.6166 & 37.3444 \\
Segment end longitude & -99.3620 & -107.1319 & -86.9353 \\
\hline KS statistic (grade) & 0.048 & 0.046 & 0.049 \\
P-value (grade) & 0.027 & 0.052 & 0.054 \\
Hypo Test Result (grade) & Fail to reject & Fail to reject & Fail to reject \\
\hline KS statistic (HH dist) & 0.098 & 0.128 & 0.140 \\
P-value (HH dist) & 0.464 & 0.328 & 0.390 \\
Hypo test result (HH dist) & Fail to reject & Fail to reject & Fail to reject \\
\hline
\end{tabular}




\subsubsection{5-mph Test Group}

For the 65 -mph test group, 7,424 local routes were identified with KS statistics less than 0.10 and 0.15 for distributions of absolute road grade and half hill distance, respectively. Local route KS statistics for distributions of absolute road grade and half hill distance are plotted in Figures 54 and 55 versus round-trip profile distance (color bands represent the sweep of input distance limit). As with the generation of synthetic grade profiles, we see a general trend towards lower KS statistics (implying better matches to the national activity-weighted distributions) as route distance is increased.

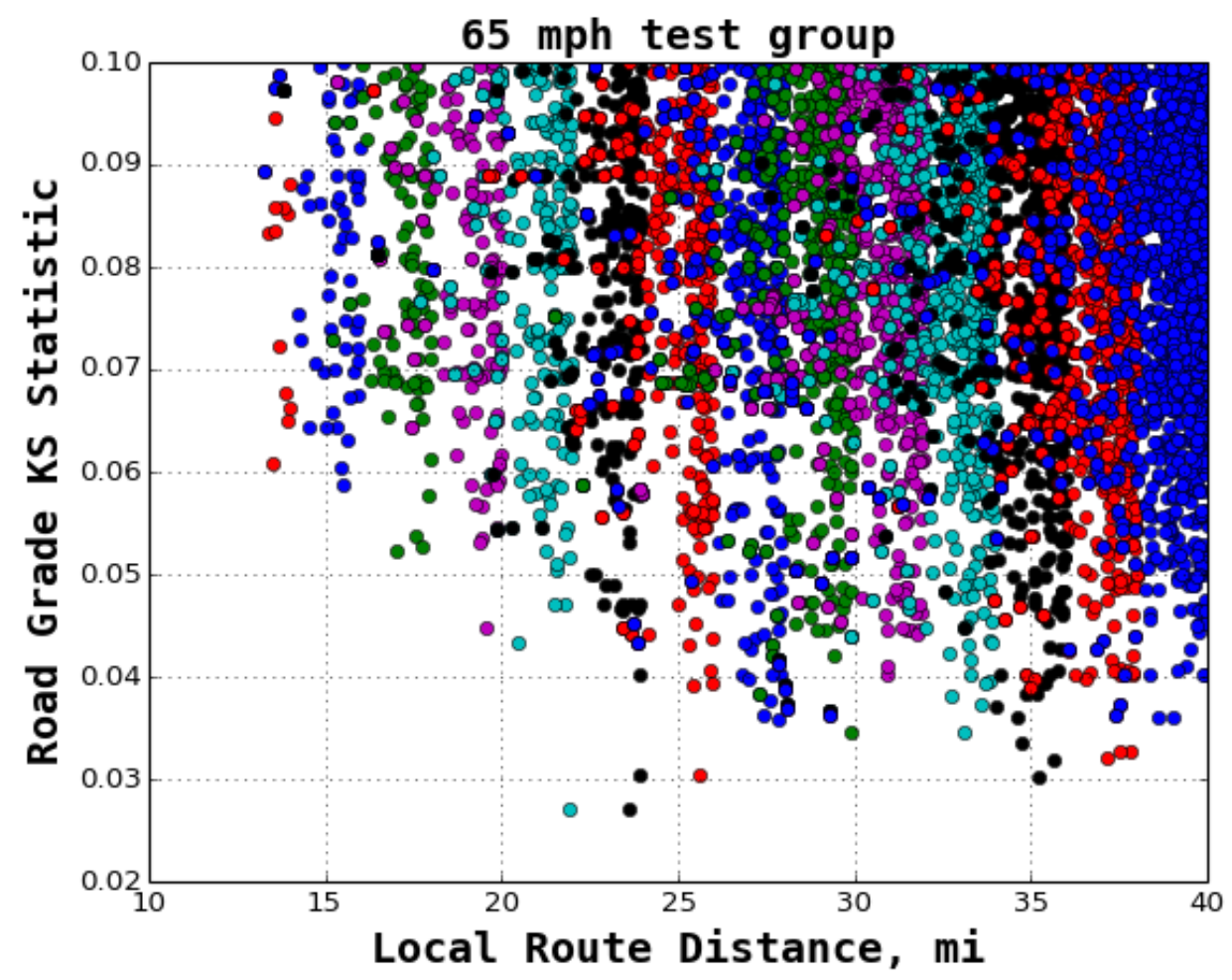

Figure 54. Local route KS statistics for the distribution of absolute road grade versus round-trip profile distance for the 65-mph test group 


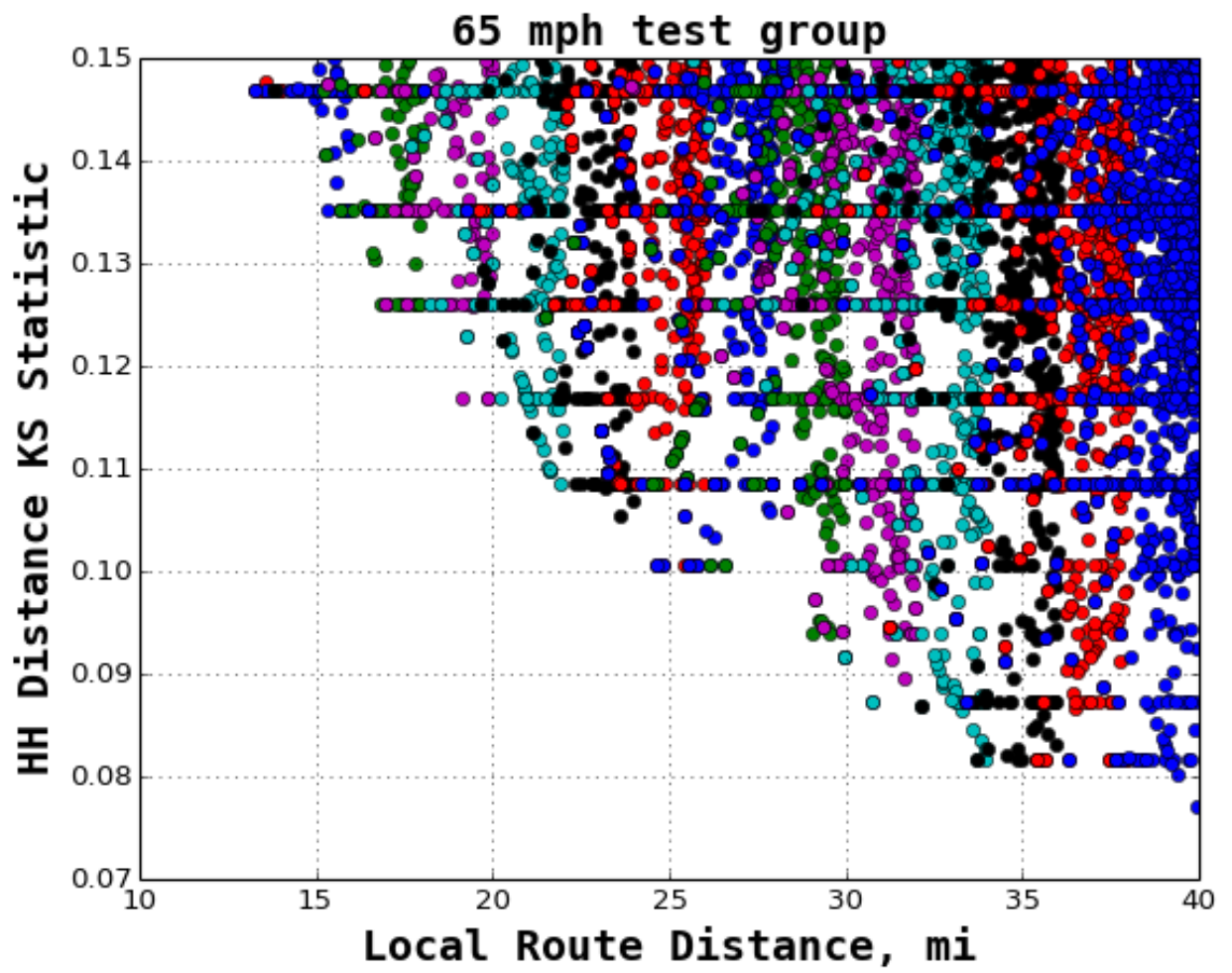

Figure 55. Local route KS statistics for the distribution of half hill distance versus round-trip profile distance for the 65-mph test group

Only one local route was able to simultaneously achieve KS statistics of less than 0.05 and 0.10 for distributions of absolute road grade and half hill distance, respectively (minimum criteria established for selection of synthetic routes in Section 4.1). Consequently, the performance criteria were adjusted for local routes such that candidate profiles must feature KS statistics of less than 0.05 and 0.15 for distributions of absolute road grade and half hill distance, respectively. This decision reflects the perceived priority for example grade profiles to match national activity-weighted distributions of absolute road grade with a greater level of accuracy relative to distributions of half hill distance.

Two local routes satisfying the statistical criteria and conditions facilitating on-road testing have been identified:

- I-10 west of San Antonio, Texas

- I-40 west of Gallup, New Mexico

Maps of the two local routes similar to the national activity-weighted distributions for the 65 mph test group are shown in Figures 56 and 57 (segment of interest highlighted in magenta). 


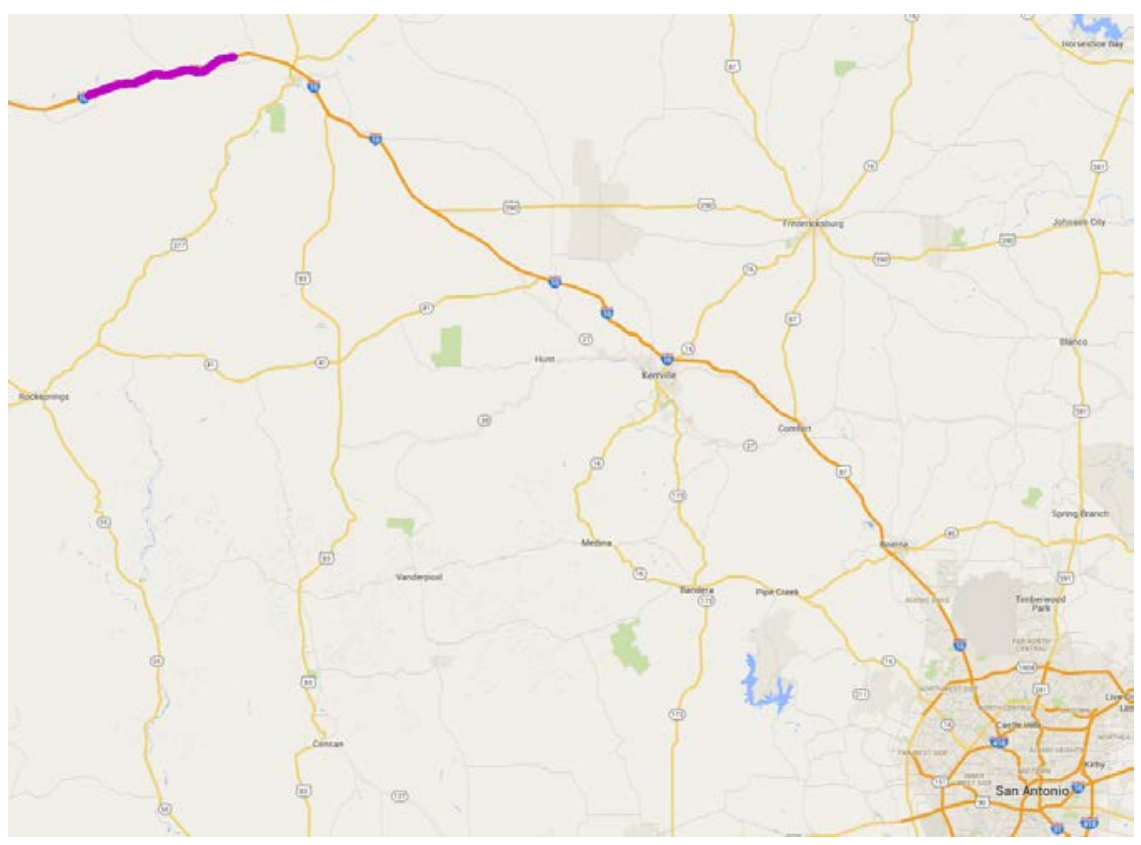

Figure 56. Local route from I-10 west of San Antonio, Texas, statistically similar to national activity-weighted distributions for the 65-mph test group

(Google Maps credit: @ 2015 Google, Map Data @ 2015 Tele Atlas)

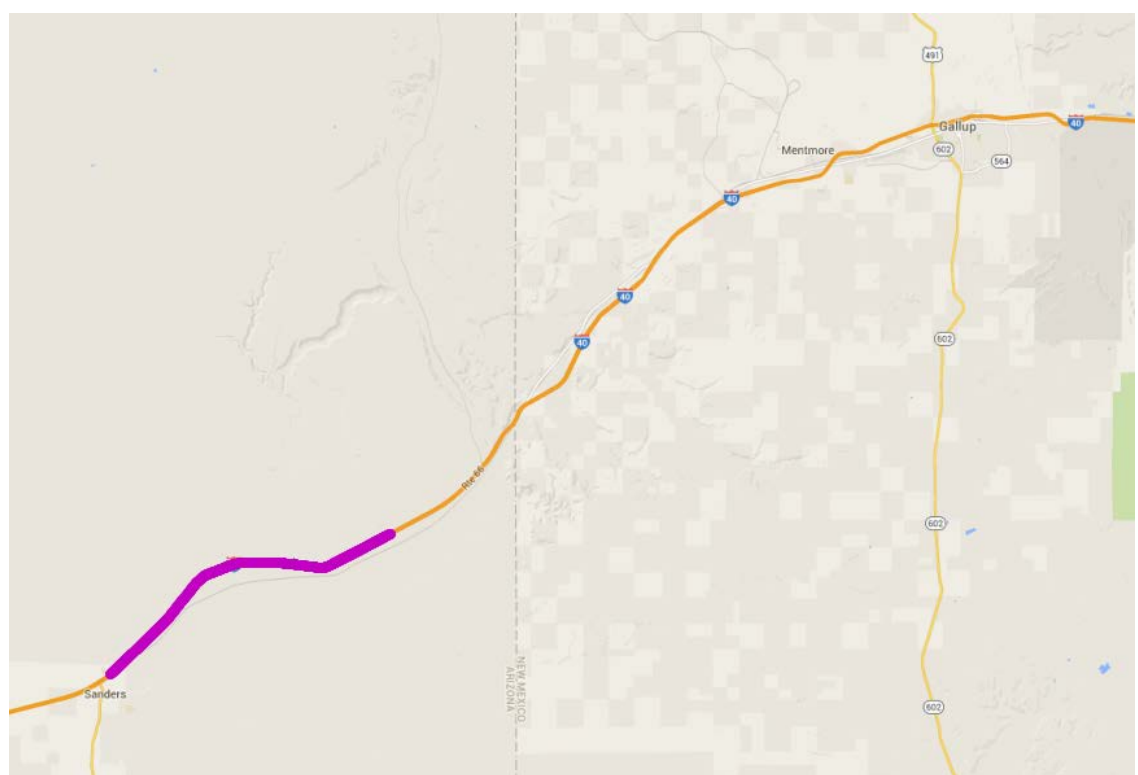

Figure 57. Local route from I-40 west of Gallup, New Mexico, statistically similar to national activity-weighted distributions for the 65-mph test group

(Google Maps credit: @ 2015 Google, Map Data (c 2015 Tele Atlas)

One-way relative elevation and grade profiles for the two local routes similar to the national activity-weighted distributions for the 65-mph test group are shown in Figures 58 and 59. 

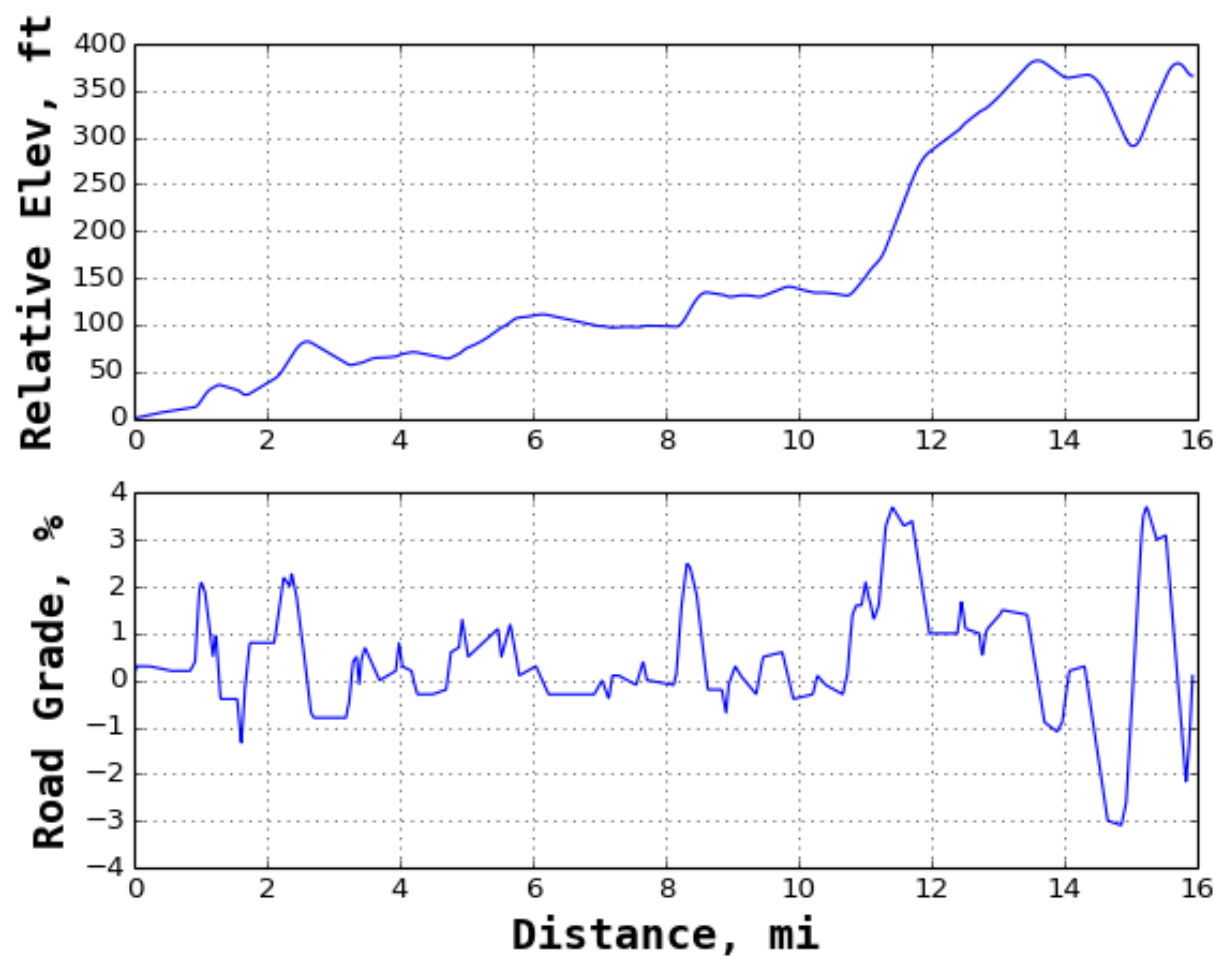

Figure 58. Local route from I-10 west of San Antonio, Texas, statistically similar to national activity-weighted distributions for the 65-mph test group
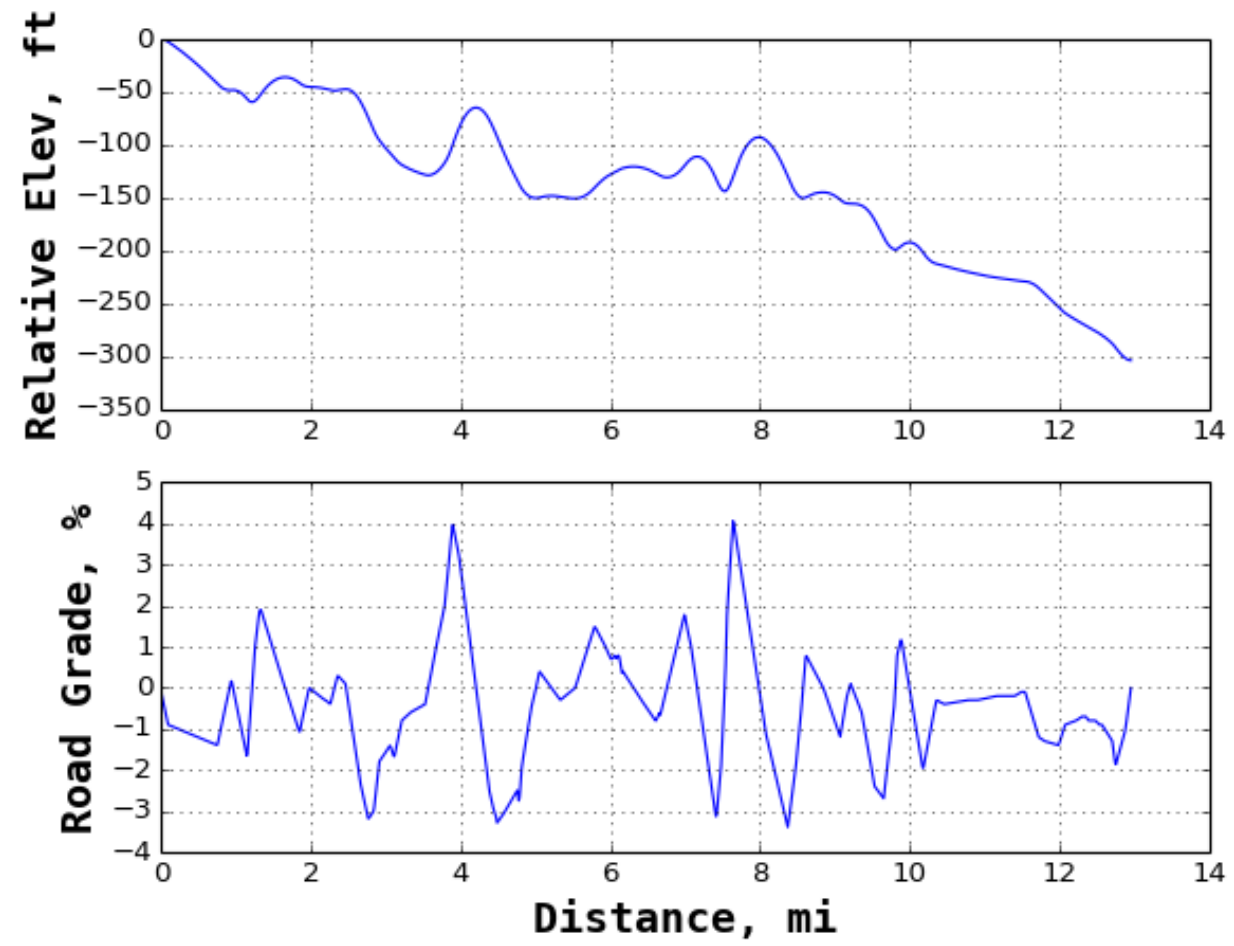

Figure 59. Local route from I-40 west of Gallup, New Mexico, statistically similar to national activity-weighted distributions for the 65-mph test group 
Graphical representations of the cumulative distributions of absolute road grade and half hill distance from the two local routes similar to the national activity-weighted distributions for the 65-mph test group are shown in Figures 60 and 61.
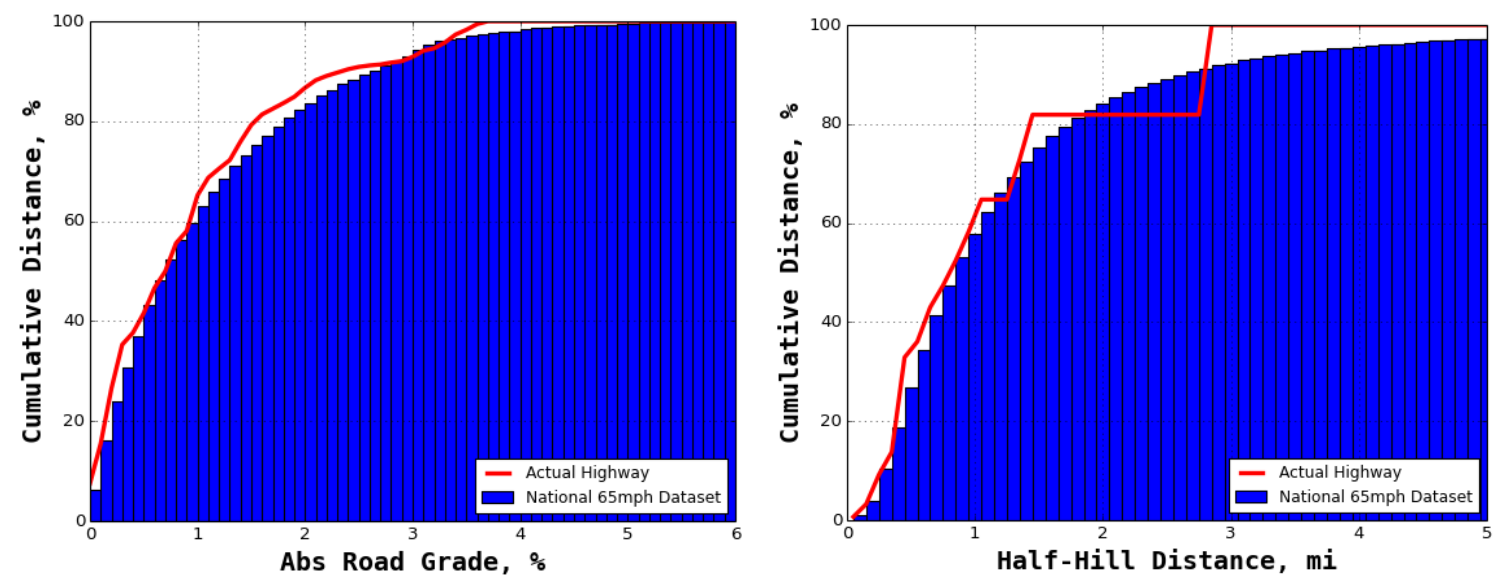

Figure 60. Comparison of distance-based cumulative distributions between national activityweighted dataset and local profile from I-10 west of San Antonio, Texas, in terms of absolute road grade (left) and half hill distance (right)
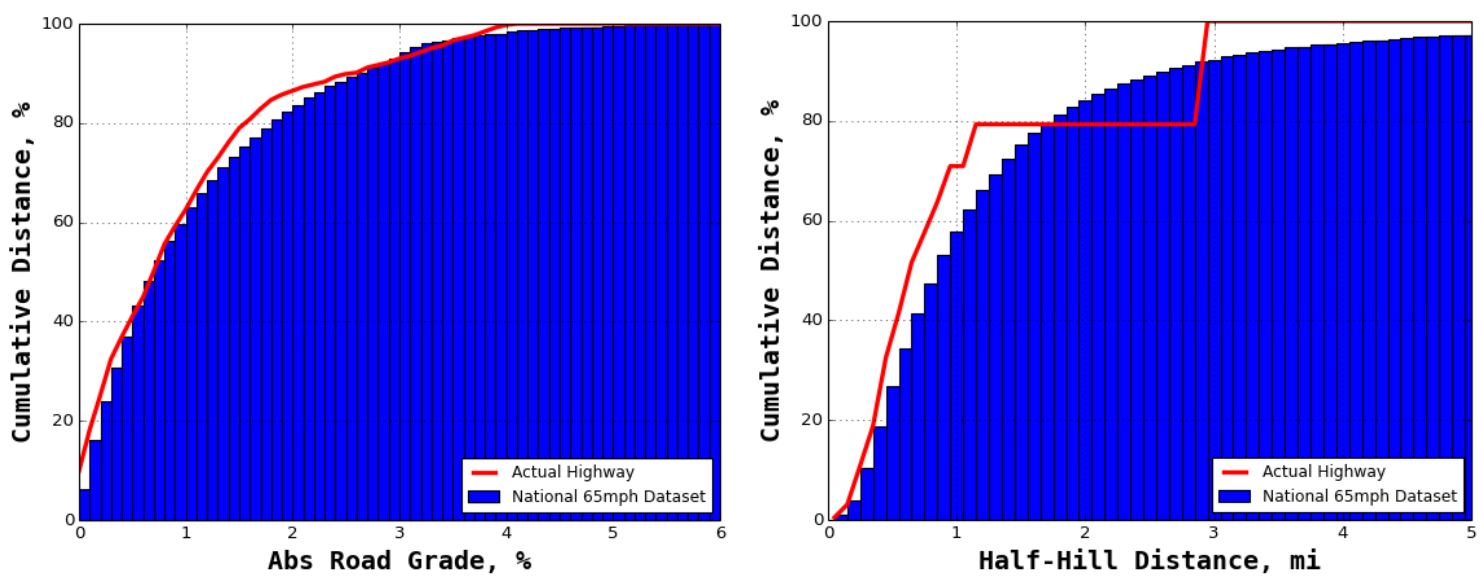

Figure 61. Comparison of distance-based cumulative distributions between national activityweighted dataset and local profile from I-40 west of Gallup, New Mexico, in terms of absolute road grade (left) and half hill distance (right) 
Summary statistics for the two local routes identified for the 65 -mph test group are provided in Table 3 including: one-way and round-trip distances, segment start and end coordinates, and KS hypothesis test results. Both local routes passed KS hypothesis testing at a $95 \%$ confidence level for similarity to the national activity-weighted distributions of absolute road grade and half hill distance (i.e., failed to reject the null hypothesis that the local and national distributions are equivalent).

Table 3. Summary of two local routes statistically similar to national activity-weighted distributions for the 65-mph test group

\begin{tabular}{|c|c|c|}
\hline & $\begin{array}{l}\text { I-10 west of San } \\
\text { Antonio, Texas }\end{array}$ & $\begin{array}{l}\text { I-40 west of Gallup, } \\
\text { New Mexico }\end{array}$ \\
\hline One-way Distance, mi & 15.9 & 13.0 \\
\hline Round-trip Distance, mi & 31.9 & 25.9 \\
\hline$\overline{\text { Segment Start Latitude }}$ & 30.5226 & 35.2994 \\
\hline Segment Start Longitude & -99.8762 & -109.1330 \\
\hline Segment End Latitude & 30.4654 & 35.2204 \\
\hline Segment End Longitude & -100.1276 & -109.3259 \\
\hline KS Statistic (grade) & 0.046 & 0.041 \\
\hline P-Value (grade) & 0.031 & 0.274 \\
\hline Hypo Test Result (grade) & Fail to reject & Fail to reject \\
\hline$\overline{\text { KS Statistic (hh dist) }}$ & 0.094 & 0.147 \\
\hline P-Value (hh dist) & 0.599 & 0.370 \\
\hline Hypo Test Result (hh dist) & Fail to reject & Fail to reject \\
\hline
\end{tabular}




\section{Known Limitations and Potential for Future Work}

Development of sample grade profiles in this analysis has been intended to be directly coupled to constant-speed drive cycles at 55- and 65-mph for EPA certification testing and simulation activities. This type of duty cycle (constant speeds on positive and negative grades) is known to misrepresent real-world operation of MD/HD trucks.

Whether due to driving style or vehicle power limitations, on-road data suggest there is a correlation between road grade and transient highway speeds for MD/HD trucks. This observation is consistent with the SwRI on-road testing of a class 8 combination truck near San Antonio, Texas (described in Section 2.1.1). Figure 62 provides a 10-minute snapshot of this testing at highway speeds and over a number of steep hills. The correlation between vehicle speed and road grade is highlighted for the six instances where the measured road grade exceeded $+4 \%$. Vehicle speed consistently drops on these sections of climb (from approximately 75 to $55 \mathrm{mph}$ in the last instance), and on three occasions this drop in vehicle speed coincides with a transmission down-shifting event.

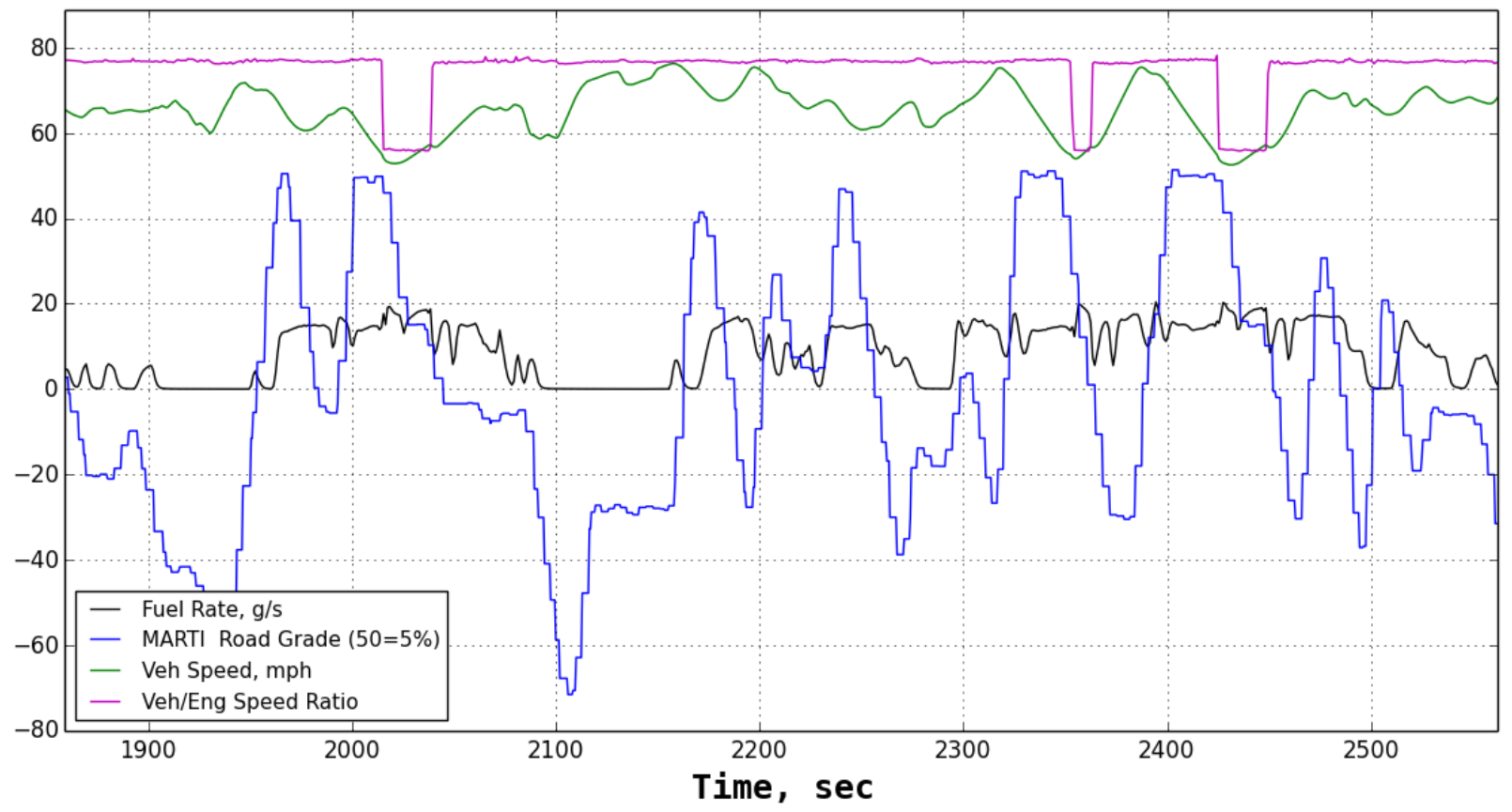

Figure 62. Vehicle fueling rate, road grade, vehicle speed, and vehicle to engine speed ratio for a 10-minute section of SwRI on-road testing of a class 8 combination truck

Given the apparent mismatch between dynamometer testing at constant highway speeds over varying road grade and observed real-world operation, NREL and the EPA have identified the need to refine existing vehicle test speeds for highway cycles at grade. Development of a fully integrated speed and grade duty cycle for highway testing and simulation is the focus of an ongoing project between NREL and EPA. 


\section{References}

[1] U.S. Environmental Protection Agency. (2011). "EPA and NHTSA Adopt First-Ever Program to Reduce Greenhouse Gas Emissions and Improve Fuel Efficiency of Medium- and Heavy-Duty Vehicles." EPA-420-F-11-031. Office of Transportation and Air Quality. Accessed February 16, 2015. http://www.epa.gov/otaq/climate/documents/420f11031.pdf

[2] U.S. Environmental Protection Agency. (2011). "Final Rulemaking to Establish Greenhouse Gas Emission Standards and Fuel Efficiency Standards to Medium- and Heavy-Duty Engines and Vehicles: Regulatory Impact Analysis.” EPA-420-R-11901. Office of Transportation and Air Quality and National Highway Traffic Safety Administration. Accessed February 16, 2015. http://www.epa.gov/otaq/climate/documents/420r11901.pdf

[3] Federal Register. (2011). "Greenhouse Gas Emissions Standards and Fuel Efficiency Standards for Medium- and Heavy-Duty Engines and Vehicles: Final Rule." Environmental Protection Agency and Department of Transportation. (76:179); Accessed February 16, 2015. http://www.gpo.gov/fdsys/pkg/FR-2011-0915/pdf/2011-20740.pdf

[4] U.S. Environmental Protection Agency. (February 10, 2015). "Transportation and Climate: Regulations \& Standards: Heavy-Duty.” Office of Transportation and Air Quality. Accessed February 16, 2015. http://www.epa.gov/otaq/climate/regs-heavyduty.htm

[5] The White House, Office of the Press Secretary. (February 18, 2014). "Remarks by the President on Fuel Efficiency Standards of Medium and Heavy-Duty Vehicles." Accessed February 16, 2015. http://www.whitehouse.gov/the-pressoffice/2014/02/18/remarks-president-fuel-efficiency-standards-medium-and-heavyduty-vehicl

[6] The White House, Office of the Press Secretary. (February 18, 2014). "FACT SHEET: Opportunity for All: Improving the Fuel Efficiency of American Trucks Bolstering Energy Security, Cutting Carbon Pollution, Saving Money and Supporting Manufacturing Innovation.” Accessed February 16, 2015. http://www.whitehouse.gov/the-press-office/2014/02/18/fact-sheet-opportunity-allimproving-fuel-efficiency-american-trucks-bol

[7] “Transportation Secure Data Center." (2014). National Renewable Energy Laboratory. Accessed October 31, 2014: www.nrel.gov/tsdc

[8] Walkowicz, K.; Kelly, K.; Duran, A.; Burton, E. (2014). Fleet DNA Project Data. National Renewable Energy Laboratory. http://www.nrel.gov/fleetdna 
[9] National Renewable Energy Laboratory. (2013). "DRIVE Analysis Tool Generates Custom Vehicle Drive Cycles Based on Real-World Data." NREL/FS-5400-54507. Golden, CO: National Renewable Energy Laboratory. Accessed February 16, 2015. http://www.nrel.gov/docs/fy13osti/54507.pdf

[10] Boriboonsomsin, K. and Barth, M. "Impacts of Road Grade on Fuel Consumption and Carbon Dioxide Emissions Evidenced by Use of Advanced Navigation Systems" Transportation Research Record: Journal of the Transportation Research Board, No. 2139, Transportation Research Board of the National Academies, Washington, D.C., 2009, pp. 21-30. DOI: 10.3141/2139-03 http://trb.metapress.com/content/g77x60gv21123376/?genre=article\&id=doi\%3a10.3 $\underline{141 \% 2 \mathrm{f} 2139-03}$

[11] Boroujeni, B.Y. and Frey, H.C. "Road grade quantification based on global positioning system data obtained from real-world vehicle fuel use and emissions measurements" Atmospheric Environment 85 (2014) 179-186, http://www.sciencedirect.com/science/article/pii/S1352231013009709\#

[12] Wyatt, D.W., Li, H., and Tate, J.E. "The impact of road grade on carbon dioxide $\left(\mathrm{CO}_{2}\right)$ emission of a passenger vehicle in real-world driving" Transportation Research Part D 32 (2014) 160-170, http://www.sciencedirect.com/science/article/pii/S136192091400100X

[13] Wood, E., Burton, E., Duran, A., and Gonder, J. "Contribution of road grade to the energy use of modern automobiles across large datasets of real-world drive cycles" SAE World Congress, April 2014, 2014-01-1789, http://www.nrel.gov/docs/fy14osti/61108.pdf

[14] Scora, G.A. "Heavy Duty Diesel Particulate Matter and Fuel Consumption Modeling for Transportation Analysis" Doctor of Philosophy Dissertation, University of California Riverside, March 2012, http://escholarship.org/uc/item/4sj7914b

[15] Franzese, O. "Effect of Weight and Roadway Grade on the Fuel Economy of Class-8 Freight Trucks" Oak Ridge National Laboratory Technical Report, ORNL/TM2011/471, October 2011, http://cta.ornl.gov/cta/Publications/Reports/ORNL_TM_2011_471.pdf

[16] Delorme, A., Karbowski, D., Vijayagopal, R., and Sharer, P. "Evaluation of Fuel Consumption Potential of Medium and Heavy Duty Vehicles through Modeling and Simulation" Argonne National Laboratory Technical Report to National Academy of Sciences, October 2009, DEPS-BEES-001, http://www.autonomie.net/docs/6\%20\%20Papers/nas mediumheavyduty 2009.pdf

[17] Ates, M., and Matthews, R., "Coastdown Coefficient Analysis of Heavy-Duty Vehicles and Application to the Examination of the Effects of Grade and Other Parameters on Fuel Consumption," SAE Technical Paper 2012-01-2051, 2012, doi:10.4271/2012-01-2051. http://papers.sae.org/2012-01-2051/ 
[18] Southwest Research Institute. (July 10, 2014). "Mobile Autonomous Robotics Technology Initiative Program.” Accessed February 16, 2015. www.marti.swri.org/

[19] United States Geological Survey. “The National Map.” Accessed April 21, 2014. http://nationalmap.gov/viewer.html

[20] United States Geological Survey. "National Elevation Dataset." Accessed April 21, 2014. http://ned.usgs.gov/

[21] United States Geological Survey. "3D Elevation Program (3DEP).” Accessed March 2, 2015. http://nationalmap.gov/3DEP/

[22] Wood, E.; Burton, E.; Duran, A.; Gonder, J. (2014). Appending High-Resolution Elevation Data to GPS Speed Traces for Vehicle Energy Modeling and Simulation. NREL/TP-5400-61109. Golden, CO: National Renewable Energy Laboratory. http://www.nrel.gov/docs/fy14osti/61109.pdf

[23] “TomTom MultiNet.” (2015). TomTom International. Accessed February 16, 2015. https://www.tomtom.com/en_gb/licensing/products/maps/multinet/

[24] “TomTom Advanced Driving Attributes: Road Grade.” (2015). TomTom International. Accessed February 16, 2015. http://automotive.tomtom.com/en/realtime-maps/adas-advanced-driver-assistance-systems

[25] TomTom International. (2013). MultiNet Advanced Driving Attributes 1.1: Data and Format Specifications. Document Version 1.0.0.

[26] “TomTom Logistics” TomTom International. Accessed March 17, 2015. http://www.tomtom.com/en_gb/licensing/products/enhanced-content/guide/logistics/

[27] Neubauer, J.; Wood, E. "Accounting for the Variation of Driver Aggression in the Simulation of Conventional and Advanced Vehicles." Preprint. NREL/CP-540057503. Presented at SAE World Congress, April 16-18, 2013. Golden, CO: National Renewable Energy Laboratory. 10 pp. http://www.nrel.gov/docs/fy13osti/57503.pdf

[28] Massachusetts Institute of Technology. "Chapter 5: Nonparametric Statistics and Model Selection.” In 6.S085 Statistics for Research Projects: IAP 2015. Accessed February 16, 2015. http://www.mit.edu/ 6.s085/notes/lecture5.pdf

[29] Massachusetts Institute of Technology, Open Courseware, Statistics for Applications. "Section 13: Kolmogorov-Smirnov Test." pp 83-90. Accessed February 16, 2015. http://ocw.mit.edu/courses/mathematics/18-443-statistics-for-applications-fall2006/lecture-notes/lecture14.pdf

[30] Mathworks, MATLAB Documentation. (2015). "kstest2: Two sample KolmogorovSmirnov test." Accessed February 16, 2015. http://www.mathworks.com/help/stats/kstest2.html?refresh=true 


\section{Appendix A: Supporting Documentation}

\section{A.1 Generating Distributions of Road Grade on Basis of Absolute Values}

The impact of road grade on MD/HD truck fuel economy and emissions can generally be categorized into three elevation profiles:

A. Net elevation deltas (change in potential energy between start and end of a given trip)

B. Cumulative elevation deltas (multiple offsetting elevation changes in a trip with no net elevation delta)

C. A combination of types A and B.

These general elevation profiles are shown graphically in Figure A1.
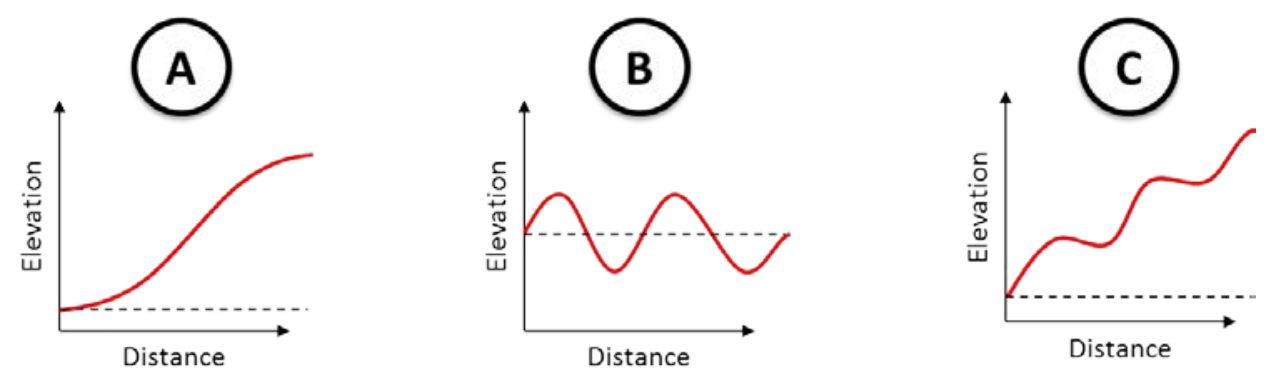

Figure A1. Three generalized categories of elevation profiles

Over the course of a vehicle's lifetime (potentially hundreds of thousands of miles), the impact of net elevation deltas (types A and C) is believed to be essentially negligible. For an extreme example, consider a HD truck with a relatively short service life of one hundred thousand miles that begins its life at sea level (approximate elevation: $0 \mathrm{ft}$.) and ends its life in the Rocky Mountains near the Continental Divide (approximate elevation: 12,000 ft.). This truck would experience a lifetime distance-averaged grade of less than $0.003 \%$.

With this in mind, it becomes clear that a certification test cycle with road grade must contain a net zero elevation delta. Any other cycle would imply perpetual descent or climb (an obviously non-existent attribute of U.S. driving).

Additionally, road grades on such a test cycle can be fully described by their distribution of absolute grades. For example, consider the 2.8-mile stretch of road shown in Figure A2. In addition to exhibiting a net positive change in elevation, inspection of the grade histogram reveals the majority of distance presenting at positive grades. 

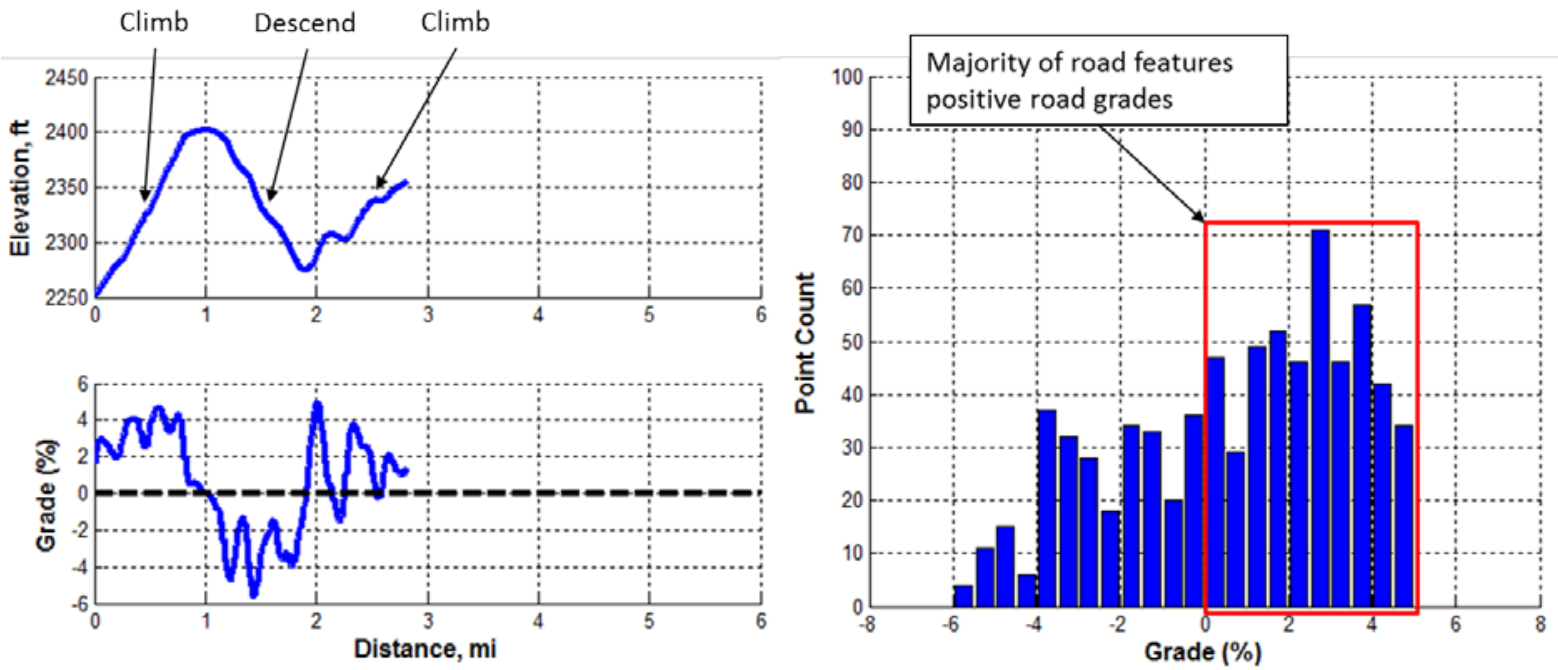

Figure A2. Elevation profile, grade profile, and grade histogram for example 2.8-mile stretch of road

Now consider a round-trip on the same 2.8-mile section of road as shown in Figure A3.

Traveling in both directions over the same stretch of road, the elevation profile is net-zero and a grade histogram is symmetrical about $0 \%$ grade.
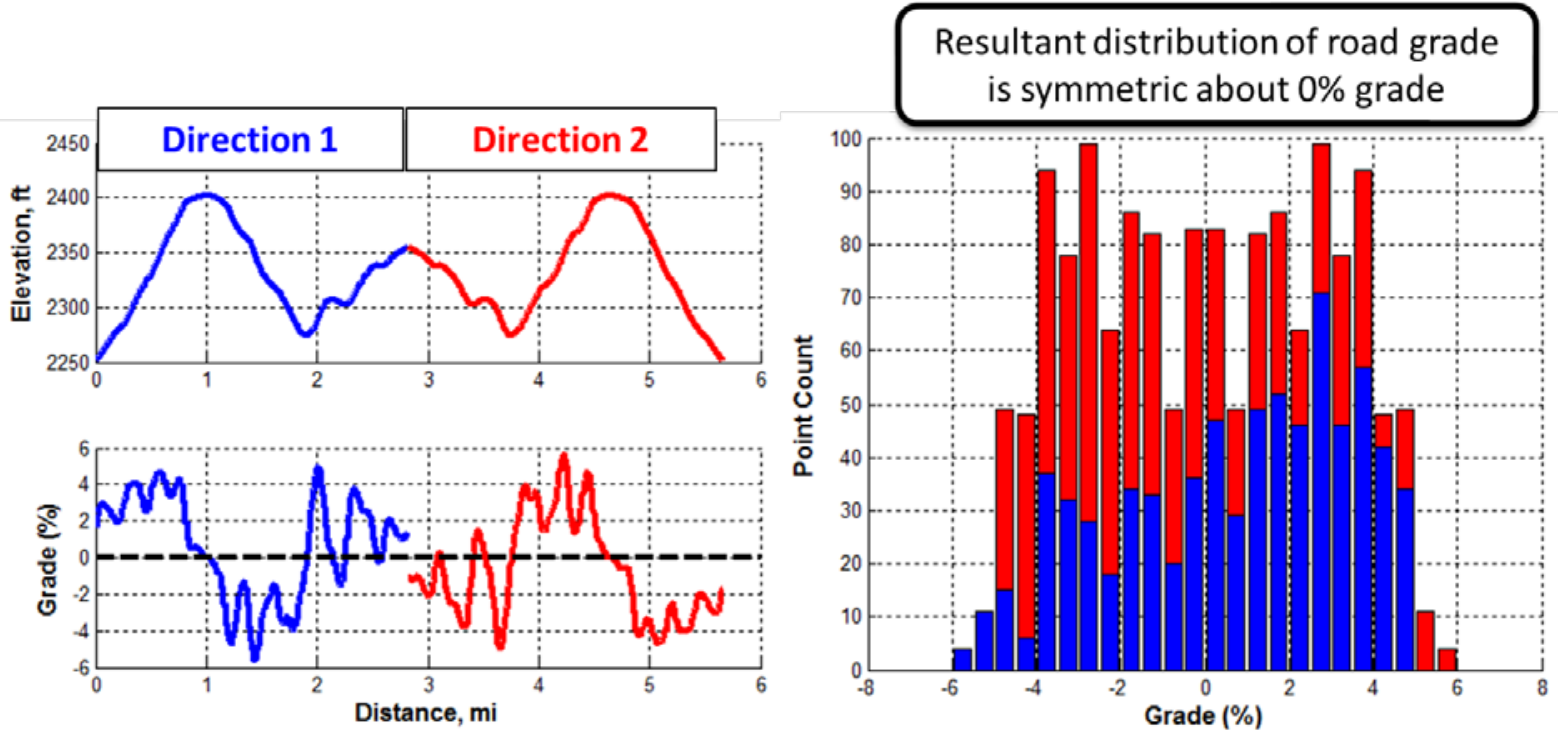

Figure A3. Elevation profile, grade profile, and grade histogram for round trip on example 2.8-mile stretch of road

When considering that round-trip travel on any section of road results in a grade histogram that is symmetric about $0 \%$ grade, it becomes evident that the grade content of any road can be fully described by the histogram of absolute road grade in one direction as shown in Figure A4 for our example roadway. 

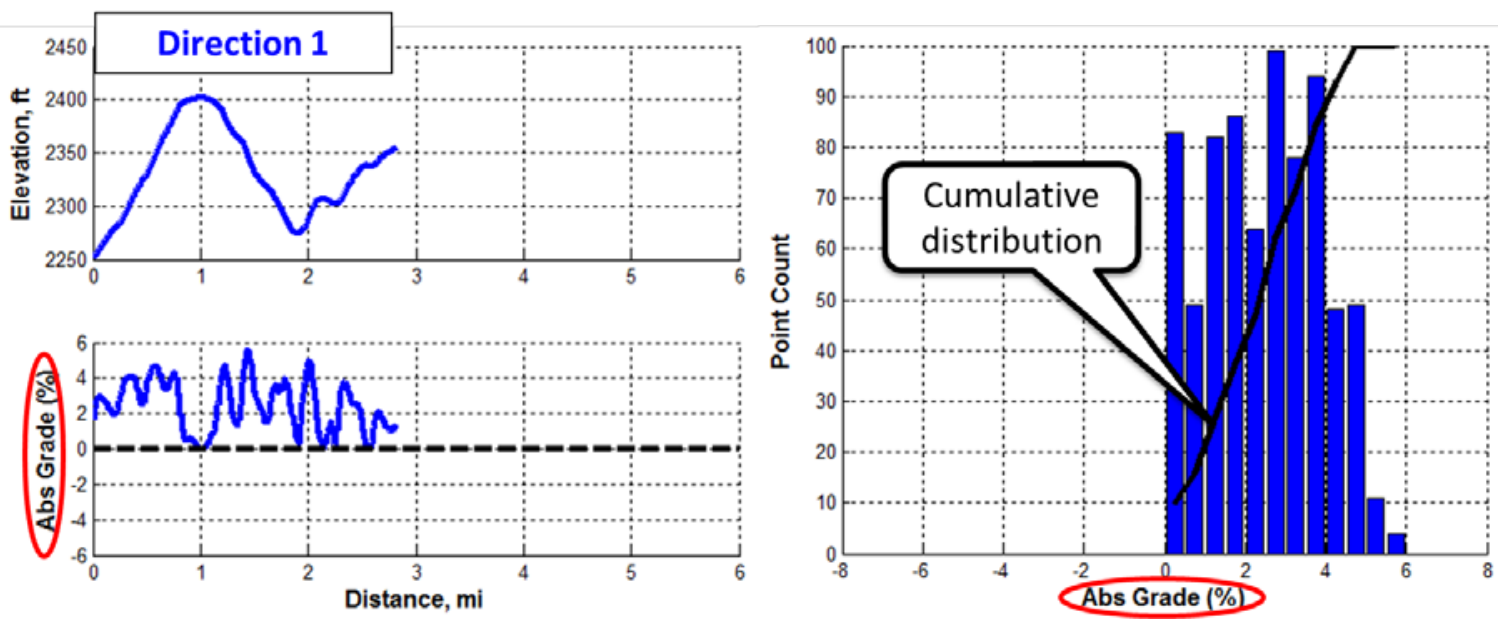

Figure A4. Elevation profile, absolute grade profile, and absolute grade histogram for example 2.8mile stretch of road

\section{A.2 Horizontal versus Projected Roadway Distance}

All distances in this analysis are calculated as the horizontal component of the actual roadway distance. These distance calculations do not take the height or slope of the roadway into account. This methodology is consistent with the road network distances provided in the TomTom MultiNet database and with geographic information system analysis of this type in general.

While road grades on the order of $1 \%-2 \%$ have significant impact on MD/HD vehicle fuel economy and emissions, the impact on driven distance is nearly negligible. For example, calculation of horizontal distance on a $6 \%$ grade underreports driven distance by less than $0.2 \%$. Given the national distribution of road grades presented in this analysis, it is estimated that roadway distance at the national level is underreported by less than $0.02 \%$ as a result of neglecting the vertical component of roadway distance.

\section{A.3 Kolmogorov-Smirnov Hypothesis Testing}

The degree to which sample grade profiles are statistically representative of national data was quantified using Kolmogorov-Smirnov (KS) hypothesis testing. KS testing is a widely used procedure for evaluating the statistical similarity of two cumulative distributions [28-30]. The primary input to KS hypothesis testing is the supremum of the two distributions over the region of interest and is referred to as the KS statistic. The supremum of two distributions is evaluated via Equation A1.

$$
\sup =\max _{x}\left(\operatorname{abs}\left(d_{1}(x)-d_{2}(x)\right)\right)
$$

Where $d_{1}(x)$ is the national cumulative distribution we are targeting to match, $d_{2}(x)$ is the sample cumulative distribution (generated via synthetic or data mining methods), and $\max _{\mathrm{x}}$ is maximum of a one-dimensional array with respect to input variable x (e.g., absolute road grade or half hill distance).

Figure A5 provides an example supremum calculation using the national distance-based, activity-weighted, cumulative distribution of absolute road grade from the 55-mph test group and a synthetically generated sample test profile. The synthetic generation process is described in 
Section 4.1. From this example, we observe that the most significant discrepancy between the national and sample distributions occurs at an absolute road grade of approximately $2.5 \%$.

Approximately $85 \%$ of distances from the national data occur at or below $2.5 \%$ absolute grade, while $95 \%$ of the sample profile distance occurs at or below $2.5 \%$ grade. Calculating the supremum of these two distributions results in a KS statistic of approximately 0.10 (or $10 \%$ ).

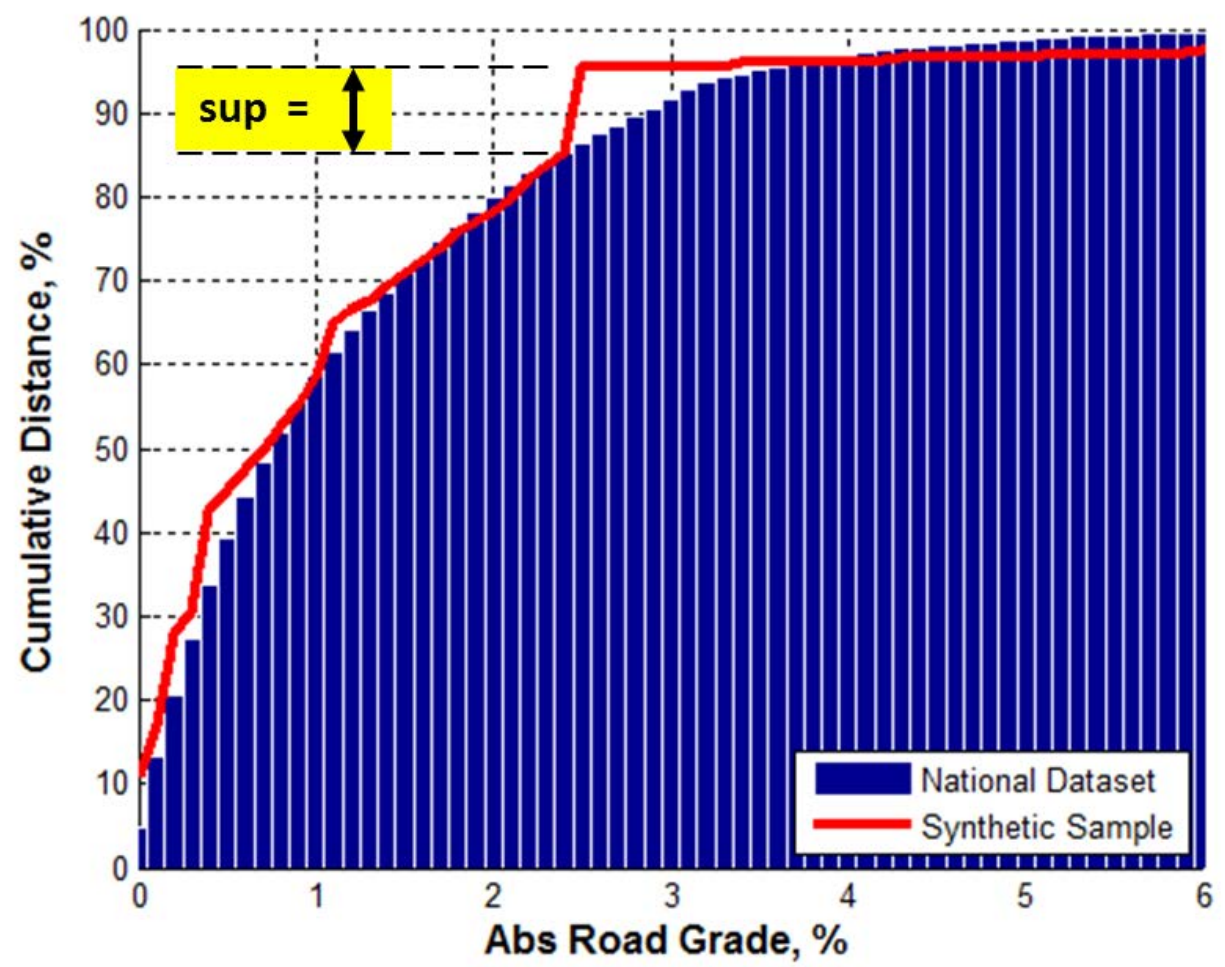

Figure A5. Supremum measurement example for national and synthetic cumulative distributions of absolute road grade

The KS statistic is incorporated with the sample size of each distribution in KS hypothesis testing to determine if the two distributions can be considered statistically equivalent at a given confidence level. A confidence level of $95 \%$ was used as the default for this analysis.

With a null hypothesis that the two distributions are statistically equivalent, KS hypothesis testing will either reject the null hypothesis (implying that the two datasets have been demonstrated to be statistically different) or fail to reject the null hypothesis (implying that the two datasets have not been demonstrated to be statistically different). 


\section{A.4 Impact of Activity Weighting}

Application of activity weights to the raw national distributions was observed to have relatively low impact on cumulative distributions of absolute road grade and half hill distances as observed in Figures 27 and 28. This result prompted a search for the underlying cause of activityweighting having minimal impacts.

The first theory explored was that distance- and activity-based weights at the county level could be quite similar. To test this theory, distance- and activity-based county weights scatter against one another in Figure A6. A least squares linear fit to the data resulted in an $\mathrm{R}^{2}$ value of 0.6876 . While this shows that there was indeed some correlation between the two sets of weighting terms, we wanted to know more about the counties with large discrepancies between distanceand activity-based weights.

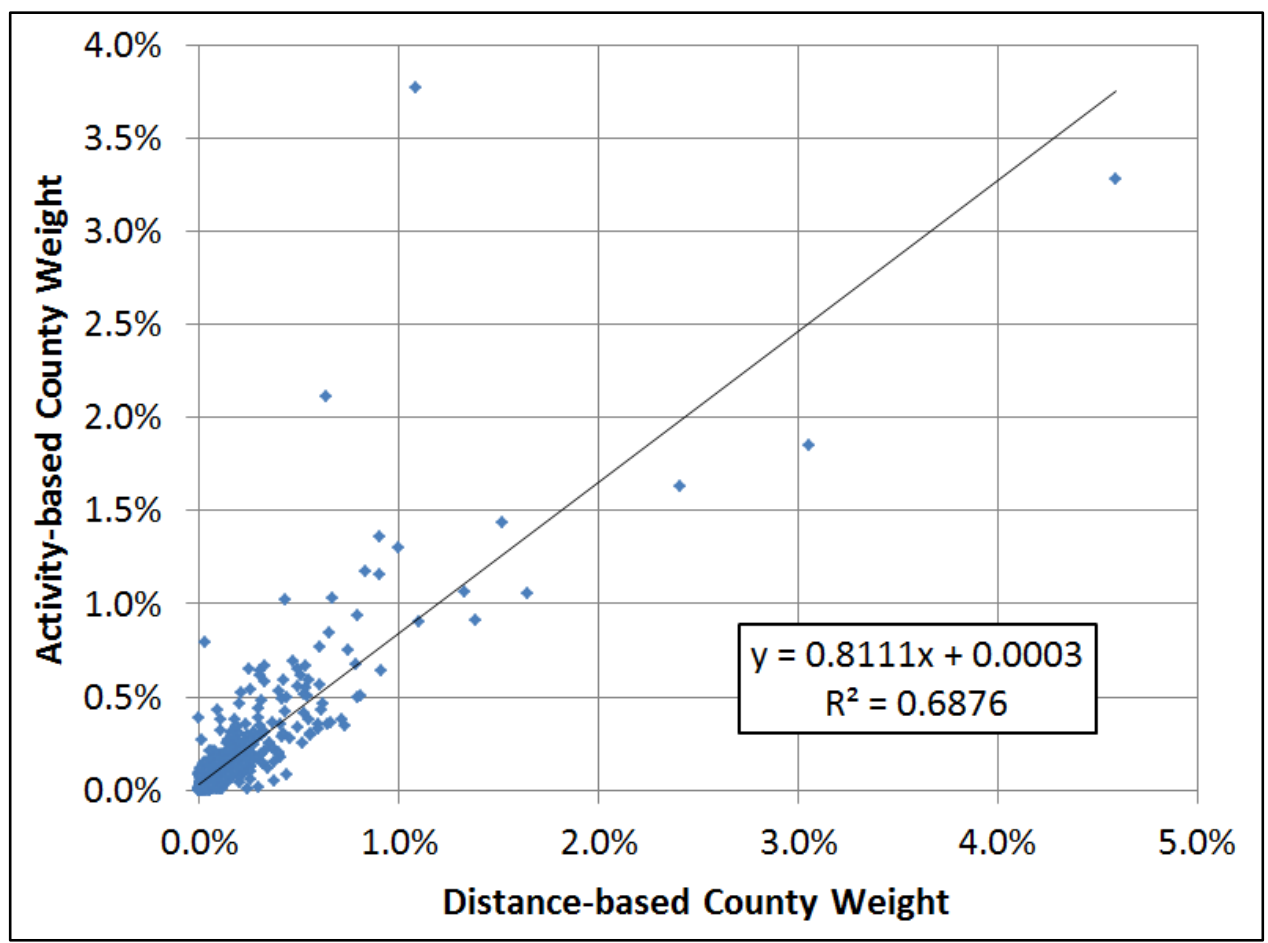

Figure A6. Scatter of activity-based versus distance-based county weights for the sample of 1,728 U.S. counties with controlled access highways

Our next step was to scatter average absolute grade versus the difference between activity- and distance-based weights (as shown in Figure A7). Two solid lines are provided for reference on this plot: 1) the vertical green line indicates counties with identical activity- and distance-based weights, and 2) the horizontal red line indicates the average absolute grade across all counties. 


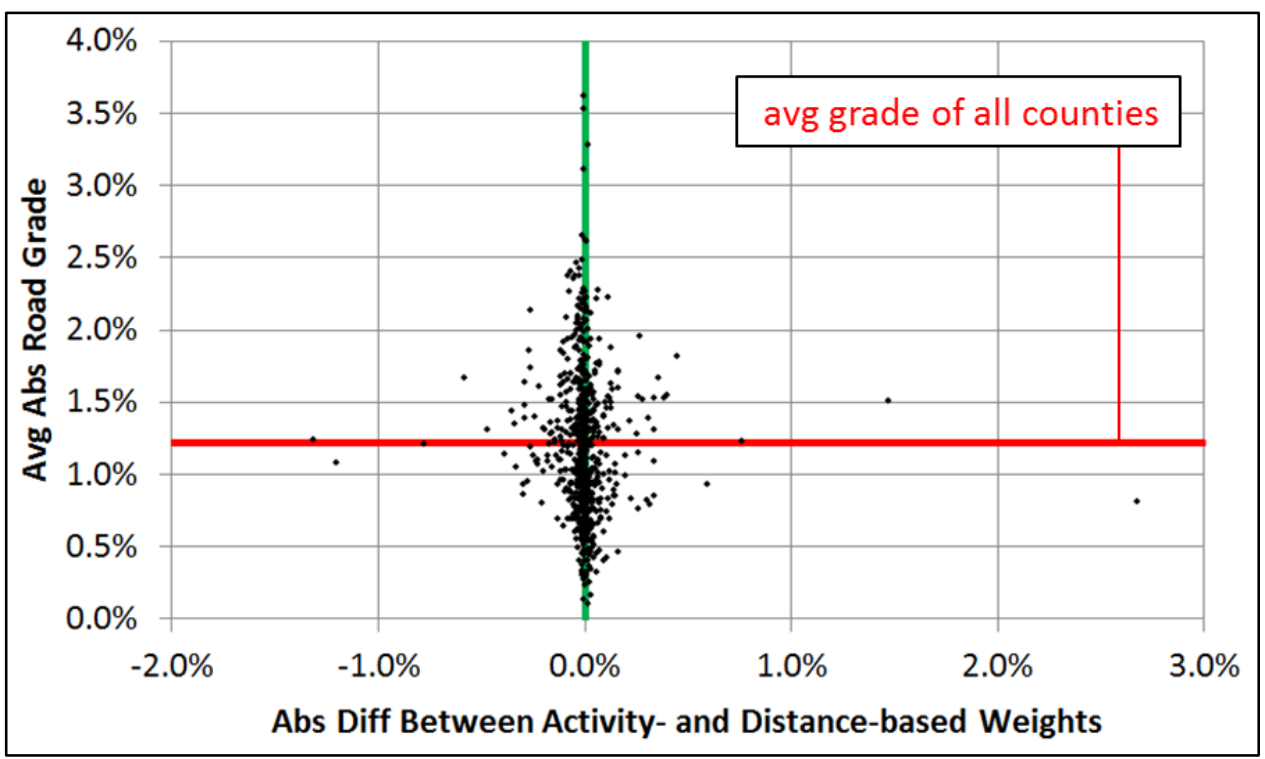

Figure A7. Scatter of average absolute road grade versus the difference between activity- and distance-based weights for the sample of 1,728 U.S. counties with controlled access highways

In order for application of activity weights to significantly impact our national raw distributions, a large number of counties would have need to have significant discrepancies between activityand distance-based weights AND those counties would also have to exhibited statistics deviating from the average county. This analysis shows that while a number of data points met one of these criteria, very few met both. Consequently, the conclusion that application of activity-weighting to our raw distributions had relatively little impact is considered defensible.

\section{A.5 Distributions of Grade by Distance versus Distributions of Average Half Hill Grade}

National road grade distributions were developed by two methods in this analysis:

- Section 3.1, Grade by Distance: Query all U.S. controlled access roads at uniform 52.8foot intervals (100 points per mile) using linear interpolation

- Section 3.2, Average Half Hill Grade: Segment U.S. controlled access highway network into half hill sections and measure distance-averaged grade from each section.

National distributions of road grade by distance introduced in Section 3.1 were selected as the primary road grade metric in this analysis. Activity-weighting was applied to grade by distance distributions in Section 3.3 and used as the target for sample synthetic and local profiles presented in Section 4. By selecting grade by distance distribution as national target metric, this analysis seeks to develop sample grade profiles with proportions of driven distance at each grade bin that are comparable to national estimates for MD/HD trucks.

Aggregation of road grade data into half hill averages results in the loss of information regarding the distribution of grade within each half hill. Even when correcting for half hill distance, half hill aggregations feature a lower degree of grade variability relative to grade by distance distributions. Figure A8 illustrates this point by plotting the national raw distance-based cumulative distributions of grade by distance and average half hill grade for the composite 55- 
mph test group (a plot of the 65-mph test group features similar trends). This plot confirms the expected lack of variability exhibited by the distribution of average half hill grade. The national distribution of grade by distance can be seen to exhibit significantly higher percentages of data below $0.5 \%$ grade and above $2.5 \%$ grade.

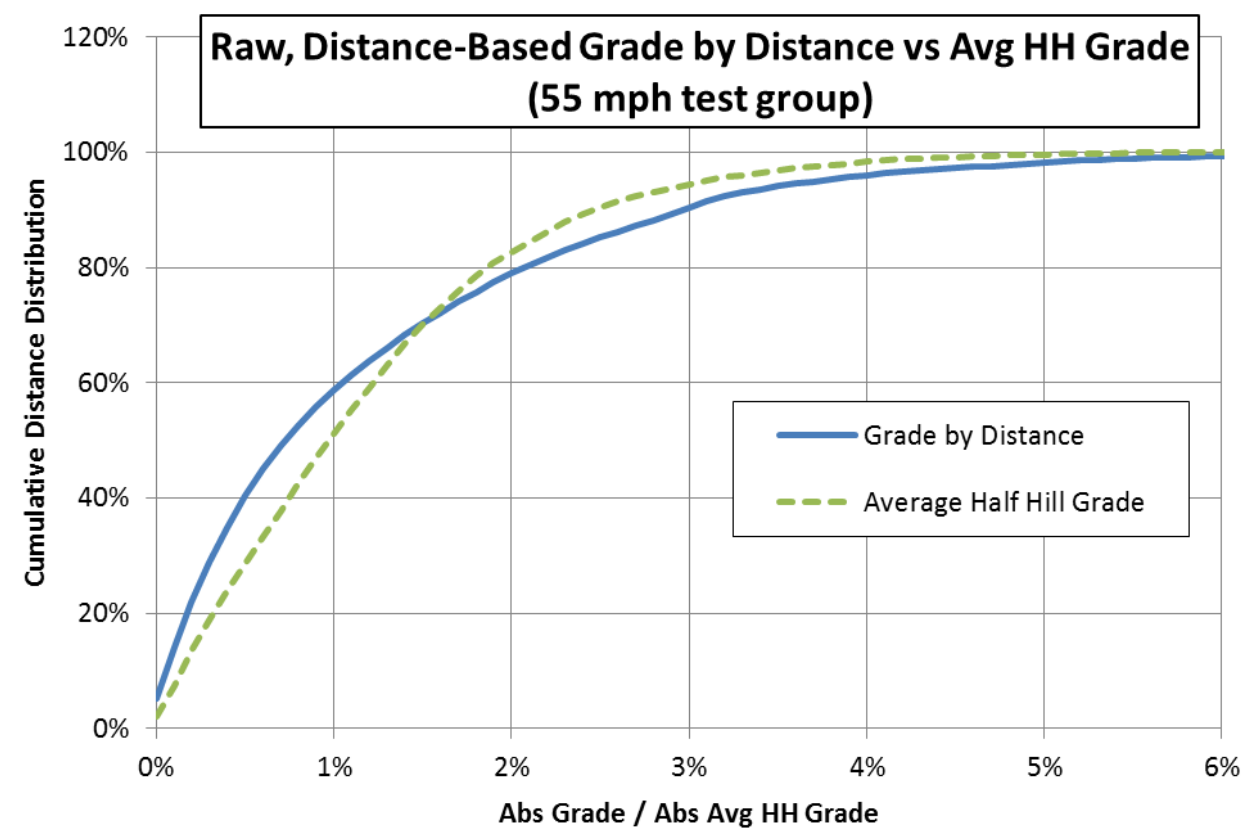

Figure A8. Raw distance-based cumulative distributions of grade by distance and average half hill grade for the 55-mph test group 


\section{Appendix B: Supplementary Distributions from Half Hill Analysis}

Half hill analyses (discussed in Section 3.2) resulted in the generation of multiple data distributions with regard to distance, average grade, and grade ratio (maximum to average). These distributions were segmented by truck speed limit and half hill distance, plotted as frequency- and distance-based distributions, and generated with and without activity weighting. While many of these distributions were not incorporated into the body of the analysis, supplementary distributions are provided in Figures B1 through B9 as points of reference.

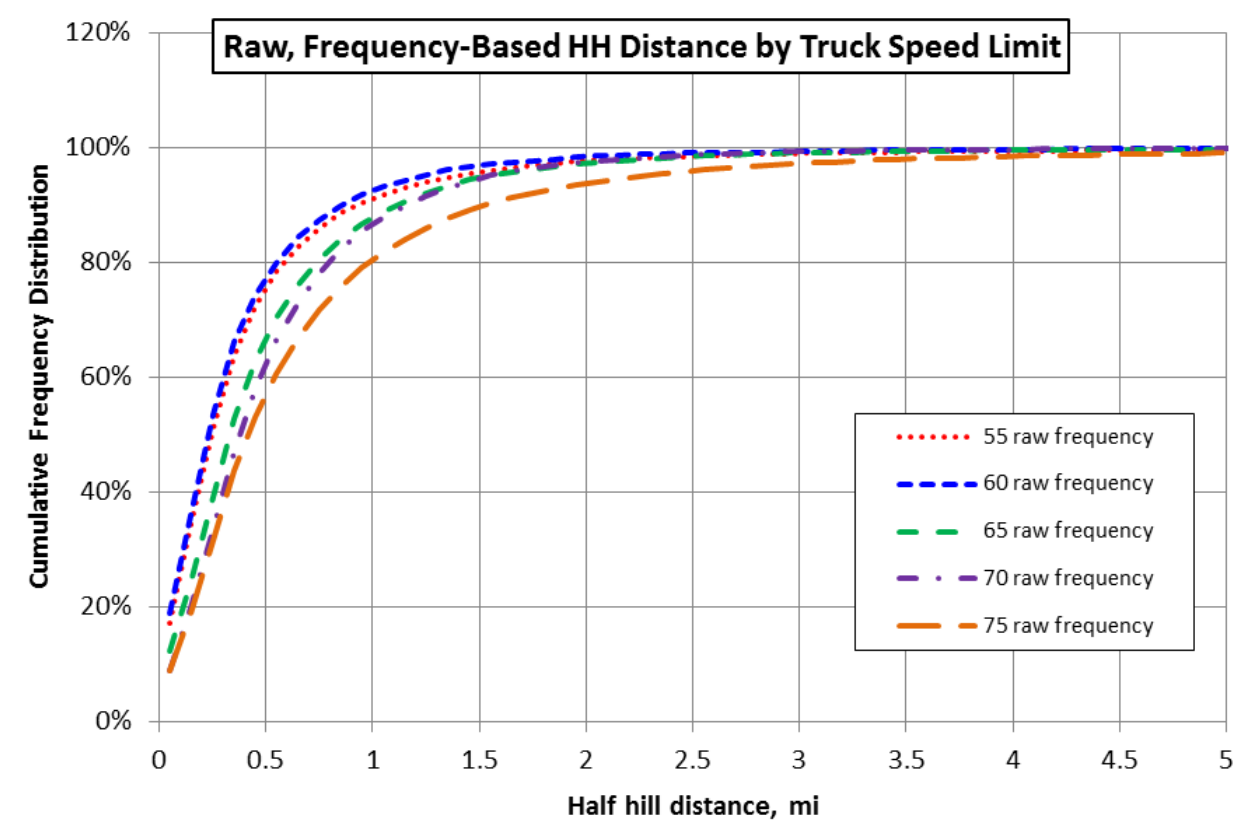

Figure B1. Raw frequency-based cumulative distributions of half hill distance by truck speed limit 


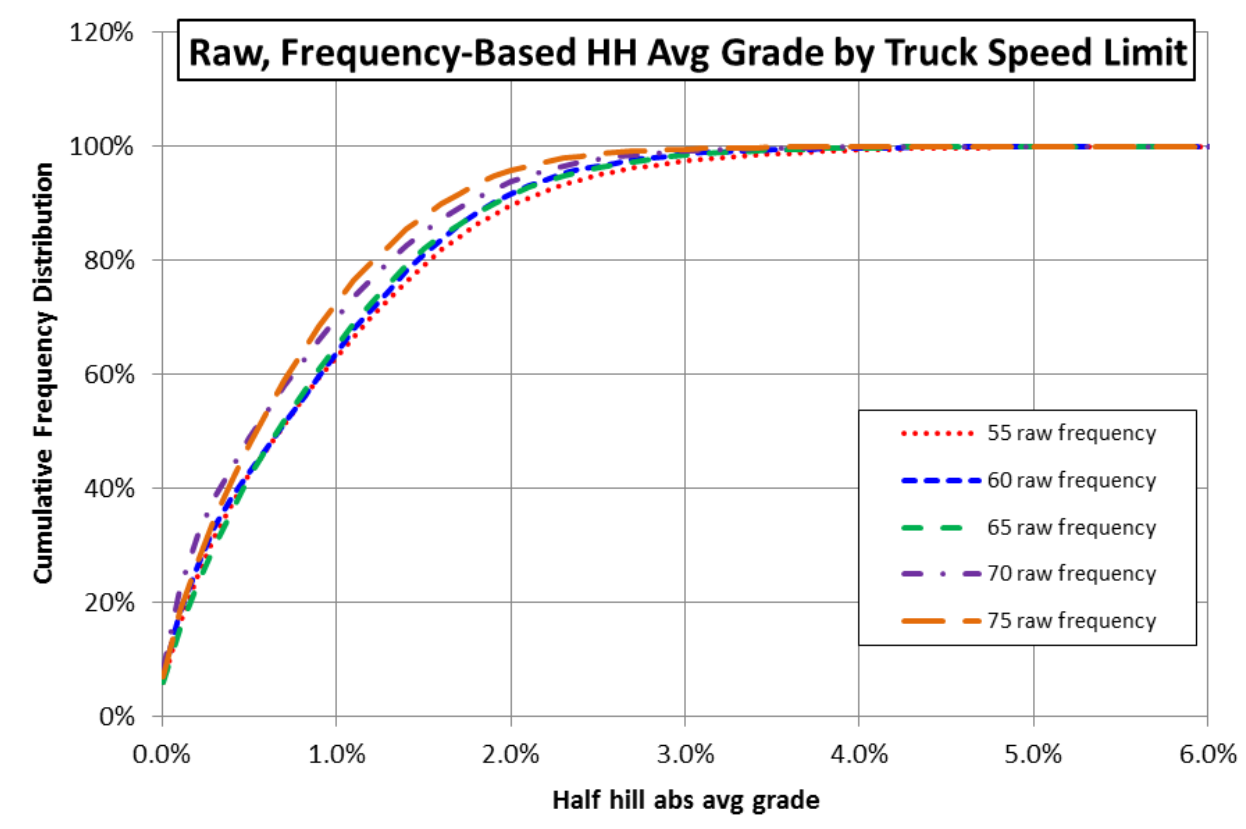

Figure B2. Raw frequency-based cumulative distributions of half hill average grade by truck speed limit

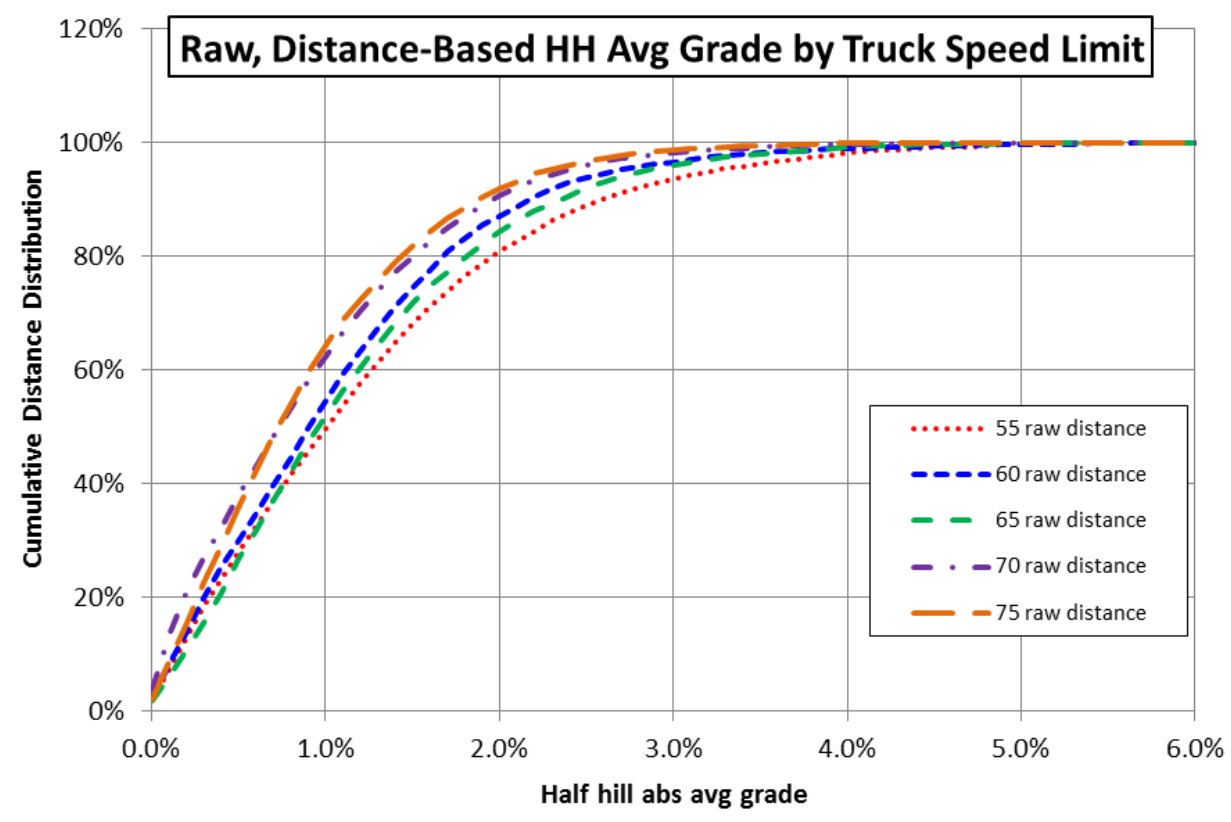

Figure B3. Raw distance-based cumulative distributions of half hill average grade by truck speed limit 


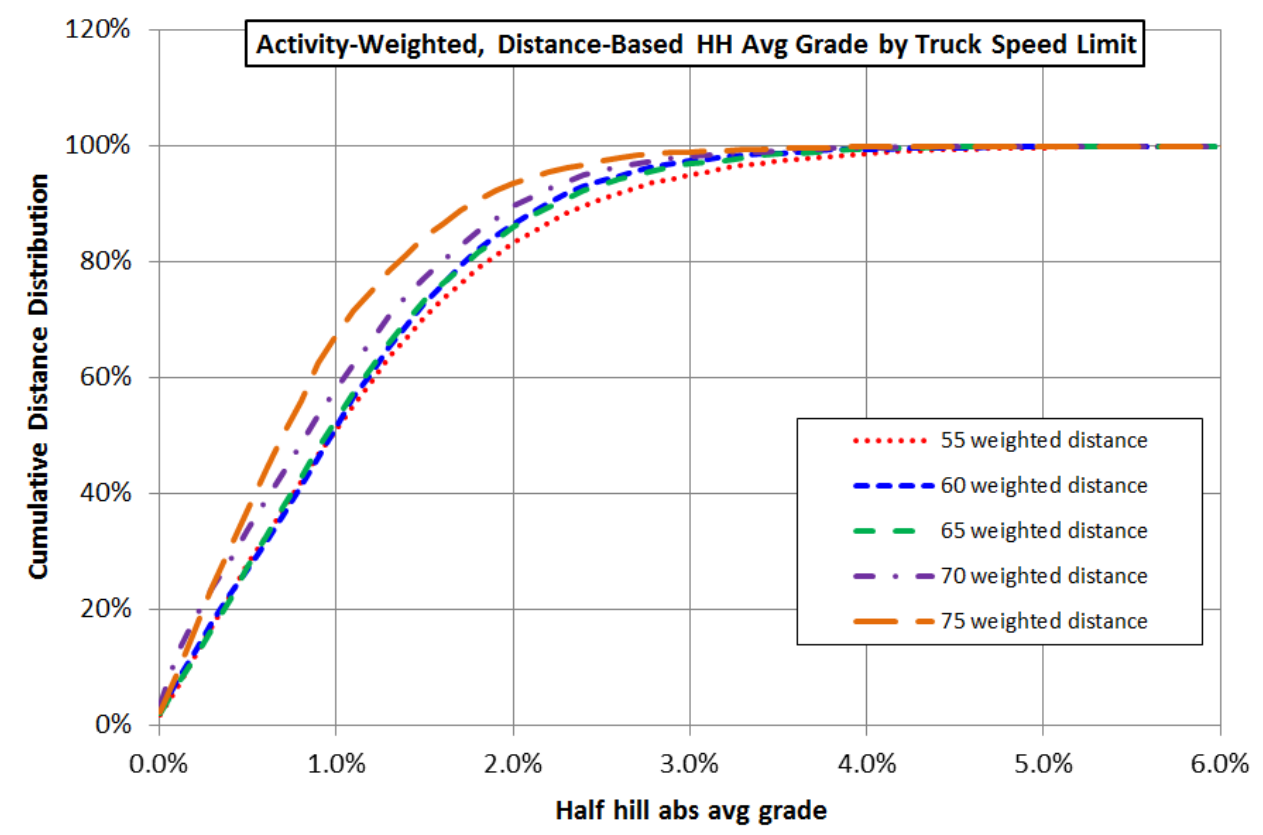

Figure B4. Activity-weighted distance-based cumulative distributions of half hill average grade by truck speed limit

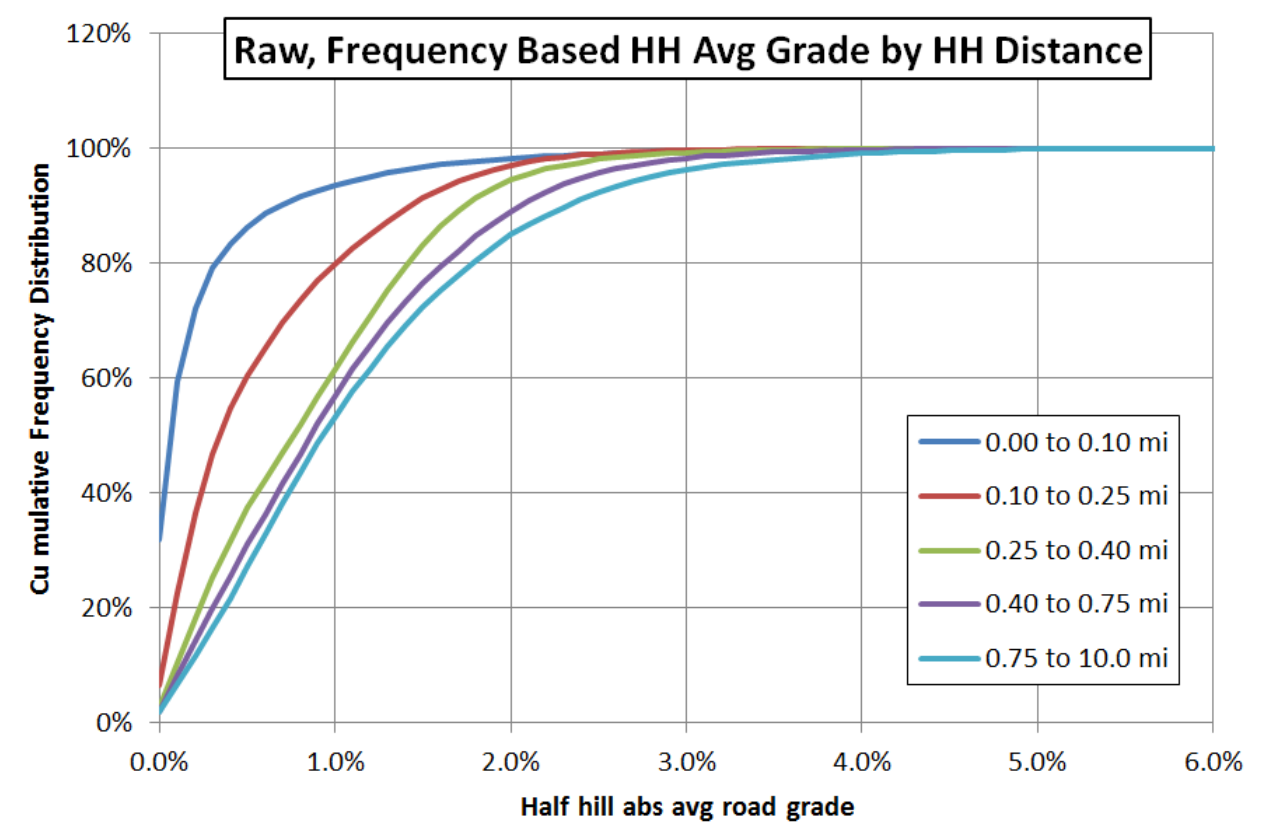

Figure B5. Raw frequency-based cumulative distributions of half hill average grade by half hill distance 


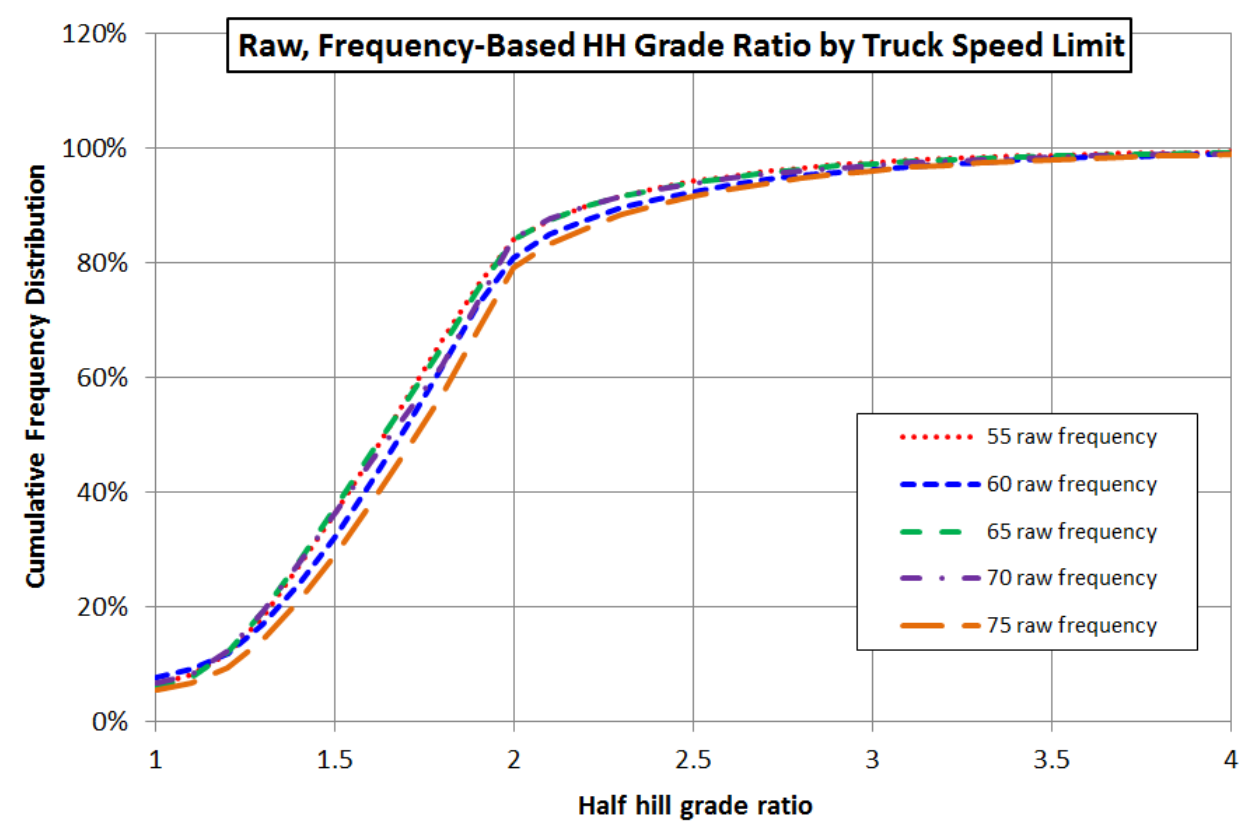

Figure B6. Raw frequency-based cumulative distributions of half hill grade ratio (maximum to average half hill grade) by truck speed limit

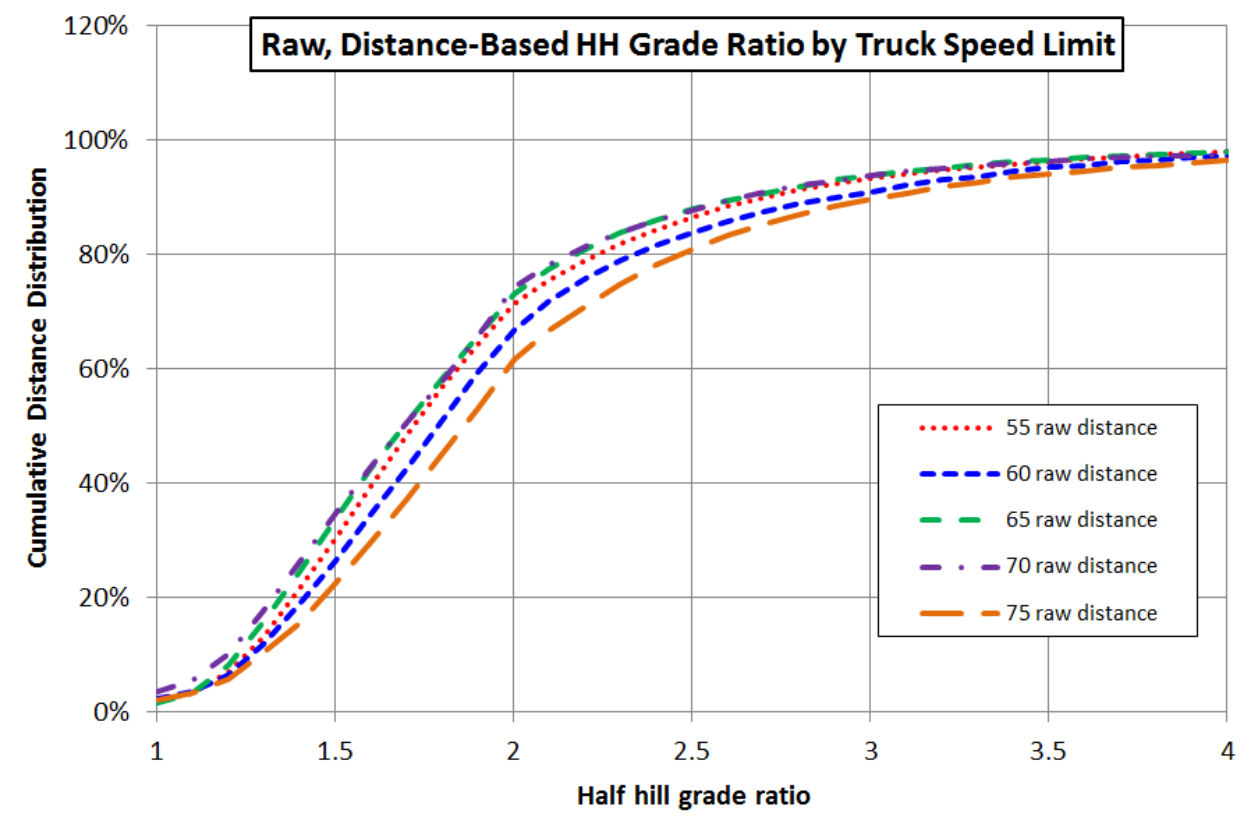

Figure B7. Raw distance-based cumulative distributions of half hill grade ratio (maximum to average half hill grade) by truck speed limit 


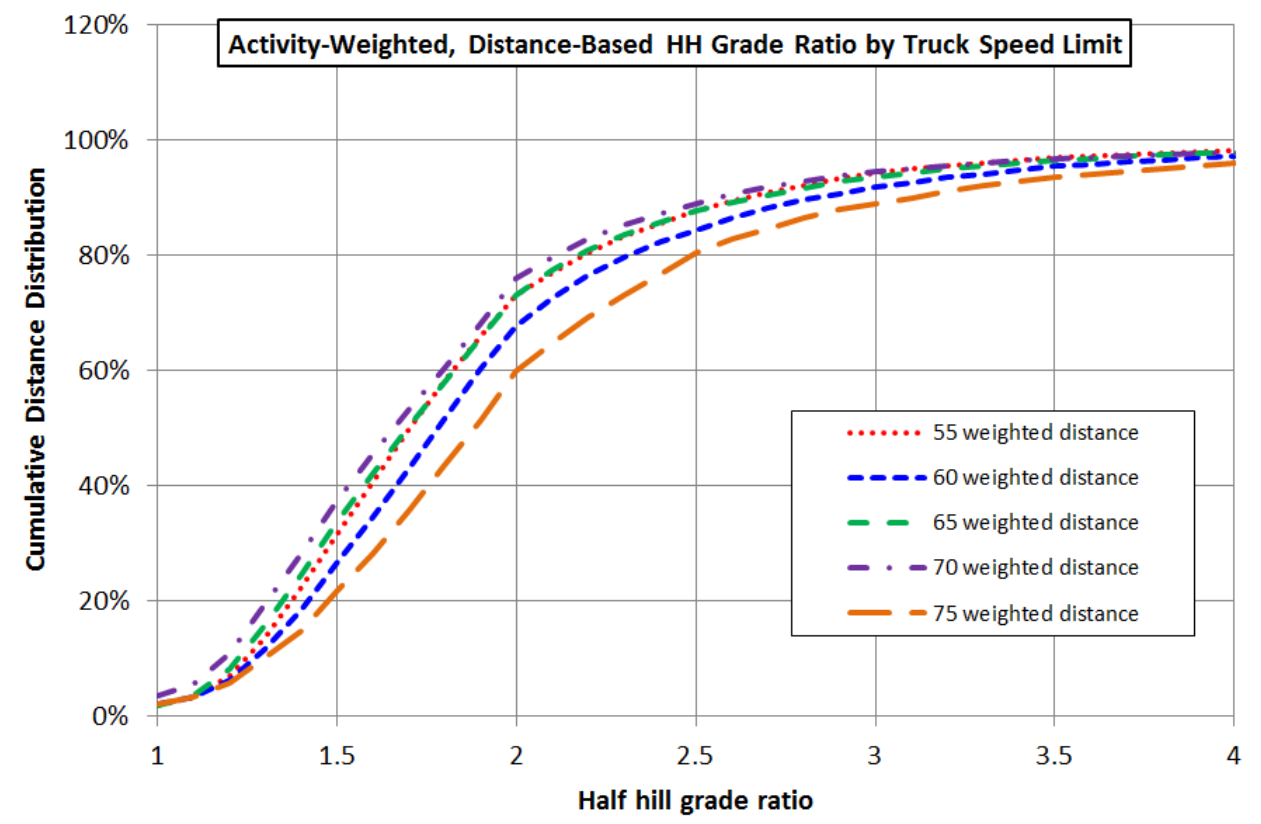

Figure B8. Activity-weighted distance-based cumulative distributions of half hill grade ratio (maximum to average half hill grade) by truck speed limit

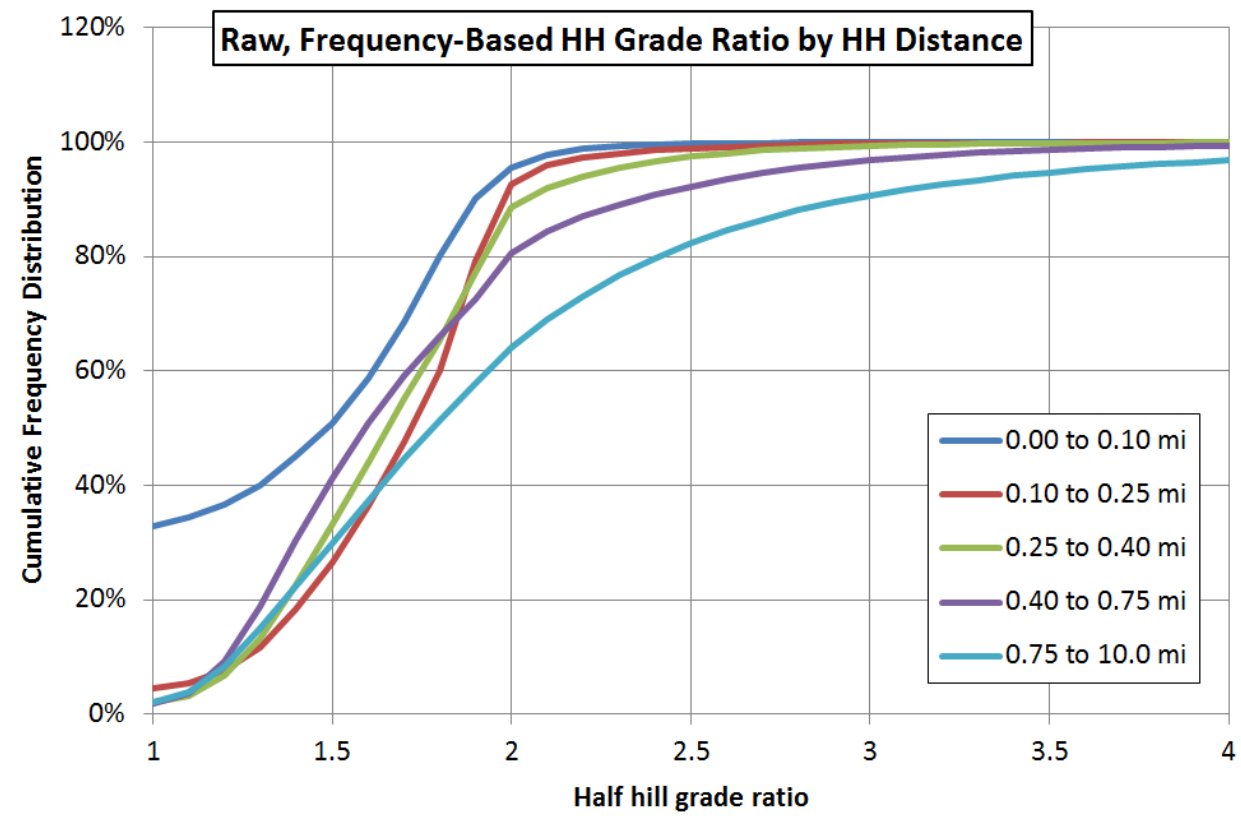

Figure B9. Raw frequency-based cumulative distributions of half hill grade ratio (maximum to average half hill grade) by half hill distance 